\title{
U.S. Hydropower Resource Assessment
}

\author{
for
}

\section{Arkansas}

James E. Francfort

Published December 1993

\section{Idaho National Engineering Laboratory EG\&G Idaho, Inc. Idaho Falls, Idaho 83415}

Prepared for the

U.S. Department of Energy

Assistant Secretary for Energy Efficiency and Renewable Energy Idaho Operations Office

Under DOE Contract DE-AC07-76ID01570 


\begin{abstract}
The Department of Energy is developing an estimate of the hydropower development potential in this country. The Hydropower Evaluation Software (HES) is a computer model that was developed by the Idaho National Engineering Laboratory for this purpose. The HES measures the potential hydropower resources available in the United States, using uniform criteria for measure' tent. The software was developed and tested using hydropower information and data provided by the Southwestern Power Administration. It is a dBASE menu-driven software application that allows the personal computer user to assign environmental attributes to potential hydropower sites, calculate development suitability factors for each site based on the environmental attributes present, and generate reports based on these suitability factors. This report details the resource assessment results for the state of Arkansas.
\end{abstract}




\section{CONTENTS}

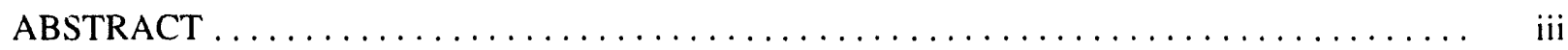

INTRODUCTION $\ldots \ldots \ldots \ldots \ldots \ldots \ldots \ldots \ldots \ldots \ldots \ldots \ldots \ldots \ldots \ldots \ldots \ldots \ldots \ldots$

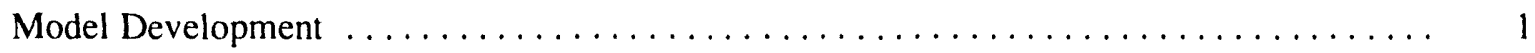

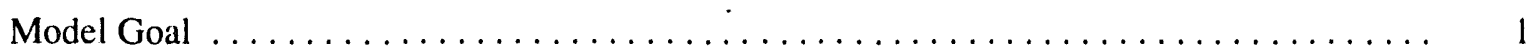

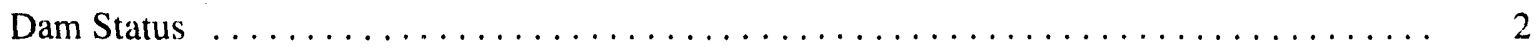

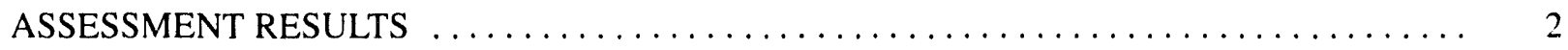

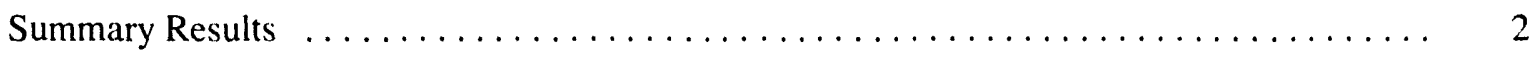

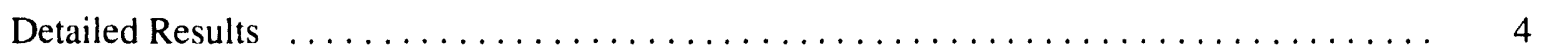

OBTAINING INDIVIDUAL STATE INFORMATION $\ldots \ldots \ldots \ldots \ldots \ldots \ldots \ldots \ldots \ldots$

ADDITIONAL HYDROPOWER EVALUATION SOFTWARE INFORMATION $\ldots \ldots \ldots \ldots$

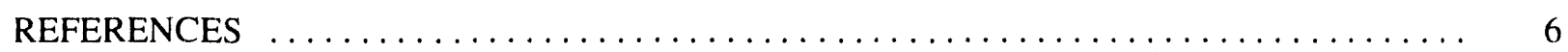

Appendix A-Hydropower Capacity Summary $\ldots \ldots \ldots \ldots \ldots \ldots \ldots \ldots \ldots \ldots \ldots \ldots \ldots \ldots$

Appendix B-Hydropower Resource Assessment by River Basin $\ldots \ldots \ldots \ldots \ldots \ldots \ldots \ldots$ B-1

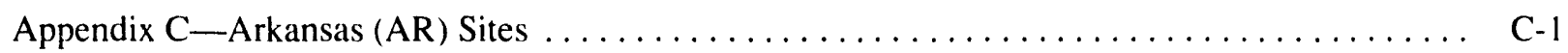

Appendix D-Resource Database Listing $\ldots \ldots \ldots \ldots \ldots \ldots \ldots \ldots \ldots \ldots \ldots \ldots \ldots \ldots \ldots \ldots \ldots \ldots$

\section{FIGURES}

1. Number of sites with various capacity potentials $\ldots \ldots \ldots \ldots \ldots \ldots \ldots \ldots \ldots \ldots$

2. The Hydropower Evaluation Software identified potential hydropower capacity and the unadjusted potential capacity

3. The number of sites with potential hydropower capacity and the total megawatts of potential capacity

4. Number of sites with potential hydropower capacity in the Arkansas river basins . . . . . . 4

5. Potential hydropower capacity in the Arkansas river basins $\ldots \ldots \ldots \ldots \ldots \ldots \ldots \ldots$ 


\section{U.S. Hydropower Resource Assessment for Arkansas}

\section{INTRODUCTION}

In June 1989, the U.S. Department of Energy initiated the development of a National Energy Strategy to identify the energy resources available to support the expanding demand for energy in the United States. Public hearings conducted as part of the strategy development process indicated that potential hydropower resources were not well defined. As a result, the Department of Energy established an interagency Hydropower Resource Assessment Team to ascertain the hydropower potential. In connection with these efforts by the Department of Energy, the Idaho National Engineering Laboratory designed the Hydropower Evaluation Software (HES), which has been used to perform a resource assessment of the undeveloped hydropower potential in Arkansas (as well as several other states). This report presents the results of the hydropower resource assessment for the state of Arkansas. Pumped storage hydropower potential is not included.

The HES was developed as a tool to measure hydropower potential by regional power marketing administrations and state energy agencies, because they were the most likely to have and need accurate hydropower information. The HES was not intended to provide precise development factors for individual sites, but to provide regional or state capacity totals. Because the software was developed as a generic measurement tool encompassing national issues, regional and state totals must be considered judiciously; various local issues may skew hydropower potential totals. The information for the resource assessment was compiled from the Federal Energy Regulatory Commission's Hydropower Resource Assessment database and several other sources. Refer to DOE/ID-10338, the User's Manual (Francfort, Matthews, Rinehart, 1991) for the specifics of the software and to DOE/ID-10430, the Status Report (Francfort, Moore,
Rinehart, 1993) for an overview of all resource assessment activities to date.

\section{Model Development}

Hydropower Evaluation Software, both a probability-factor computer model and a data base, is a dBASE, menu-driven software application that is intended to be user-friendly. Computer screens and report generation capabilities were developed to meet the needs of users nationwide. The software uses environmental attribute data to generate an overall project environmental suitability factor (PESF) between 0.1 and 0.9 , where 0.9 indicates the highest likelihood of development and 0.1 indicates the lowest likelihood of development. Suitability factors depend on environmental attributes of a potential site. They reflect the considerations that (a) environmental concerns can make a potential site unacceptable. prohibiting its development (for a suitability factor of 0.1 ), or (b) absence of environmental concern can have little or no effect on the likelihood of site development (for a suitability factor of $0.9)$. A combination of attributes results in a lower suitability factor because multiple environmental considerations reduce the likelihood that a site may be developed to its physical potential.

\section{Model Goal}

The goal of the HES is to assemble an accurate resource database of all potential hydropower sites in the United States for use as a planning tool to determine the viable national hydropower potential. Potential hydropower is not limited to the development of new sites; it also includes the development of additional hydropower at sites that currently have hydropower but are not developed to their full potential. This hydropower potential is a source of nonpolluting, renewable energy available to meet the growing power needs of the United States. The HES should make 
this goal attainable and help assure a set of uniform criteria for national assessment.

\section{Dam Status}

The effects of environmental attributes vary by dam status. The dam status classifications follow the Federal Energy Regulatory Commission (FERC) standard, which is

$$
\begin{aligned}
& \mathrm{W}= \text { Developed hydropower site with } \\
& \text { power. The total hydropower } \\
& \text { capacity has not been fully } \\
& \text { developed. Only the undevel- } \\
& \text { oped capacity is discussed in this } \\
& \text { report. }
\end{aligned}
$$

$\mathrm{W} / \mathrm{O}=$ Developed site without power generation. The site has some type of developed impoundment or diversion structure but no hydropower generating capacity.

$\mathrm{U}=$ Undeveloped site. The site does not have power generation capability nor a developed impoundment or diversion structure.

\section{ASSESSMENT RESULTS}

\section{Summary Results}

A total of 61 sites have been identified and assessed for their hydropower potential. Information as to the potential megawatts of capacity for 11 of the sites was not available; however, these sites have been identified as having hydropower potential and are included in the group of 61 . The Hydropower Evaluation Software results for site capacities range from 125 kilowatts to 108 megawatts. Most of the sites have potential capacities of under 10 megawatts (Figure 1).

The unadjusted hydropower potential for Arkansas was identified as being 1,209 megawatts. The Hydropower Evaluation Software results lower this estimate 39\% to 737 megawatts. The greatest reduction in undeveloped potential occurs at sites with no physical structures present. These undeveloped sites have a Hydropower Evaluation Software estimated capacity of 231 megawatts, a $64 \%$ reduction in capacity (Figure 2). The number of sites does not change, only the identified capacity is reassessed (Figure 3 ).

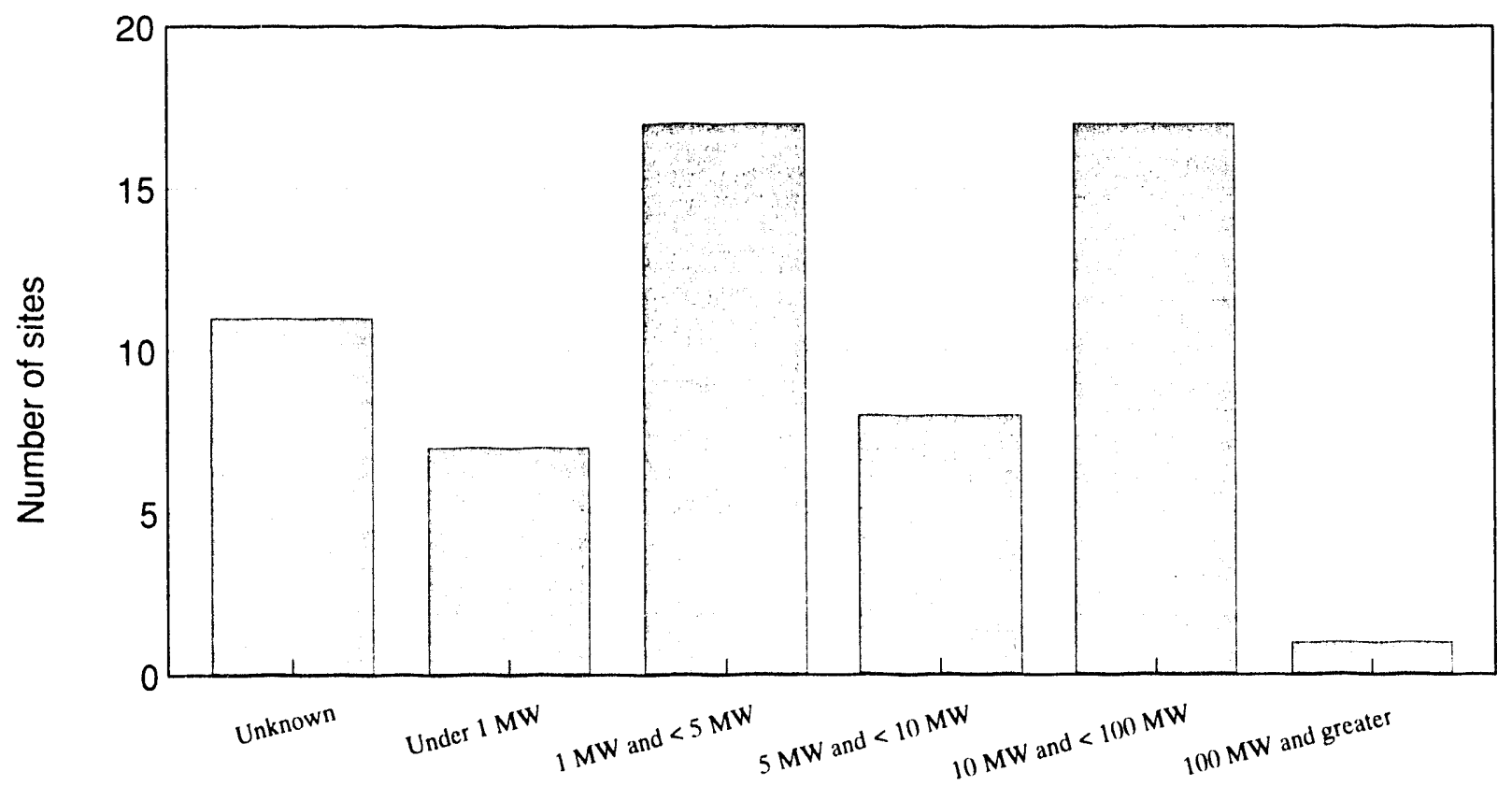

Figure 1. Number of sites with various capacity potentials. The unknown category is for sites that have hydropower potential but the size of the potential is undefined. 


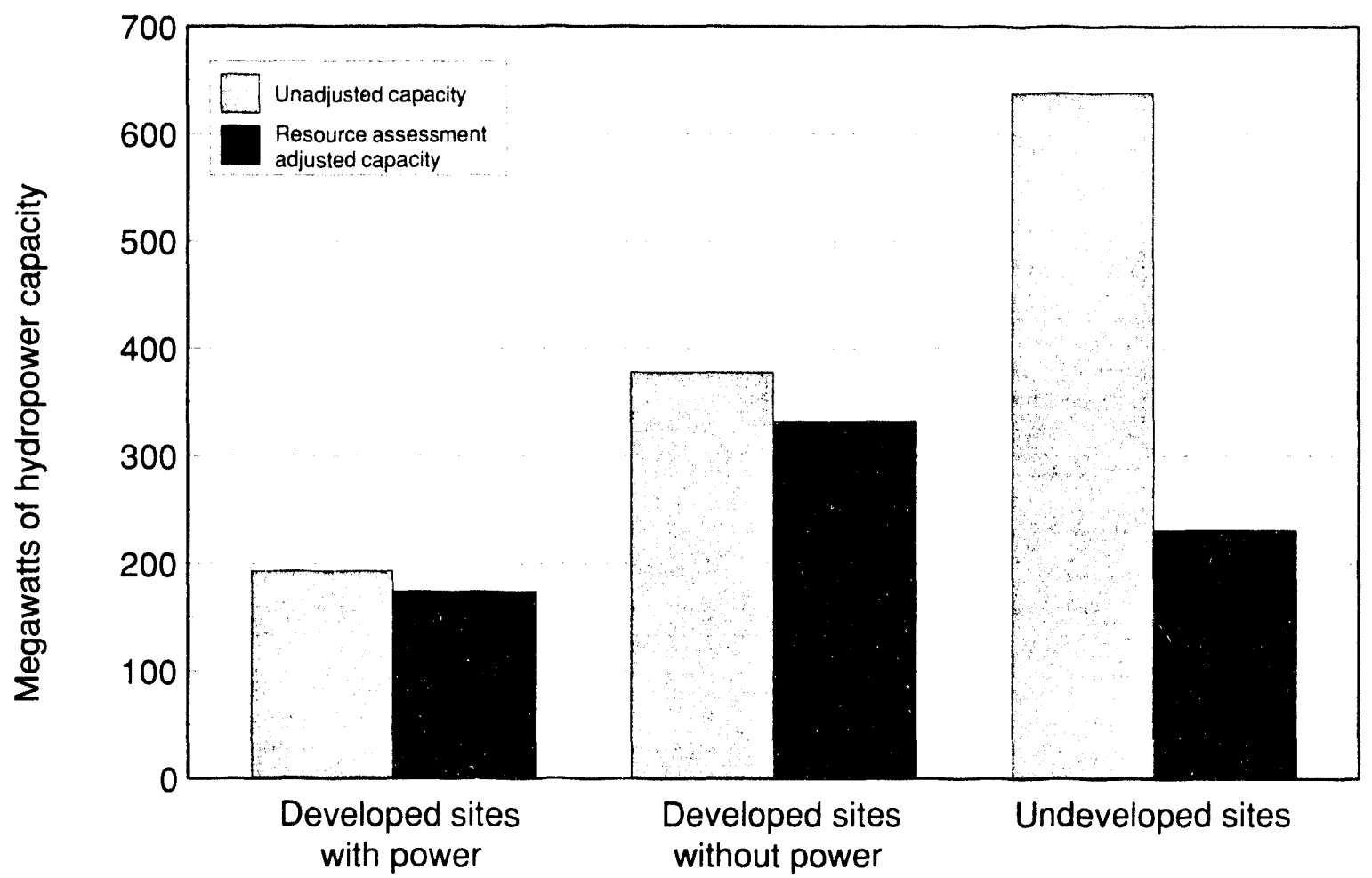

Figure 2. The Hydropower Evaluation Software identified potential hydropower capacity and the unadjusted potential capacity.

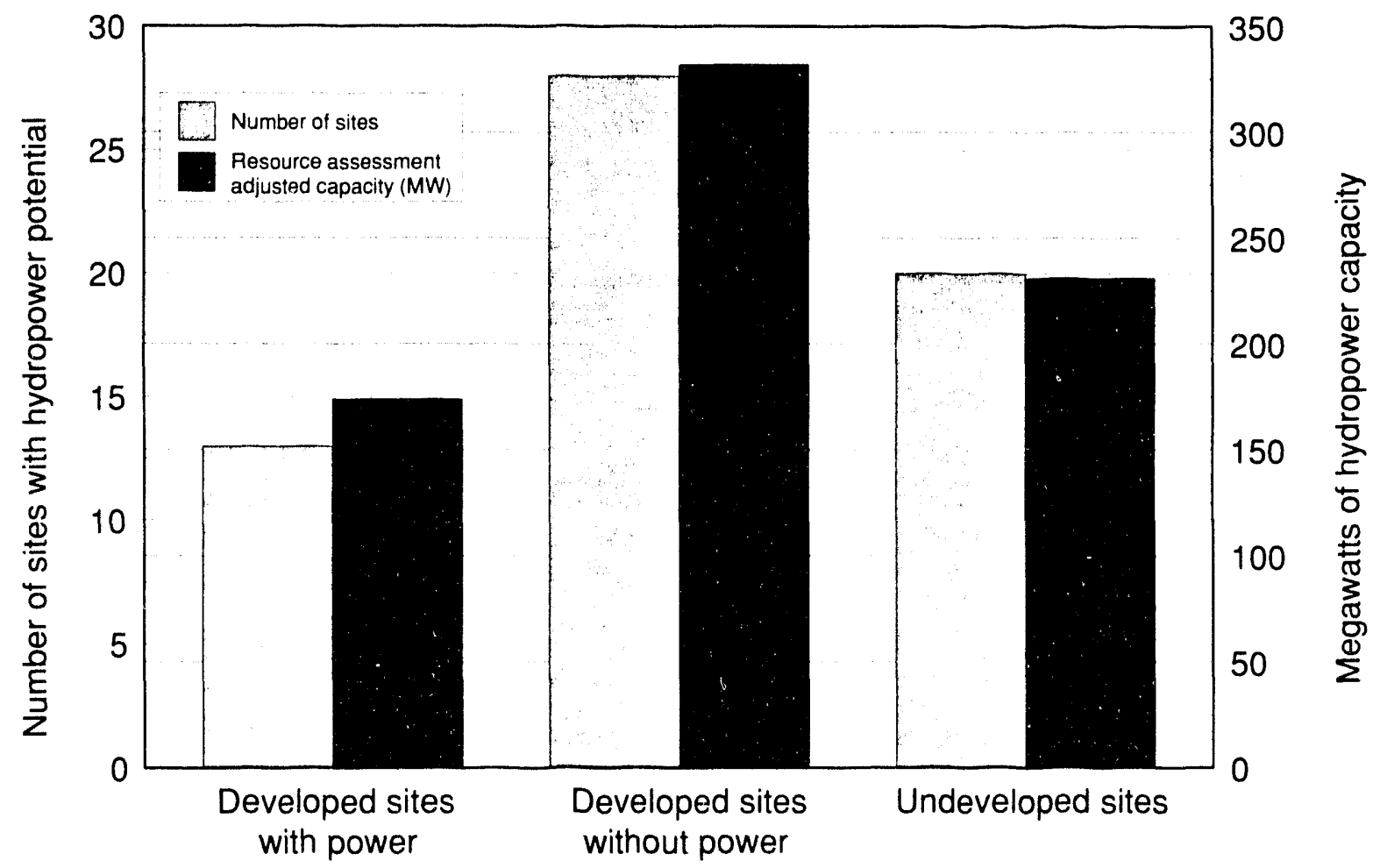

Figure 3. The number of sites with potential hydropower capacity and the total megawatts of potential capacity. 
The 61 identified sites are located within 5 river basins. The number of sites per river basin ranges from 7 each in both the Lower Red River Basin and the Upper White River Basin to 18 sites in the Lower White River Basin (Figure 4). The Lower Arkansas River Basin has the most undeveloped potential hydropower capacity of the Arkansas river basins (Figure 5).

\section{Detailed Results}

The appendices contain, in the form of Hydropower Evaluation Software generated reports, detailed information of the potential hydropower capacity in Arkansas. The appendices contain the following information:

Appendix A The hydropower capacity summary for Arkansas categorizes sites by dam status. The number of sites, unadjusted capacity, and Hydropower Evaluation Software adjusted capacity are provided based on the dam status.

Appendix B The hydropower resource assessment by river basin includes the FERC project number, project name, stream name, dam status, unadjusted capacity, and Hydropower Evaluation Software adjusted capacity for each of the individual sites. Subtotals are provided for each river basin.

Appendix C This is a listing of the project numbers, plant name, stream name, if a site is Federally owned, unadjusted capacity, and Hydropower Evaluation Software adjusted capacity. The sites are grouped by dam status.

Appendix D This section contains a resource database listing for each of the 61 sites in Arkansas. Information includes plant, stream, state, county, river basin and owner names; project number; name plate and Hydropower Evaluation Software adjusted capacity ratings; the unit and plant types, dam status; latitude; longitude; and the environmental factors that the Hydropower Evaluation Software uses to determine the project environmental suitability factor.

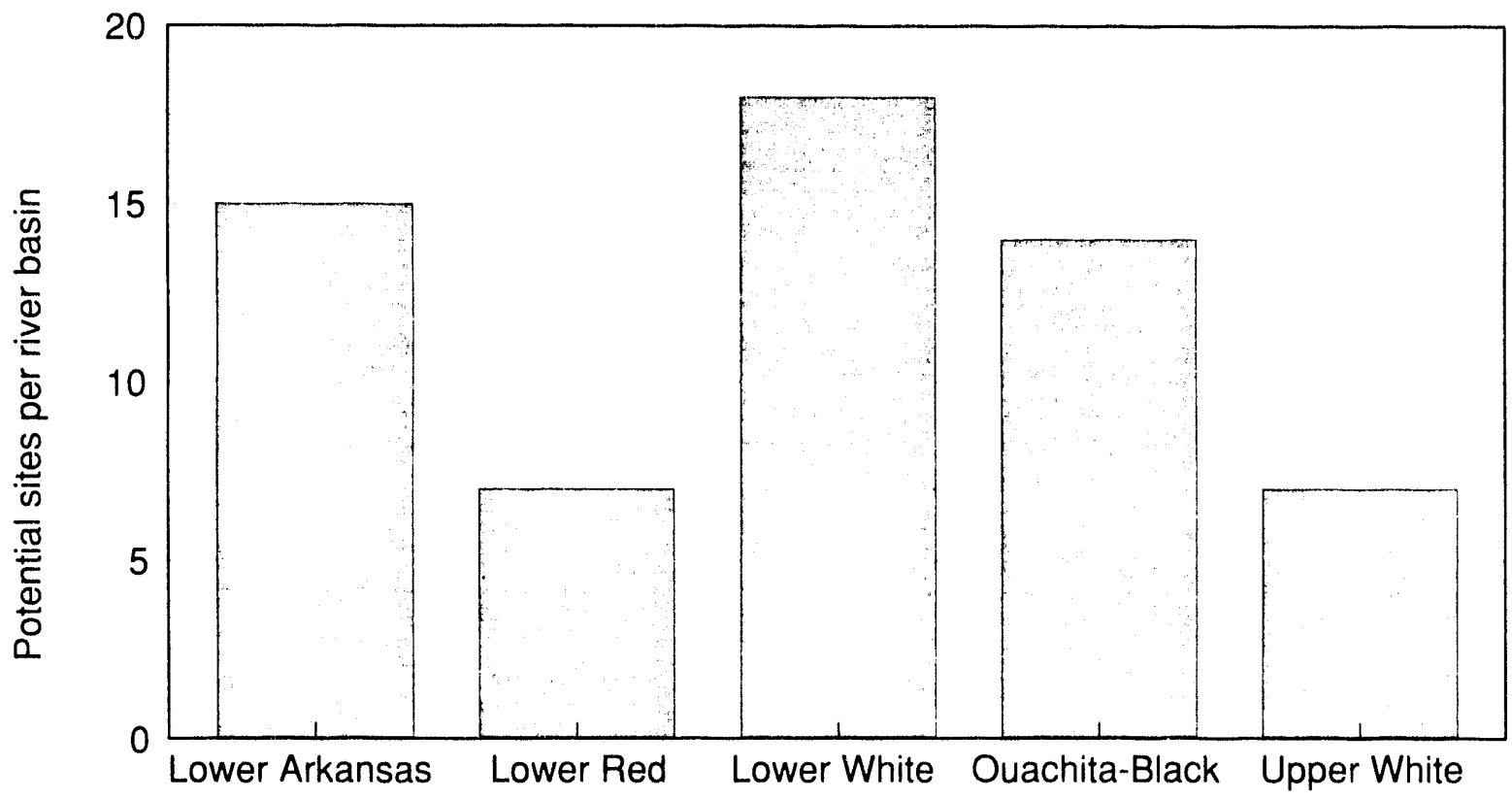

Figure 4. Number of sites with potential hydropower capacity in the Arkansas river basins. 


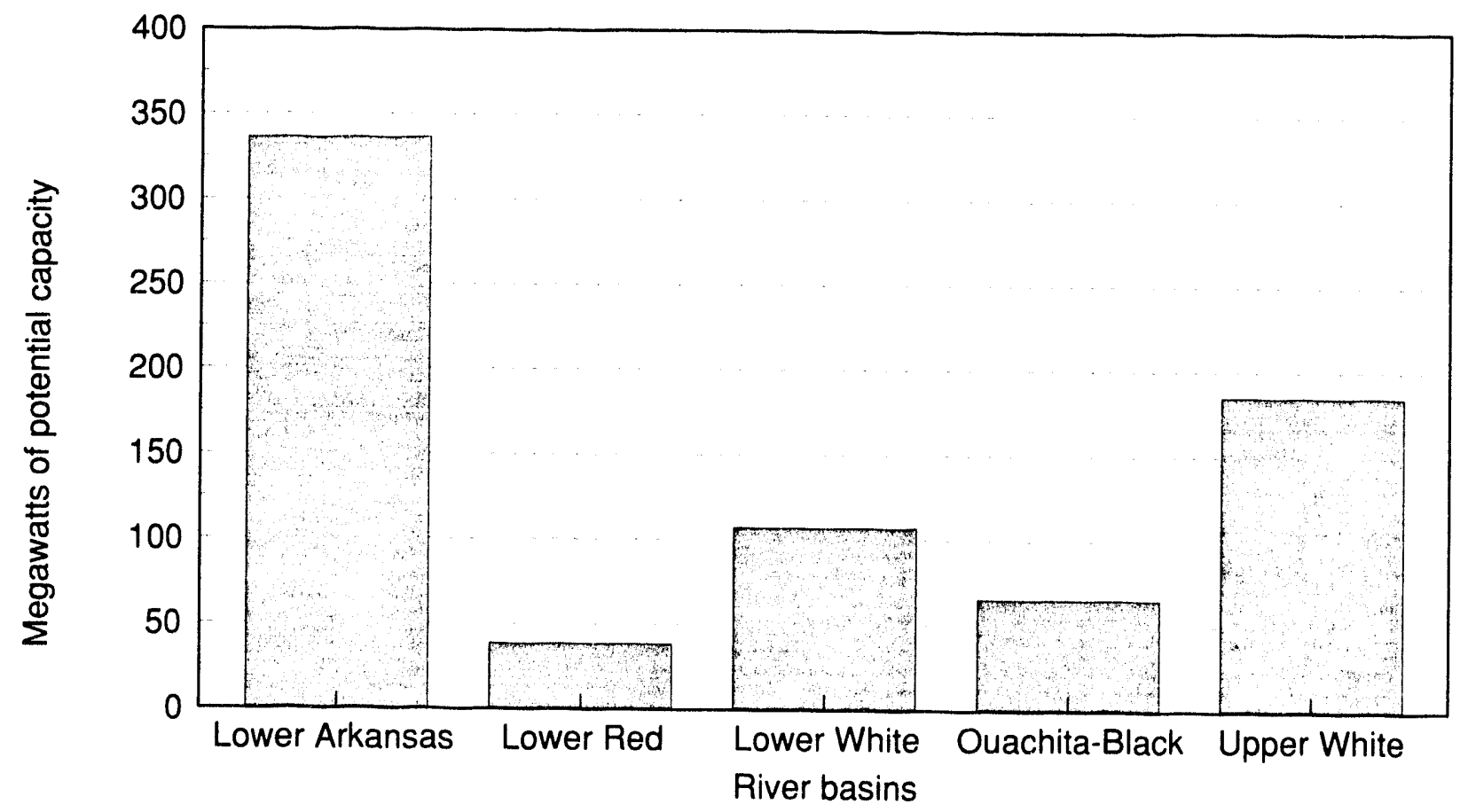

Figure 5. Potential hydropower capacity in the Arkansas river basins.

\section{OBTAINING INDIVIDUAL STATE INFORMATION}

Additional copies of the hydropower resource assessment results for individual states are available and can be obtained by writing or calling the National Technical Information Service (NTIS).

Telephone Orders - (703) 487-4650. NTIS sales desk and customer services are available between 8:30 a.m. and 5:00 p.m., Eastern Standard Time.

Fax - (703) 321-8547. Customers may fax their orders to NTIS. These orders may be charged to a NTIS deposit account, American Express, VISA, or MasterCard.

Mail Orders - Mail orders should be sent to National Technical Information Service, Document Sales, 5285 Port Royal Road, Springfield, VA 22161. Call the sales desk for prices before placing an order.
Method of Payment - Customers may pay for reports (and other NTIS products and services) by (a) credit card (American Express, Visa, or MasterCard); (b) check or money order on a United States bank payable to NTIS; (c) a NTIS deposit account; or (d) by asking to be billed (add $\$ 7.50$ per order, United States, Canada, and Mexico, only).

Handling Fee - A $\$ 3.00$ handling fee per total order applies to orders from the United States, Canada, and Mexico. Handling charges do not apply to rush order service or pick-up orders.

Postage and Shipping - Orders are shipped first class mail, or equivalent, to addresses in the United States, Canada, and Mexico.

Order Turnaround Time - Orders for technical reports generally are shipped within 2 to 8 days of receipt. For faster service, NTIS offers rush order service.

Rush Order Service - Call 1-800-533-NTIS. In Virginia, Canada, and Mexico call 
(703) 487-4700. For NTIS rush order service add $\$ 15.00$ per item. This guarantees that an order will be processed through NTIS within 24 hours of its receipt. These orders receive immediate, individual attention. The items ordered are delivered by first call mail. Call NTIS for information on rush order service for computer products.

For Help in Tracing an Order - Call (703) 487-4650 and request the customer service option.

\section{ADDITIONAL HYDROPOWER EVALUATION SOFTWARE INFORMATION}

Additional information concerning the Hydropower Evaluation Software can be obtained by contacting Ben Rinehart or Jim Francfort at the addresses provided below. Copies of the software and the User's Manual may also be obtained from these individuals.

\author{
Ben Rinehart \\ Idaho National Engineering Laboratory \\ P.O. Box 1625 \\ Idaho Falls, ID 83415-3830 \\ (208) 526-1002 \\ Jim Francfort \\ Idaho National Engineering Laboratory \\ P.O. Box 1625 \\ Idaho Falls, ID 83415-3875 \\ (208) 526-6787
}

Information concerning the state of Arkansas involvement with the resource assessment or about the identified sites may be obtained by contacting:

Earl Smith

Arkansas Soil and Water Conservation

Commission

Suite 2D

1 Capital Mall

Little Rock, AR 72201

(501) 682-1611

\section{REFERENCES}

Francfort, J. E., S. D. Matthews, and B. N. Rinehart, 1991, Hydropower Evaluation Software User's Manual, DOE/ID-10338, Idaho National Engineering Laboratory, Idaho Falls, Idaho.

Francfort, J. E., K. M. Moore, and B. N. Rinehart, 1993, Uniform Criteria for U.S. Hydropower Resource Assessment, Hydropower Evaluation Software Status Report, DOE/ID-10430, Idaho National Engineering Laboratory, Idaho Falls, Idaho. 


\section{Appendix A}

Hydropower Capacity Summary 


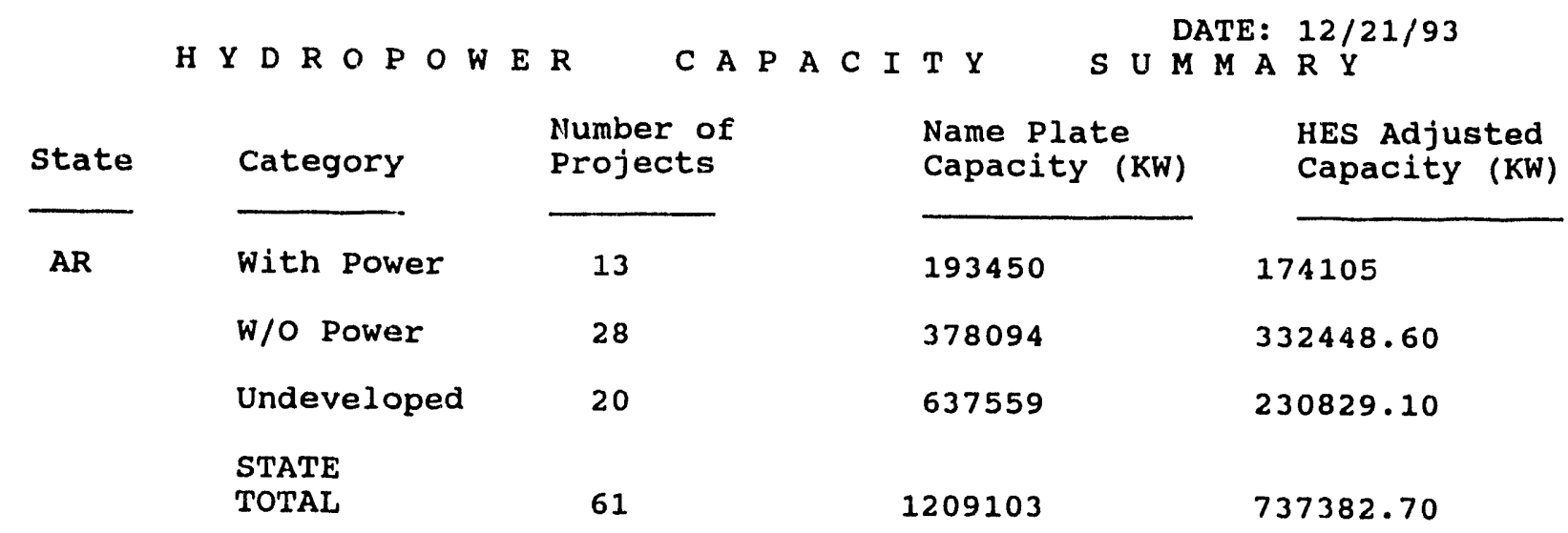




\section{Appendix B}

Hydropower Resource Assessment by River Basin

B-1 


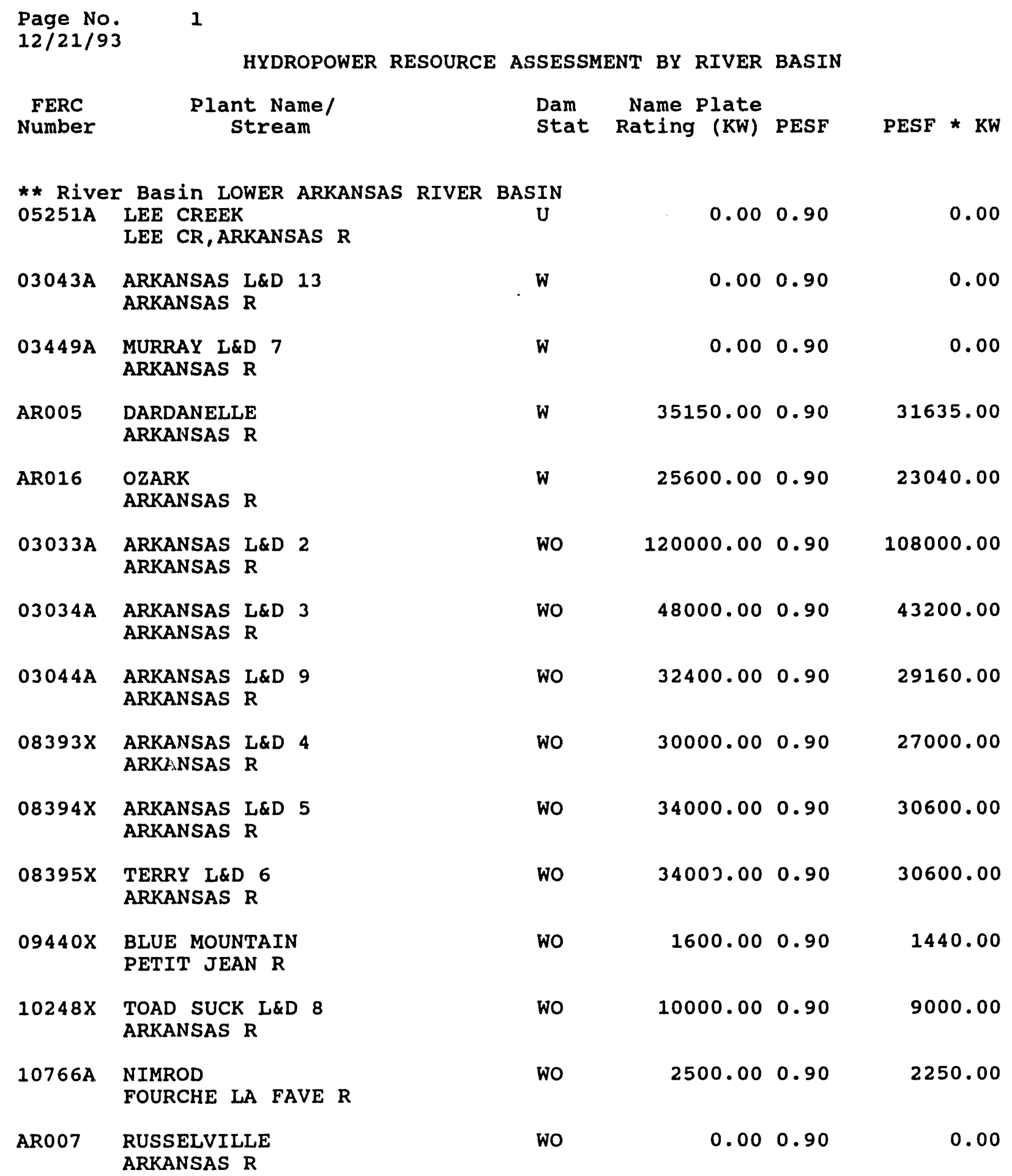


Page No.

$12 / 21 / 93$

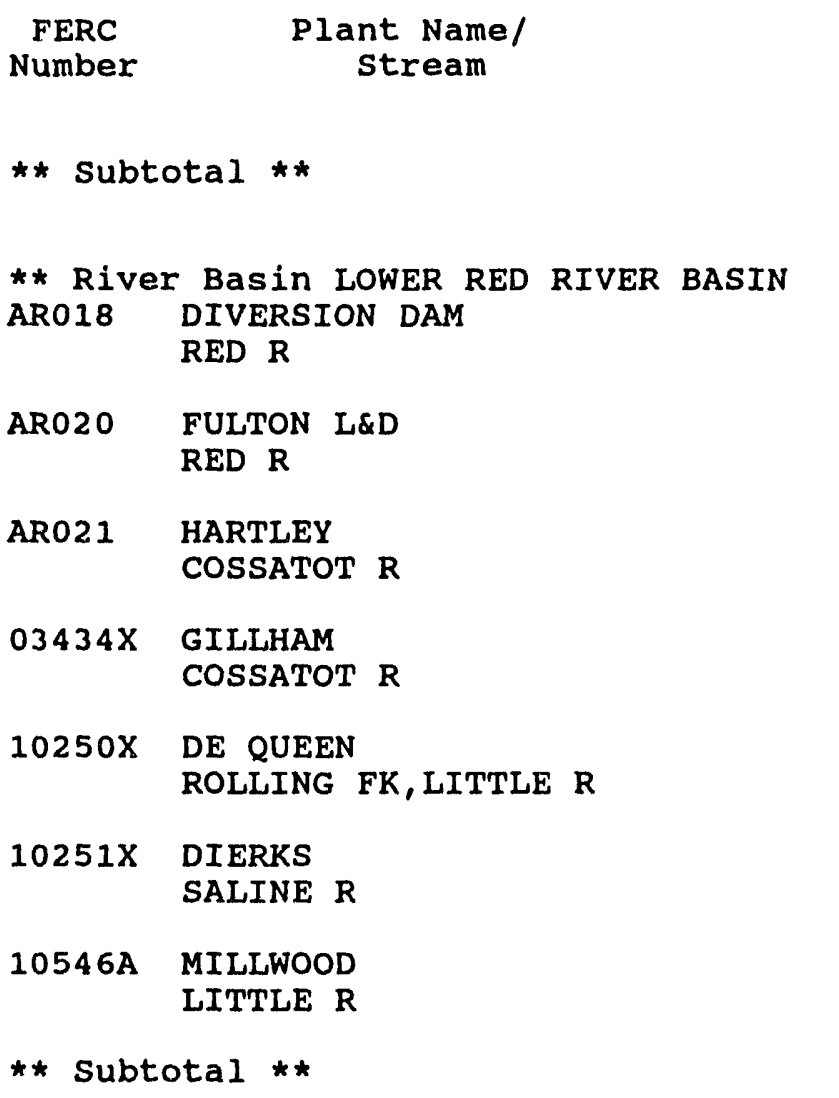

Dam Name Plate

stat Rating (KW) PESF PESF * KW
373250.00

U

U

$\mathbf{U}$

wo

wo

wo

พอ

10

$15000.00 \quad 0.90 \quad 13500.00$

69189.00

43710.10

$\mathrm{U}$

** River Basin LOWER WHITE RIVER BASIN AR012 GUION WHITE $R$

$56900.00 \quad 0.10 \quad 5690.00$

U

$50300.00 \quad 0.50$

25150.00 WHITE $R$

$\mathbf{U}$

$58825.00 \quad 0.50$

$29412 \cdot 50$

WHITE $R$

U

HARDY
SPRING R

$52000.00 \quad 0.10$

5200.00

U

$13830.00 \quad 0.10$

1383.00 WHITE $\mathrm{R}$

U

$125000.00 \quad 0.10$

12500.00

WHITE $R$

U

$19075.00 \quad 0.10$

1907.50 
Page No

$12 / 21 / 93$

FERC

Number

Plant Name/

stream

AR02

BELI FOLEY

STRAWBERRY $R$

AR025 GREERS FERRY

04204A WHITE L\&D 1

WHITE $R$

04659A WHITE L\&D 3

WHITE $R$

04660A WHITE L\&D 2

WHITE $R$

05142X LOWER DAM

LITTLE RED $\mathrm{R}$

05144X UPPER DAM

LITTLE RED $R$

07160X MLMMOTH SPRING

SI'RING R, BLACK $R$

$07964 \times$ DAM 3

SPRING $\mathbf{R}$

$09021 X$ NATIONAL FISH HATCHERY LITTLE RED $\mathbf{R}$

AR019 HILTON DAM 3 SPRING $R$
LITTLE RED $R$

Dam Name plate

stat Rating (KW) PESF

PESF * KW

U

$24000.00 \quad 0.10$

2400.00

W

$0.00 \quad 0.50$

0.00

พั

$6029.00 \quad 0.90$

5426.10

Wo

$10500.00 \quad 0.90$

9450.00

wo

$6307.00 \quad 0.90$

5676.30

พo

$1494.00 \quad 0.90$

1344.60

พั

$1354.00 \quad 0.90$

1218.60

พO

$440.00 \quad 0.50$

$22 \mathrm{c} .00$

wo

$600.00 \quad 0.50$

300.00

wo

$250.00 \quad 0.50$

125.00

พอ

$0.00 \quad 0.50$

0.00

426904.00

107403.60

* * River Basin OUACHITA-BLACK RIVER BASIN $\begin{array}{ll}\text { AROO1 KIRKLAND } & \\ & \text { SMACKOVER CR, OUACHITA } R\end{array}$

U

AR002

CADDO GAP

$\mathbf{U}$

CADDO $\mathrm{R}$

AR003 ROCKPORT

OUACHITA $R$

ARO04 FIDDLERS CREEK

OUACHITA $R$
$\mathbf{U}$

U
$4000.00 \quad 0.90$

$2300.00 \quad 0.50$

$8000.00 \quad 0.90$

7200.00

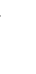




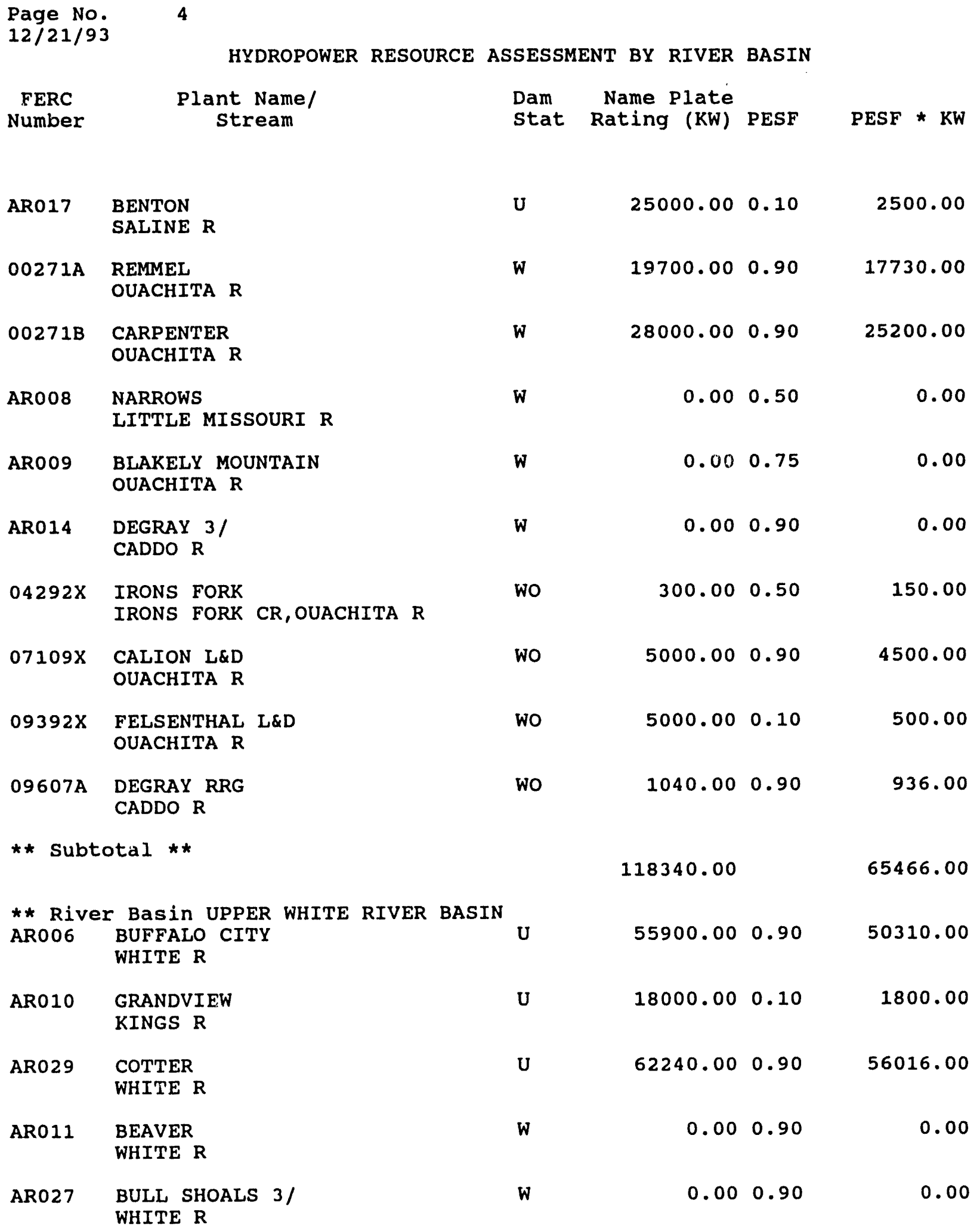


Page No
$12 / 21 / 93$

$\begin{array}{cc}\text { FERC } & \text { Plant Name/ } \\ \text { Number } & \text { Stream }\end{array}$
AR030 NORFORK

NORTH FORK $R$

09020X HATCHERY CONDUIT

NORFORK LAKE, N FK R

W

พo

** Subtotal **

*** Total ***
Dam Name Plate

stat Rating (KW) PESF PESF * KW

$280.00 \quad 0.90$

252.00

221420.00

184878.00

1209103.00
737382.70 
Appendix C

Arkansas (AR) Sites

C-1 
Page No.

$12 / 21 / 93$

FERC

Number

$$
\begin{gathered}
\text { Plant Nane/ } \\
\text { Stream }
\end{gathered}
$$

** State Name AR

** Dam status: W

03043A ARKANSAS L\&D 13

ARKANSAS $\mathbf{R}$

AR005 DARDANELLE

ARKANSAS $R$

AR008 NARROWS

LITTLE MISSOURI $R$

AR009 BLAKELY MOUNTAIN

OUACHITA $\mathbf{R}$

AR011 BEAVER

WHITE $R$

AR014 DEGRAY 3/

AR016 OZARK

ARKANSAS $R$

AR025 GREERS FERRY

LITTLE RED $R$

AR027 BULL SHOALS 3/

WHITE $\mathbf{R}$

AR030 NORFORK

NORTH FORK $R$

03449A MURRAY L\&D 7

ARKANSAS $R$

00271A REMMEL

OUACHITA $R$

00271B CARPENTER

OUACHITA $R$

* Subsubtotal *

** Subtotal **

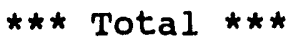

Fed. Name Plate

\begin{tabular}{|c|c|c|c|}
\hline & 0.00 & 0.90 & 0.00 \\
\hline Yes & 35150.00 & 0.90 & 31635.00 \\
\hline Yes & 0.00 & 0.50 & 0.00 \\
\hline Yes & 0.00 & 0.75 & 0.00 \\
\hline Yes & 0.00 & 0.90 & 0.00 \\
\hline Yes & 0.00 & 0.90 & 0.00 \\
\hline Yes & 25600.00 & 0.90 & 23040.00 \\
\hline Yes & 0.00 & 0.50 & 0.00 \\
\hline Yes & 0.00 & 0.90 & 0.00 \\
\hline \multirow[t]{7}{*}{ Yes } & 85000.00 & 0.90 & 76500.00 \\
\hline & 0.00 & 0.90 & 0.00 \\
\hline & 19700.00 & 0.90 & 17730.00 \\
\hline & 28000.00 & 0.90 & 25200.00 \\
\hline & 193450.00 & & 174105.00 \\
\hline & 193450.00 & & 174105.00 \\
\hline & 193450.00 & & 174105.00 \\
\hline
\end{tabular}

Proj Rating (KW) PESF KW * PESF 


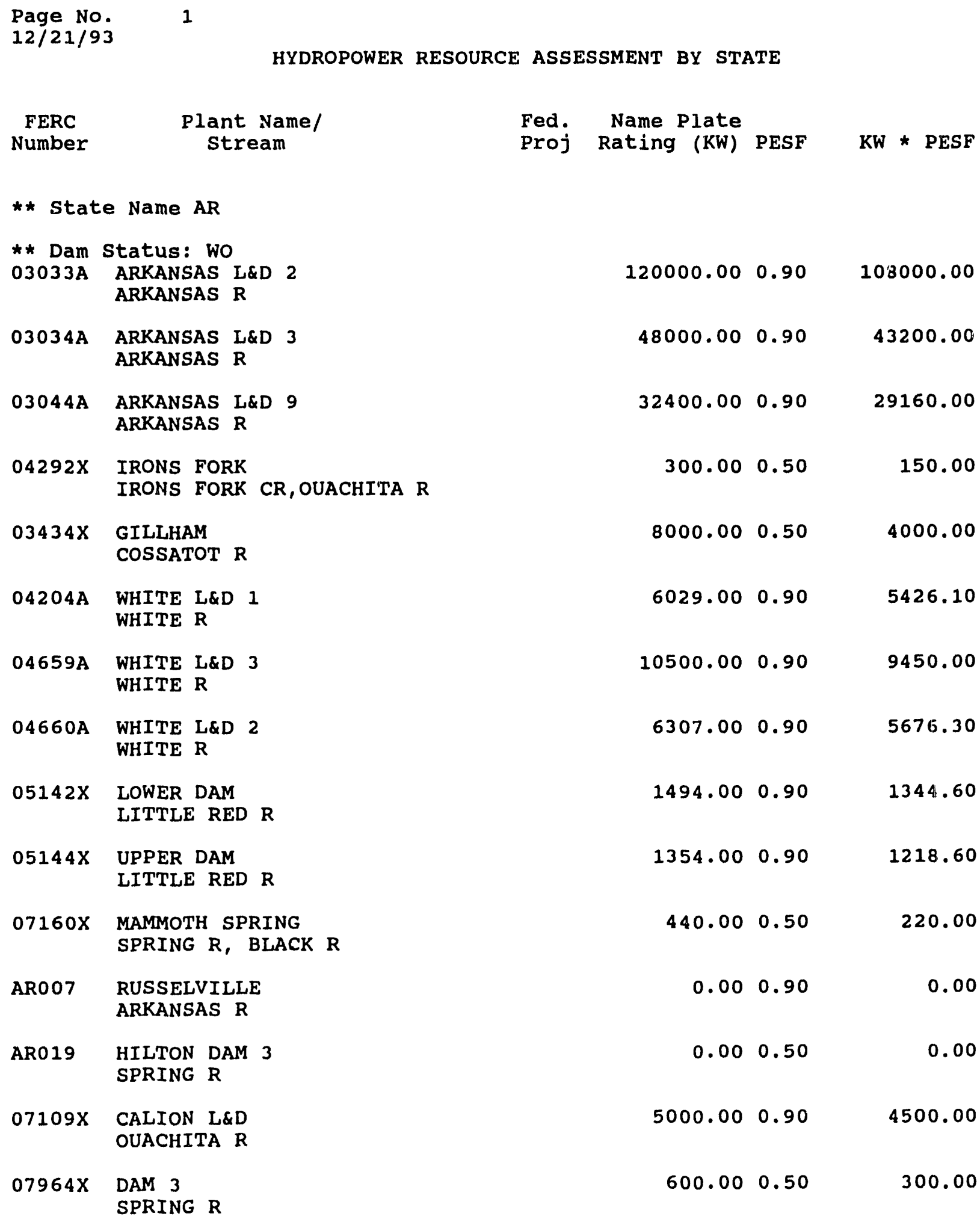

FERC Number

Fed. Name Plate

Proj Rating (KW) PESF KW * PESF 


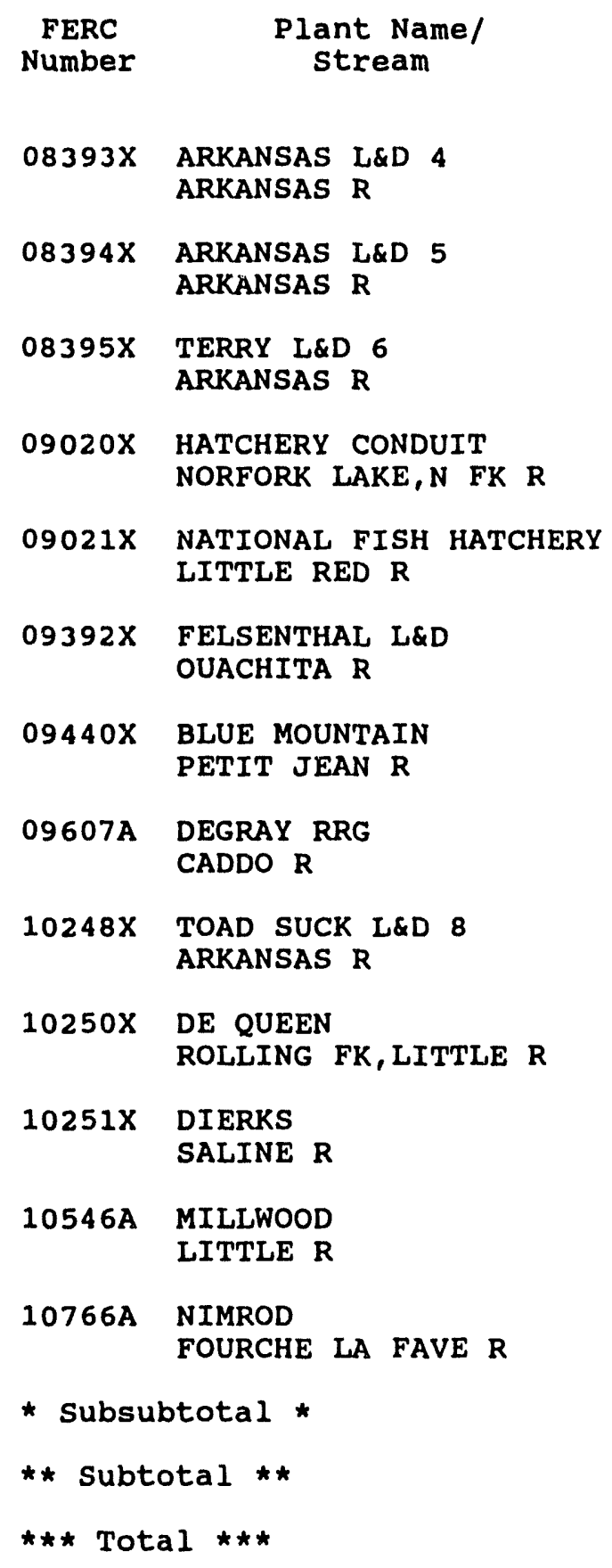

Fed. Name plate

Proj Rating (KW) PESF KW * PESF

\begin{tabular}{|c|c|c|}
\hline 30000.00 & 0.90 & 27000.00 \\
\hline 34000.00 & 0.90 & 30600.00 \\
\hline 34000.00 & 0.90 & 30600.00 \\
\hline 280.00 & 0.90 & 252.00 \\
\hline 250.00 & 0.50 & 125.00 \\
\hline 5000.00 & 0.10 & 500.00 \\
\hline 1600.00 & 0.90 & 1440.00 \\
\hline 1040.00 & 0.90 & 936.00 \\
\hline 10000.00 & 0.90 & 9000.00 \\
\hline 2000.00 & 0.90 & 1800.00 \\
\hline 2000.00 & 0.90 & 1800.00 \\
\hline 15000.00 & 0.90 & 13500.00 \\
\hline 2500.00 & 0.90 & 2250.00 \\
\hline 378094.00 & & 332448.60 \\
\hline 8094.00 & & 332448.60 \\
\hline 378094.00 & & 332448.60 \\
\hline
\end{tabular}




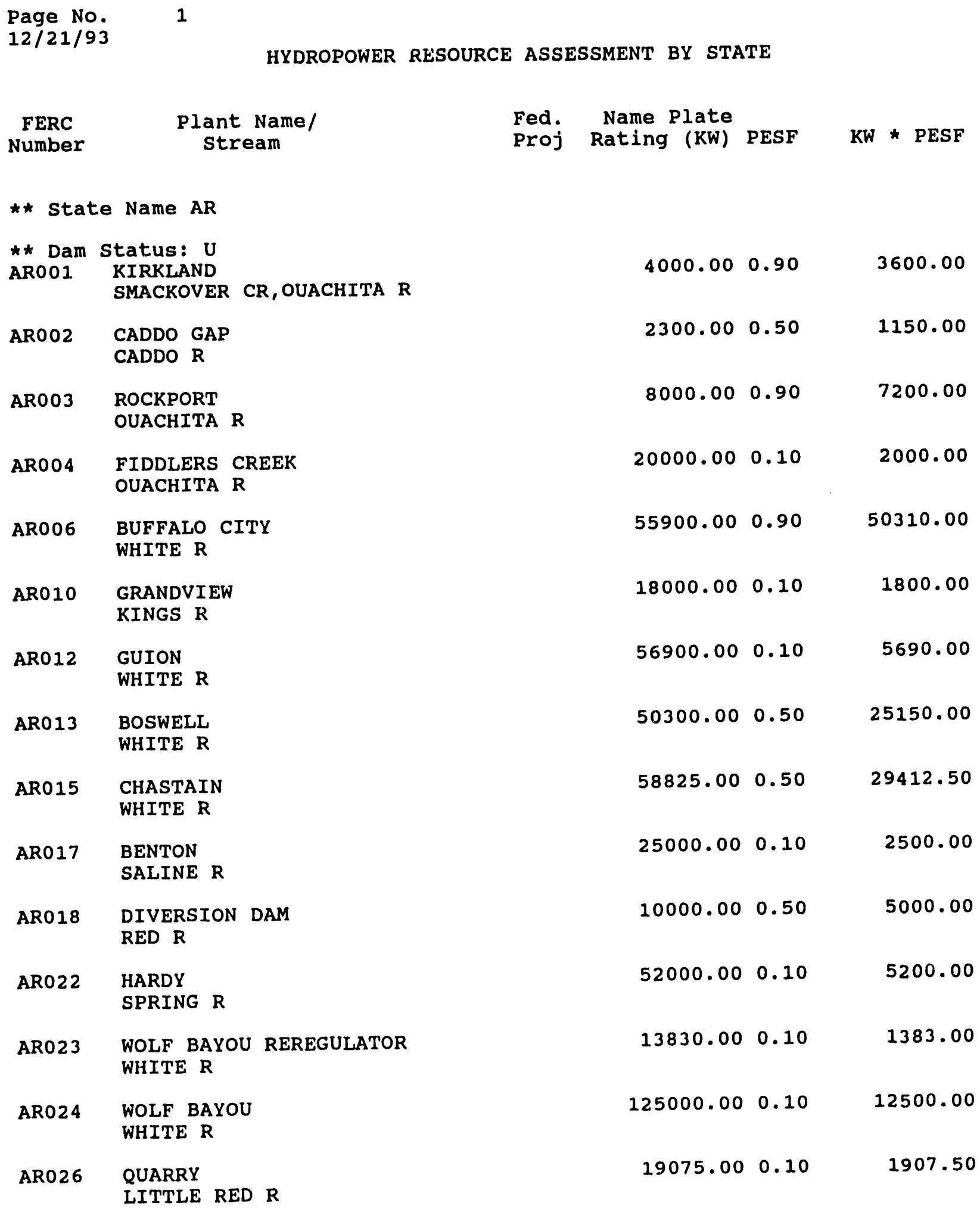




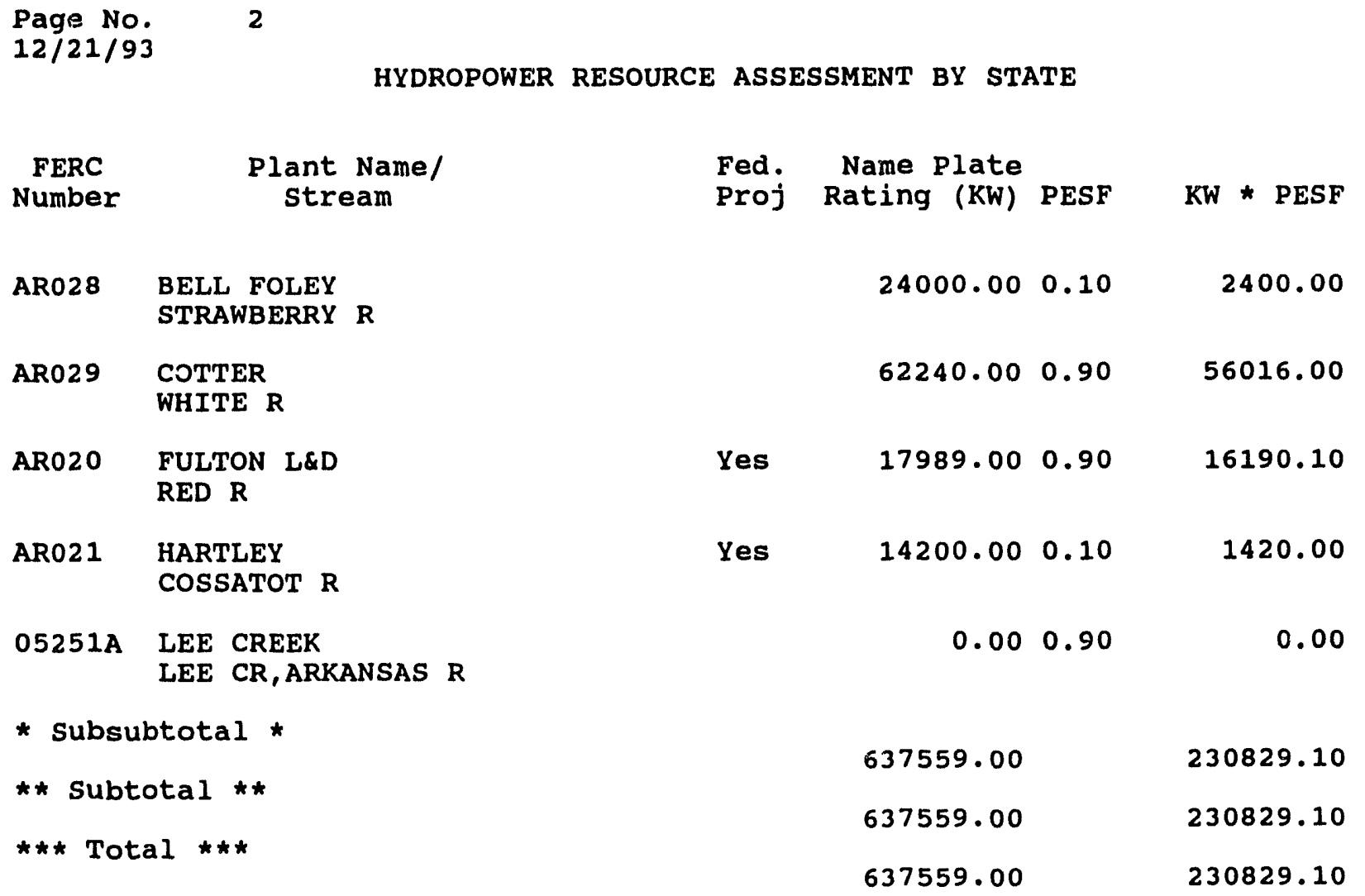




\begin{tabular}{|c|c|c|c|c|}
\hline State & Category & $\begin{array}{l}\text { Number of } \\
\text { Projects }\end{array}$ & $\begin{array}{l}\text { Name Plate } \\
\text { Capacity (KW) }\end{array}$ & $\begin{array}{l}\text { HES Adjusted } \\
\text { Capacity (KW) }\end{array}$ \\
\hline \multirow[t]{4}{*}{$\mathrm{AR}$} & with Power & 13 & 193450 & 174105 \\
\hline & w/O Power & 28 & 378094 & 332448.60 \\
\hline & Undeveloped & 20 & 637559 & 230829.10 \\
\hline & STATE TOTAL & 61 & 1209103 & 737382.70 \\
\hline \multirow[t]{3}{*}{ TOTALS } & With Power & 13 & 193450 & 174105 \\
\hline & w/O Power & 28 & 378094 & 332448.60 \\
\hline & Undeveloped & 20 & 637559 & 230829.10 \\
\hline \multicolumn{2}{|c|}{ GRAND TOTAL } & 61 & 1209103 & 737382.70 \\
\hline
\end{tabular}




\section{Appendix D \\ Resource Database Listing}

D-1 
R E S O U R C E D A T A B A S E L I S T I N G

DATE : $10 / 05 / 93$

PAGE NO: 1

FERC

Number

Plant Name

stream

State

00271 A REMMEL

OUACHITA R

AR

County Name

River Basin

HOT SPRING

OUACHITA-BLACK RIVER BASIN

\begin{tabular}{|c|c|c|c|c|c|c|}
\hline \multirow{3}{*}{\multicolumn{2}{|c|}{$\begin{array}{l}\text { Name Plate } \\
\text { Rating }(\mathrm{KW})\end{array}$}} & \multicolumn{4}{|c|}{ Owner Name } & \multirow[b]{3}{*}{$\begin{array}{l}\text { PESF Annual } \\
\text { Energy Rating }\end{array}$} \\
\hline & & $P$ & ARKANSAS PWF & TR AND LT C & co & \\
\hline & & PESF & PESF $\star K W$ & $\begin{array}{l}\text { Annual } \\
\text { Rating }\end{array}$ & $\begin{array}{l}\text { Energy } \\
\text { (MWh) }\end{array}$ & \\
\hline \multicolumn{2}{|c|}{19700.00} & 0.90 & 17730.00 & \multicolumn{2}{|c|}{3000.00} & 2700 \\
\hline $\begin{array}{l}\text { Unit } \\
\text { Type }\end{array}$ & \multicolumn{2}{|c|}{$\begin{array}{l}\text { Plant } \\
\text { Type }\end{array}$} & $\begin{array}{l}\text { Project } \\
\text { status }\end{array}$ & $\begin{array}{l}\text { Dam } \\
\text { status }\end{array}$ & \multirow[t]{2}{*}{$\begin{array}{l}\text { Latitude } \\
\text { Longitude }\end{array}$} & $\begin{array}{l}3426.00 \\
9254.00\end{array}$ \\
\hline C & \multicolumn{2}{|c|}{ STG } & MO & $\boldsymbol{W}$ & & \\
\hline
\end{tabular}

Factor

Exist Prob

Factor

Exist Prob

Wild/scenic protection wild/Scenic Tributary or Upstream/Downstream

wild/scenic Location

Cultural value

Fish Presence Value

Geologic Value

Historic Value

other Value

Recreation Value

Scenic Value

\begin{tabular}{c} 
Exist Prob \\
\hline 0.90 \\
0.90 \\
0.90 \\
0.90 \\
0.90 \\
0.90 \\
0.90 \\
0.90
\end{tabular}

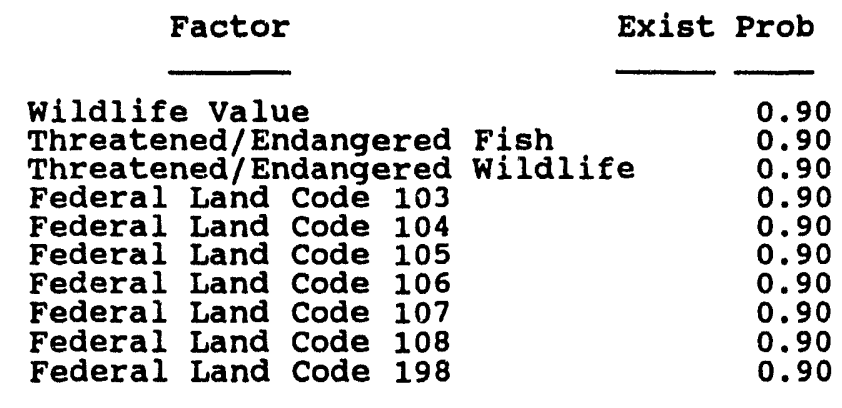


R E S O U R C E

DATE: $10 / 05 / 93$

FERC

Number

$00271 B$

CARPENTER

County Name

GARLAND
D A T A B A S E

L I S T I N G

PAGE NO: 2

stream

State

Name

OUACHITA $R$

AR

River Basin

OUACHITA-BLACK RIVER BASIN

Class Owner Name

P ARKANSAS PWR AND LT CO

Name Plate

PES

Rating (KW)

PESF

PESF*KW

Annual Energy

28000.00

0.90

25200.00

61340.00

PESF Annual

Energy Rating (MWh)

55206

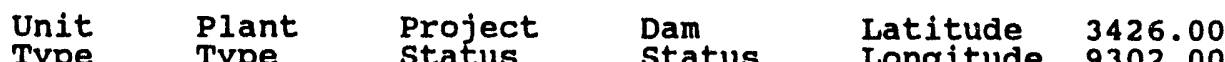

Typ

STG

MO

พ

Factor

Exist Prob

Factor

Exist Prob

Wild/Scenic Protection

Wild/Scenic Tributary or

Upstream/Downstream wild/Scenic Location

0.90
0.90
0.90
0.90
0.90
0.90
0.90
0.90
0.90

Wildlife Value

Threatened/Endangered Fish

Threatened/Endangered Wildlife

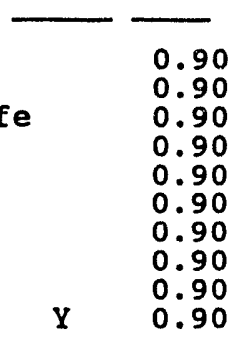

Cultural value

Fish Presence Value

Geologic Value

Federal Land Code 103

Federal Land Code 104

Federal Land Code 105

Federal Land Code 106

Federal Land Code 107

Federal Land Code 108

Recreation Value

Federal Land Code 198

0.90

0.90

0.90

0.90

0.90 

R E S O U R C E
D A T A B A S E
I I S T I N G

DATE: $10 / 05 / 93$

PAGE NO: 3

FERC

Number

Plant Name

03033A ARKANSAS LED 2

County Name

DESHA

\begin{tabular}{|c|c|c|c|c|c|c|c|}
\hline \multirow{3}{*}{\multicolumn{2}{|c|}{$\begin{array}{l}\text { Name Plate } \\
\text { Rating (KW) }\end{array}$}} & \multicolumn{5}{|c|}{ Owner Name } & \multirow[b]{3}{*}{$\begin{array}{c}\text { PESF Ar } \\
\text { Energy Rat }\end{array}$} \\
\hline & & C & ARKANSAS & ELEC & COOP CO & $\mathbf{R P}$ & \\
\hline & & PESF & \multicolumn{2}{|c|}{$P E S F \star K W$} & $\begin{array}{l}\text { Annual } \\
\text { Rating }\end{array}$ & $\begin{array}{l}\text { Energy } \\
\text { (MWh) }\end{array}$ & \\
\hline \multicolumn{2}{|c|}{120000.00} & 0.90 & 108000.00 & & \multicolumn{2}{|c|}{340000.00} & 306000 \\
\hline $\begin{array}{l}\text { Unit } \\
\text { Type }\end{array}$ & \multicolumn{2}{|c|}{$\begin{array}{l}\text { Plant } \\
\text { Type }\end{array}$} & $\begin{array}{l}\text { Project } \\
\text { status }\end{array}$ & $\begin{array}{l}\text { Dam } \\
\text { sta }\end{array}$ & atus & \multirow[t]{2}{*}{$\begin{array}{l}\text { Latitude } \\
\text { Longitude }\end{array}$} & \multirow[t]{2}{*}{$\begin{array}{l}3359.00 \\
9112.00\end{array}$} \\
\hline C & \multicolumn{2}{|c|}{ ROR } & MO & wo & & & \\
\hline
\end{tabular}

Factor

Exist Prob

0.90

Wild/Scenic Protection

wild/Scenic Tributary or

Upstream/Downstream

wild/Scenic Location

Cultural value

Fish Presence Value

Geologic value

Historic Value

other value

Recreation value

Scenic Value

$\begin{array}{cc}\text { stream } & \begin{array}{c}\text { State } \\ \text { Name }\end{array} \\ \text { ARKANSAS } R & \text { AR } \\ \text { River Basin } & \end{array}$

LOWER ARKANSAS RIVER BASIN 
RES O U R C E D A T A B A S E I I S T I N G

DATE: $10 / 05 / 93$

PAGE NO: 4

FERC

Number

plant Name

stream

state

03034A ARKANSAS L\&D 3

ARKANSAS R

AR

County Name

River Basin

LINCOLN

LOWER ARKANSAS RIVER BASIN

Class Owner Name

C ARKANSAS ELEC COOP CORP

\begin{tabular}{|c|c|c|c|c|c|c|}
\hline \multicolumn{2}{|c|}{$\begin{array}{l}\text { Name Plate } \\
\text { Rating (KW) }\end{array}$} & PESF & $P E S F \star K T$ & $\begin{array}{l}\text { Ann } \\
\text { Rat }\end{array}$ & $\begin{array}{l}\text { Energy } \\
\text { (MWh) }\end{array}$ & $\begin{array}{l}\text { PESF Annual } \\
\text { Energy Rating (MWh) }\end{array}$ \\
\hline \multicolumn{2}{|c|}{48000.00} & 0.90 & 43200.00 & \multicolumn{2}{|c|}{155000.00} & 139500 \\
\hline $\begin{array}{l}\text { Unit } \\
\text { Type }\end{array}$ & \multicolumn{2}{|c|}{$\begin{array}{l}\text { Plant } \\
\text { Type }\end{array}$} & $\begin{array}{l}\text { Project } \\
\text { status }\end{array}$ & $\begin{array}{l}\text { Dam } \\
\text { status }\end{array}$ & \multirow[t]{2}{*}{$\begin{array}{l}\text { Latitude } \\
\text { Longitude }\end{array}$} & $\begin{array}{l}3410.00 \\
9141.00\end{array}$ \\
\hline C & \multicolumn{2}{|c|}{ ROR } & MO & \multicolumn{2}{|l|}{ พ० } & \\
\hline
\end{tabular}

Factor

Exist Prob

Factor

Exist Prob

Wild/Scenic Protection

wild/Scenic Tributary or

Upstream/Downstream

wild/Scenic Location

Cultural value

Fish Presence Value

Geologic Value

Historic Value

other Value

Recreation value

scenic Value

0.90
0.90
0.90
0.90
0.90
0.90
0.90
0.90
0.90

Wildlife Value

Threatened/Endangered Fish

Threatened/Endangered Wildlife

Federal Iand Code 103

Federal Land Code 104

Federal Land Code 105

Federal Land Code 106

Federal Land Code 107

Federal Land Code 108

0.90

0.90

0.90

0.90

0.90

0.90

0.90

0.90

Federal Land Code 198

0.90

0.90

$\mathbf{Y}$

0.90 

R E S O U R C E
D A T A B A E
L I S T I N G

DATE: $10 / 05 / 93$

PAGE NO: 5

FERC

Number

Plant Name

stream

state

03043A ARKANSAS L\&D 13

County Name

ARKANSAS $R$

AR

River Basin

CRAWFORD

LOWER ARKANSAS RIVER BASIN

\begin{tabular}{|c|c|c|c|c|c|}
\hline \multirow[b]{3}{*}{$\begin{array}{l}\text { Name plate } \\
\text { Rating (KW) }\end{array}$} & \multicolumn{4}{|c|}{ Owner Name } & \\
\hline & C & ARKANSAS ELEC & COOP & CORP, ET AL & \\
\hline & PESF & $P E S F \star K W$ & $\begin{array}{l}\text { Annua } \\
\text { Ratin }\end{array}$ & $\begin{array}{l}\text { il Energy } \\
\text { ig (MWh) }\end{array}$ & $\begin{array}{l}\text { PESF Annual } \\
\text { Energy Rating }\end{array}$ \\
\hline 0.00 & 0.90 & 0.00 & & 0.00 & 0 \\
\hline
\end{tabular}

Unit Plant Project Dam Latitude 3521.00

Type Type Status status Longitude $\mathbf{9 4 1 8 . 0 0}$

ROR

MO

$\boldsymbol{W}$

Factor

Exist Prob

Factor

Exist Prob

Wild/Scenic Protection

wild/Scenic Tributary or Upstream/Downstream wild/Scenic Location Cultural Value

Fish Presence Value

Geologic Value

Historic Value

other value

Recreation value

Scenic Value

0.90

0.90
0.90
0.90
0.90
0.90
0.90
0.90
0.90

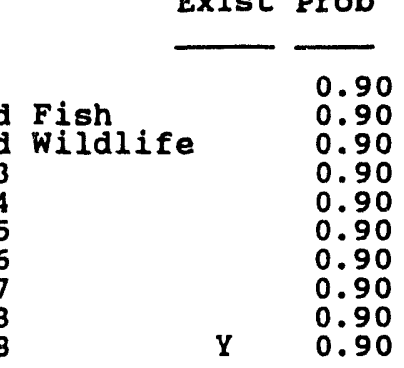


RE S O UR CE DA TABASE L I S T I N G

DATE: $10 / 05 / 93$

PAGE NO: 6

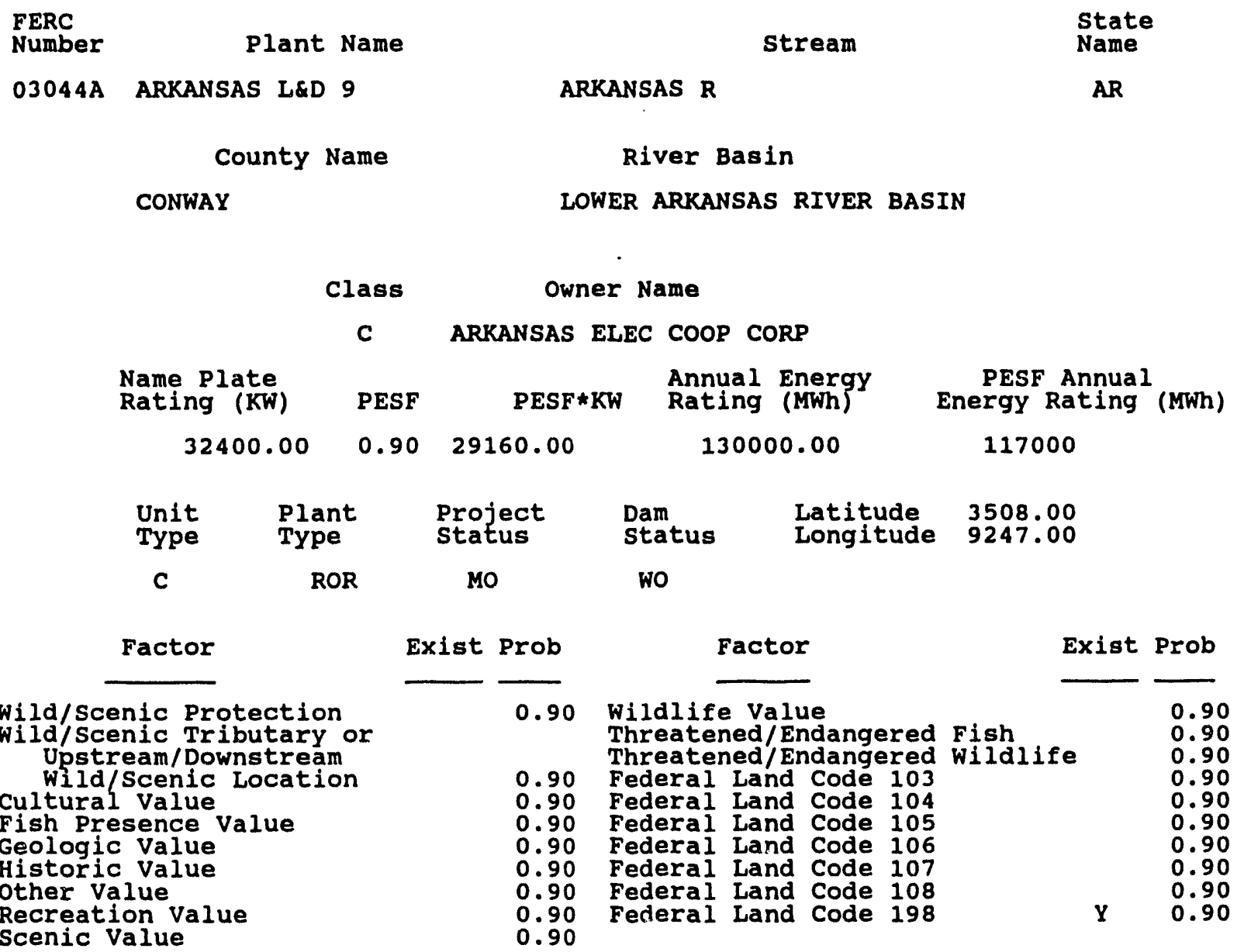



R E S O U R C E
D A T A B A S E
L I $S$ T I N G

DATE: $10 / 05 / 93$

FERC

Number

Plant Name

PAGE NO: 7

$03434 X$ GILLHAM

COSSATOT $\mathbf{R}$

state

stream

Name

AR

County Name

River Basin

HOWARD

LOWER RED RIVER BASIN

\begin{tabular}{|c|c|c|c|c|c|c|}
\hline \multirow{3}{*}{\multicolumn{2}{|c|}{$\begin{array}{l}\text { Name Plate } \\
\text { Rating (KW) }\end{array}$}} & \multicolumn{4}{|c|}{ Owner Name } & \multirow[b]{3}{*}{$\begin{array}{l}\text { PESF Annual } \\
\text { Energy Rating (MWh) }\end{array}$} \\
\hline & & $\mathbf{M}$ & HOPE, CITY & OF & & \\
\hline & & PESF & PESF*KW & $\begin{array}{l}\text { Annual } \\
\text { Rating }\end{array}$ & $\begin{array}{l}\text { Energy } \\
\text { (MWh) }\end{array}$ & \\
\hline \multicolumn{2}{|c|}{8000.00} & 0.50 & 4000.00 & \multicolumn{2}{|c|}{15567.00} & 7783.50 \\
\hline $\begin{array}{l}\text { Unit } \\
\text { Type }\end{array}$ & $\begin{array}{l}\text { Pli } \\
\text { TYl }\end{array}$ & & $\begin{array}{l}\text { Project } \\
\text { status }\end{array}$ & $\begin{array}{l}\text { Dam } \\
\text { status }\end{array}$ & $\begin{array}{l}\text { Latitude } \\
\text { Longitude }\end{array}$ & $\begin{array}{l}3432.00 \\
9414.00\end{array}$ \\
\hline C & & & $x x$ & พอ & & \\
\hline
\end{tabular}

Factor

Exist Prob

Factor

Exist Prob

Wild/Scenic Protection

Wild/Scenic Tributary or Upstream/Downstream

wild/Scenic Location

\begin{tabular}{cc} 
& \\
\cline { 2 - 2 } & 0.90 \\
& \\
& $C \quad 10$ \\
& 0.90 \\
$Y$ & 0.75 \\
$Y$ & 0.90 \\
& 0.90 \\
$Y$ & 0.90 \\
$Y$ & 0.75 \\
$Y$ & 0.90
\end{tabular}

Factor
Wildlife Value
Threatened/Endangered
Threatened/ Endangered
Federal Land Code 103
Federal Land Code 104
Federal Land Code 105
Federal Land Code 106
Federal Land Code 107
Federal Land Code 108
Federal Land Code 198

\begin{tabular}{|c|c|c|}
\hline $\begin{array}{l}\text { Fish } \\
\text { wild ife }\end{array}$ & $\mathbf{Y}$ & $\begin{array}{l}0.75 \\
0.90 \\
0.90 \\
0.90 \\
0.90 \\
0.90 \\
0.90 \\
0.90 \\
0.90 \\
0.90\end{array}$ \\
\hline
\end{tabular}

Fish Presence Value

Geologic Value

Historic Value

other Value

Recreation Value

Scenic Value

0.90

Federal Land Code 198 
RE S O UR C E D A T A B A S E L I S T I N G

DATE: $10 / 05 / 93$

PAGE NO: 8

FERC

Number

03449A MURRAY L\&D 7

County Name

PULASKI
Plant Name

Class

$\mathbf{M}$

Owner Name

ARKANSAS $\mathbf{R}$

stream

state

Name

AR

$$
\text { River Basin }
$$

LOWER ARKANSAS RIVER BASIN

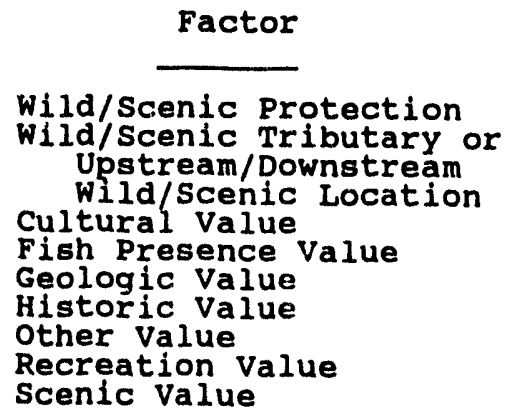

Exist Prob

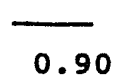

0.90

0.90

0.90

0.90

0.90

0.90

0.90

0.90

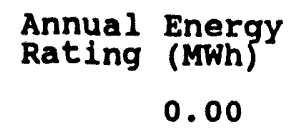


R E S O U R C E

DATE: $10 / 05 / 93$

FERC

Number

04204A

WHITE L\&D 1

County Name

INDEPENDENCE
D A T A B A $E$

L I S T I N G

PAGE NO: 9

stream

WHITE $R$

River Basin

LOWER WHITE RIVER BASIN

\section{Class Owner Name}

M BAtESVILLE, CITY OF

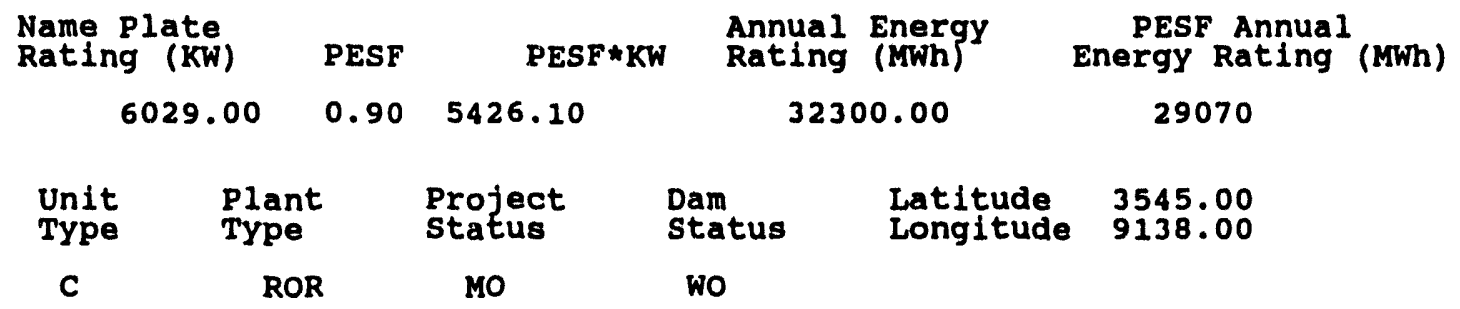

Factor

Exist Prob

Factor

Exist Prob

Wild/Scenic Protection

Wild/Scenic Tributary or Upstream/Downstream wild/Scenic Location

0.90


0.90
0.90
0.90
0.90
0.90
0.90
0.90
0.90

\begin{tabular}{|c|c|c|c|}
\hline & Factor & Exist & Prob \\
\hline $\begin{array}{l}\text { Wildlife } \\
\text { Threaten } \\
\text { Threaten } \\
\text { Federal } \\
\text { Federal } \\
\text { Federal } \\
\text { Federal } \\
\text { Federal } \\
\text { Federal } \\
\text { Federal }\end{array}$ & $\begin{array}{l}\text { e Value } \\
\text { ned/Endangered } \\
\text { ned/Endangered } \\
\text { Land Code } 103 \\
\text { Land Code } 104 \\
\text { Land Code } 105 \\
\text { Land Code } 106 \\
\text { Land Code } 107 \\
\text { Land Code } 108 \\
\text { Land Code } 198\end{array}$ & $\begin{array}{l}\text { Fish } \\
\text { Wildilfe }\end{array}$ & $\begin{array}{l}0.90 \\
0.90 \\
0.90 \\
0.90 \\
0.90 \\
0.90 \\
0.90 \\
0.90 \\
0.90 \\
0.90\end{array}$ \\
\hline
\end{tabular}

Fish Presence Value

Geologic Value

Historic Value

other value

Recreation Value

Scenic Value

0.90 
R E S O U R C E D A T A B A S E L I S T I N G

DATE: $10 / 05 / 93$

PAGE NO: 10

FERC

Number

Plant Name

04292X IRONS FORK

IRONS FORK CR, OUACHITA $R$

stream

Name

County Name

River Basin

POLK

OUACHITA-BLACK RIVER BASIN

\begin{tabular}{|c|c|c|c|c|c|}
\hline \multirow[b]{3}{*}{$\begin{array}{l}\text { Name plate } \\
\text { Rating (KW) }\end{array}$} & \multicolumn{4}{|c|}{ Owner Name } & \multirow[b]{3}{*}{$\begin{array}{l}\text { PESF Annual } \\
\text { Energy Rating (MWh) }\end{array}$} \\
\hline & C & ARKANSAS EL & coop $\mathrm{C}$ & ORP & \\
\hline & PESF & $P E S F \star K W$ & $\begin{array}{l}\text { Annual } \\
\text { Rating }\end{array}$ & $\begin{array}{l}\text { Energy } \\
\text { (MWh) }\end{array}$ & \\
\hline 300.00 & 0.50 & 150.00 & & 75.00 & 137.50 \\
\hline $\begin{array}{l}\text { Unit } \\
\text { Type }\end{array}$ & & $\begin{array}{l}\text { Project } \\
\text { status }\end{array}$ & $\begin{array}{l}\text { m } \\
\text { atus }\end{array}$ & $\begin{array}{l}\text { Latitude } \\
\text { Longitude }\end{array}$ & $\begin{array}{l}3429.00 \\
9415.00\end{array}$ \\
\hline c & & $x x$ & & & \\
\hline
\end{tabular}

Factor

Exist Prob

Factor

Exist Prob

wild/scenic Protection

wild/Scenic Tributary or

Upstream/Downstream

wild/Scenic Location

Cultural value

Fish Presence Value

Geologic Value

Historic Value

other Value

Recreation Value

Scenic Value

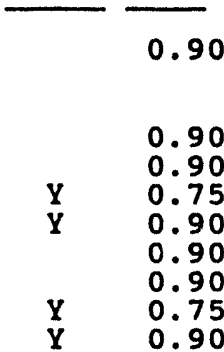

Wildlife Value
Threatened/Endangered Fish
Threatened/Endangered Wildlife
Federal Land Code 103
Federal Land Code 104
Federal Land Code 105
Federal Land Code 106
Federal Land Code 107
Federal Land Code 108
Federal Land Code 198

0.75

0.90

0.90

0.90

0.75

0.90

0.90

0.90

0.90

0.90 
RES O URCE DATA BASE L I S T I N G

DATE: $10 / 05 / 93$

PAGE NO: 11

FERC

Numliar

Plant Name

stream

State

04659A WHITE LED 3

WHITE $R$

Name

County Name

River Basin

INDEP ENDENCE

LOWER WHITE RIVER BASIN

Class Owner Name

M INDEPENDENCE, COUNTY OF

$\begin{array}{ccccc}\begin{array}{l}\text { Name Plate } \\ \text { Rating }\end{array} \text { (KW) } & \text { PESF } & \text { PESF*KW } & \begin{array}{c}\text { Annual Energy } \\ \text { Rating }\end{array} \text { (MWh) } & \begin{array}{c}\text { PESF Annual } \\ \text { Energy Rating (MWh) }\end{array} \\ 10500.00 & 0.90 & 9450.00 & 37668.00 & 33901.20\end{array}$

\begin{tabular}{|c|c|c|c|}
\hline $\begin{array}{l}\text { Unit } \\
\text { Type }\end{array}$ & Plant & $\begin{array}{l}\text { Project } \\
\text { status }\end{array}$ & $\begin{array}{l}\text { Dam } \\
\text { status }\end{array}$ \\
\hline
\end{tabular}

C

ROR

MO

wo

Factor

Wild/Scenic Protection

Wild/Scenic Tributary or Upstream/Downstream wild/Scenic Location Cultural value

Fish Presence Value

Geologic Value

ulistoric Value

other Value

Recreation Value

Scenic Value
Exist Prob

0.90

0.90

0.90

0.90

0.90

0.90

0.90

0.90

0.90

\begin{tabular}{lll}
\multicolumn{1}{c}{ Factor } & \multicolumn{1}{c}{ Exist } & Prob \\
Wildilfe Value & & 0.90 \\
Threatened/Endangered Fish & 0.90 \\
Threatened/Endangered Wildlife & 0.90 \\
Federal Land Code 103 & 0.90 \\
Federal Land Code 104 & 0.90 \\
Federal Land Code 105 & 0.90 \\
Federal Land Code 106 & 0.90 \\
Federal Land Code 107 & 0.90 \\
Federal Land Code 108 & & 0.90 \\
Federal Land Code 198 & $Y$ & 0.90
\end{tabular}


RESOUR CE DATABAS E L I S T I N G

DATE: $10 / 05 / 93$

PAGE NO: 12

FERC

Number

Plant Name

04660A WHITE LED 2

County Name

INDEPENDENCE stream

WHITE $R$

River Basin

LOWER WHITE RIVER BASIN

\begin{tabular}{|c|c|c|c|c|}
\hline & Class & \multicolumn{2}{|c|}{ Owner Name } & \\
\hline & $\mathbf{M}$ & INDEPENDENCE, & COUNTY OF & \\
\hline $\begin{array}{l}\text { Name Plate } \\
\text { Rating (KW) }\end{array}$ & PESF & PESF $\star K W$ & $\begin{array}{l}\text { Annual Energy } \\
\text { Rating (MWh) }\end{array}$ & $\begin{array}{l}\text { PESF Annual } \\
\text { Energy Rating (MWh) }\end{array}$ \\
\hline 6307.00 & 0.90 & 5676.30 & 38300.00 & 34470 \\
\hline
\end{tabular}

$\begin{array}{cccccc}\begin{array}{c}\text { Unit } \\ \text { Type }\end{array} & \begin{array}{c}\text { Plant } \\ \text { Type }\end{array} & \begin{array}{c}\text { Project } \\ \text { Status }\end{array} & \begin{array}{l}\text { Dam } \\ \text { Status }\end{array} & \begin{array}{l}\text { Latitude } \\ \text { Longitude }\end{array} & 3537.00 \\ \mathbf{9 1 4 5 . 0 0 0} & \\ \text { C } & \text { ROR } & \text { MO } & \text { wo } & \end{array}$

\author{
Factor \\ Wild/Scenic Protection \\ wild/Scenic Tributary or \\ Upstream/Downstream \\ wild/Scenic Location \\ Cultural Value \\ Fish Presence Value \\ Geologic Value \\ Historic Value \\ other Value \\ Recreation value \\ Scenic Value
}

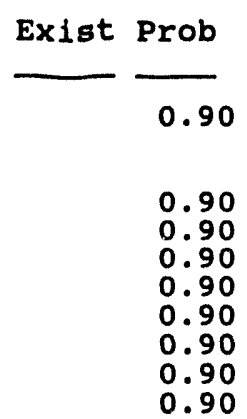

\begin{tabular}{|c|c|c|c|}
\hline & Factor & Exist & Prob \\
\hline $\begin{array}{l}\text { Wildlife } \\
\text { Threaten } \\
\text { Threaten } \\
\text { Federal } \\
\text { Federal } \\
\text { Federal } \\
\text { Federal } \\
\text { Federal } \\
\text { Federal } \\
\text { Federal }\end{array}$ & $\begin{array}{l}\text { Value } \\
\text { ned/Endangered } \\
\text { ned/Endangered } \\
\text { Land Code } 103 \\
\text { Land Code } 104 \\
\text { Land Code } 105 \\
\text { Land Code } 106 \\
\text { Land Code } 107 \\
\text { Land Code } 108 \\
\text { Land Code } 198\end{array}$ & $\begin{array}{l}\text { Fish } \\
\text { Wildifife }\end{array}$ & $\begin{array}{l}0.90 \\
0.90 \\
0.90 \\
0.90 \\
0.90 \\
0.90 \\
0.90 \\
0.90 \\
0.90 \\
0.90\end{array}$ \\
\hline
\end{tabular}



R E S O U R C E
D A T A B A S E
L I S T I N G

DATE : $10 / 05 / 93$

FERC

Number

Plant Name

05142X LOWER DAM

County Name WHITE

PAGE NO: 13

LITTLE RED $R$ stream $\quad \begin{gathered}\text { State } \\ \text { Name }\end{gathered}$

River Basin

LOWER WHITE RIVER BASIN

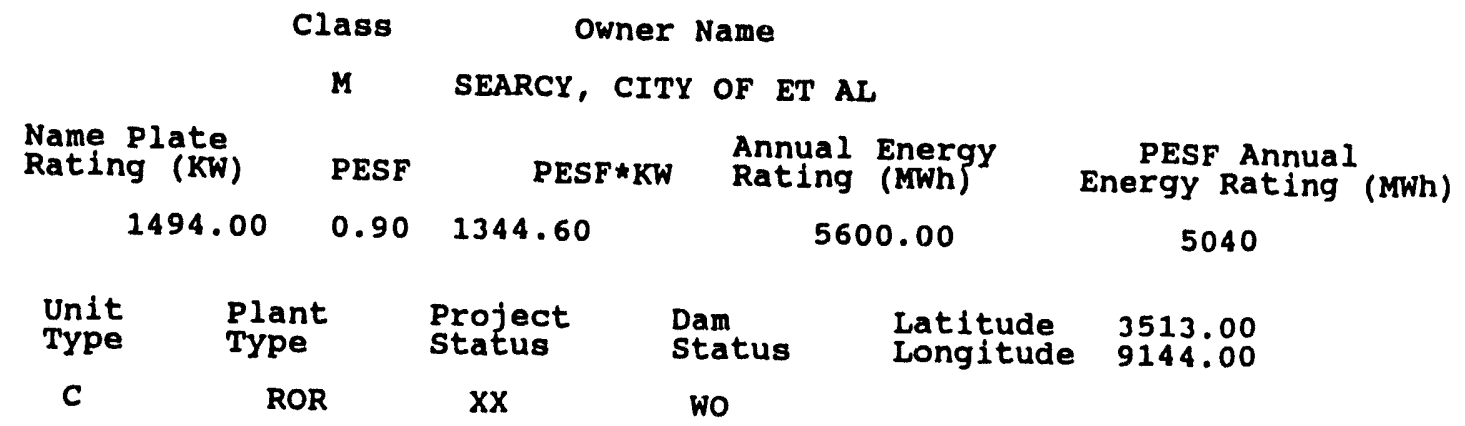

Factor

Exist Prob

Factor

Exist Prob

wild/Scenic Protection

wild/Scenic Tributary or Upstream/Downstream wild/Scenic Location

0.90
0.90
0.90
0.90
0.90
0.90
0.90
0.90
0.90

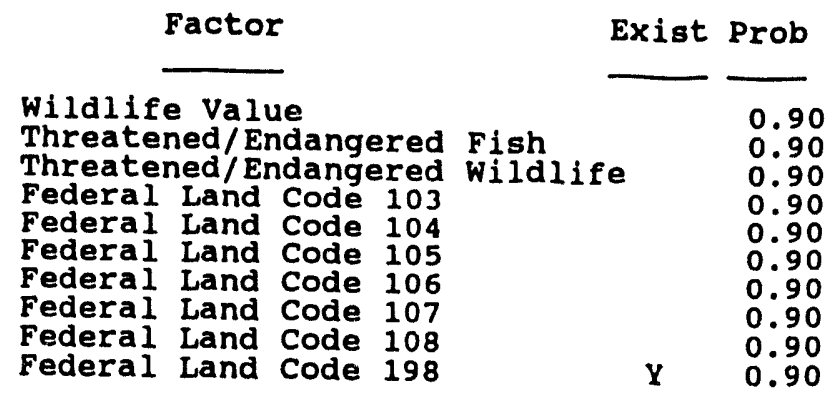

Fish Presence value

Geologic value

Historic Value

other Value

Recreation Value

0.90

Wildlife Value

Threatened/Endangered Fish

0.90

Federal Land Code 103

Federal Land Code 104

Federal Land Code 105

Federal Land Code 108

Federal Land Code 198

0.90 
RE S O U R C E D A T A B A S E L I S T I N G

DATE: $10 / 05 / 93$

\author{
FERC \\ Number \\ Plant Name \\ $05144 X$ UPPER DAM
}

County Name

WHITE
PAGE NO: 14

$\begin{array}{lc}\text { Stream } & \begin{array}{c}\text { State } \\ \text { Name }\end{array} \\ \text { LITTLE RED R } & \text { AR } \\ \text { River Basin } & \\ \text { LOWER WHITE RIVER BASIN } & \end{array}$

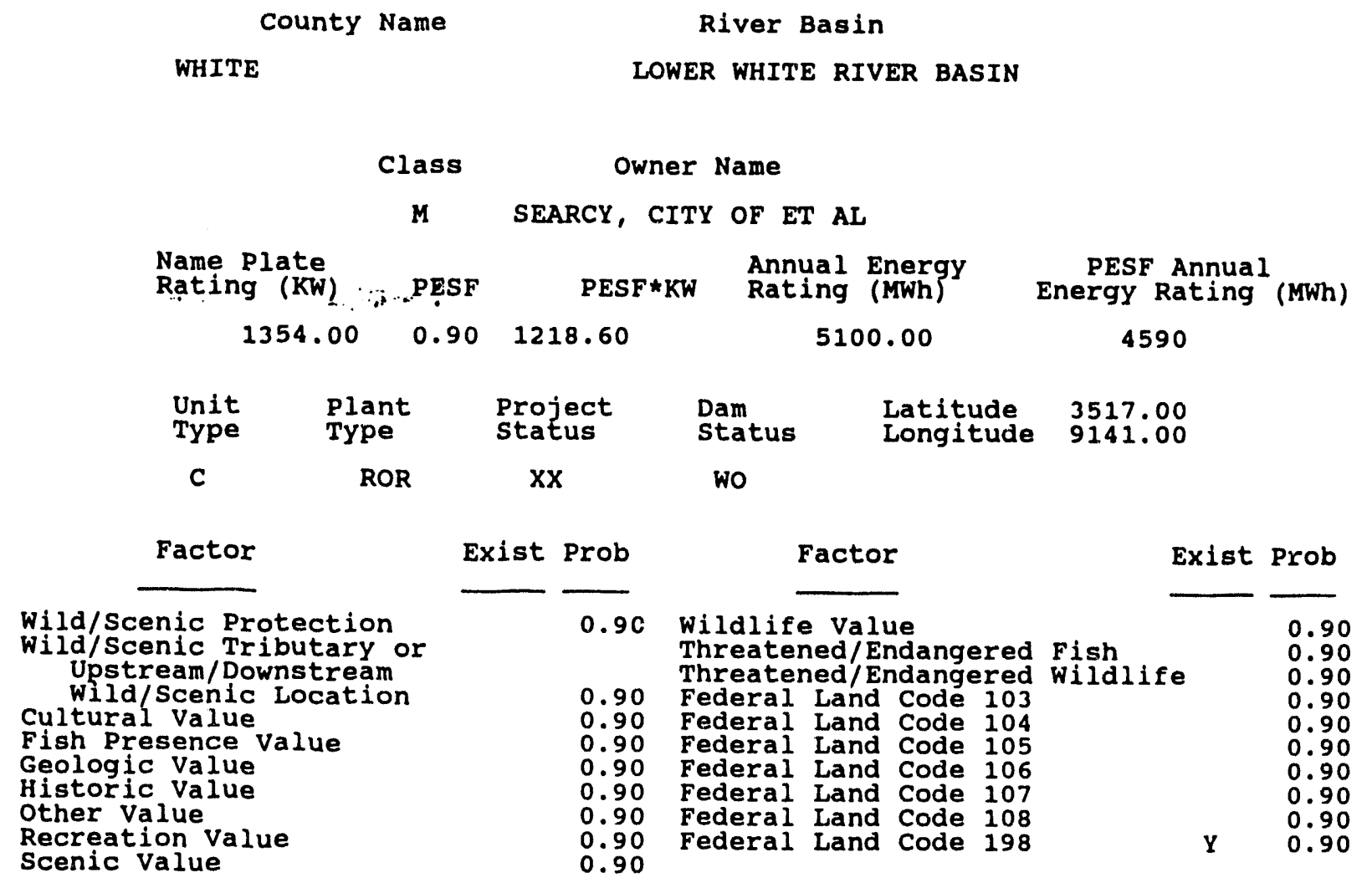


R E S O U C E D A T A B S E L I S T I N G

DATE: $12 / 05 / 93$

FERC

Number

$05251 \mathrm{~A}$
Plant Name

LEE CREEK

County Name

CRAWFORD

PAGE NO: 15

stream

LEE CR, ARKANSAS $R$

River Basin

LOWER ARKANSAS RIVER BASIN

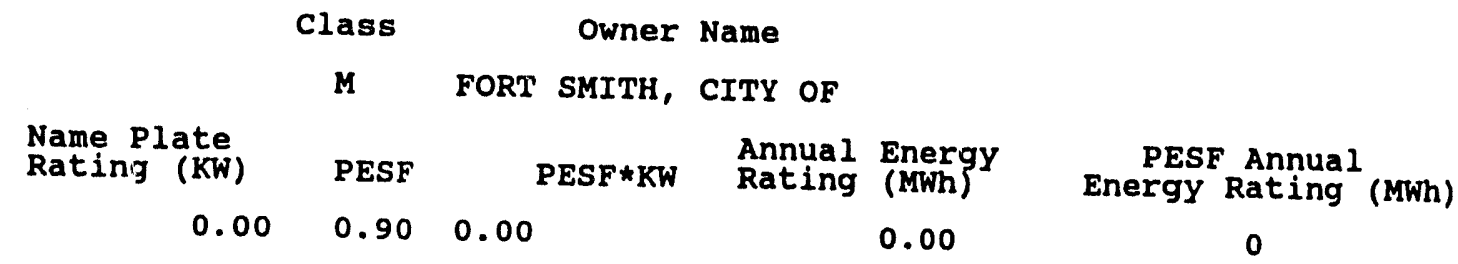

$\begin{array}{cccccc}\text { Unit } & \text { Plant } & \text { Project } & \text { Dam } & \text { Latitude } & 3529.00 \\ \text { Type } & \text { Type } & \text { Status } & \text { Status } & \text { Longitude } & \mathbf{9 4 1 7 . 0 0 0} \\ \text { Z } & \text { ROR } & \text { NO } & \text { U } & & \end{array}$

Factor

Exist Prob

Factor

Exist Prob

Wild/Scenic Protection

Wild/Scenic Tributary or

Upstream/Downstream

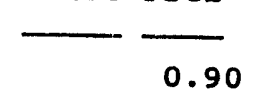

Wild/Scenic Location

Threatened/Endangered $\mathrm{Fish}$

0.90 Federal Land Code 103 Wildlife

State

Name

AR

Cultural value

Fish Presence Value

Geologic Value

Historic Value

other Value

Recreation Value

Scenic Value

0.90

Federal Land Code 104

0.90 Federal Land Code 105

0.90 Federal Land Code 107

0.90 Federal Land Code 108

0.90 Federal Land Code 198

0.90

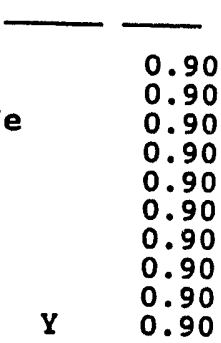



RESOUR C E
DA T A B S E
L I S T I G

DATE: $10 / 05 / 93$

PAGE NO: 16

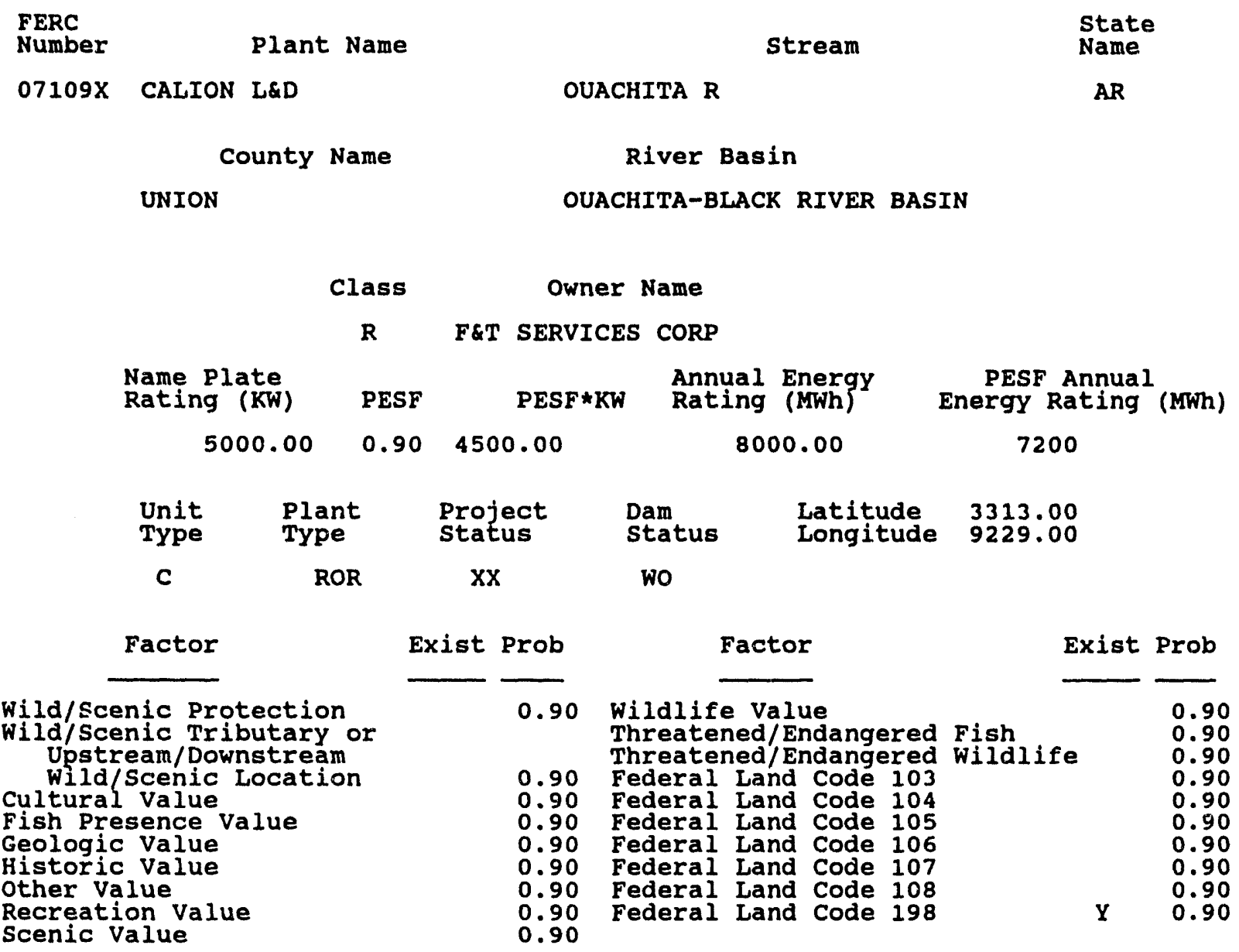


RE S O UR C E D A T A B A S E L I S T I N G

DATE: $10 / 05 / 93$

PAGE NO: 17

FERC

Number

Plant Name

07160X MAMMOTH SPRING

County Name

FULTON

stream

SPRING R, BLACK $\mathbf{R}$

River Basin

LOWER WHITE RIVER BASIN

\section{Class Owner Name}

M AR DEPT OF PARKS \& TOURISM

$\begin{array}{rrrcr}\begin{array}{l}\text { Name Plate } \\ \text { Rating }(K W)\end{array} & \text { PESF } & \text { PESF*KW } & \begin{array}{c}\text { Annual Energy } \\ \text { Rating }(\text { MWh }\end{array} & \begin{array}{c}\text { PESF Annual } \\ \text { EnergY Rating (MWh) }\end{array} \\ 440.00 & 0.50 & 220.00 & 1800.00 & 900\end{array}$

$\begin{array}{cccccc}\text { Unit } & \text { Plant } & \text { Project } & \text { Dam } & \text { Latitude } & 3629.00 \\ \text { Type } & \text { Type } & \text { Status } & \text { Status } & \text { Longitude } & 9132.00 \\ \text { C } & \text { ROR } & \text { XX } & \text { wo } & & \end{array}$

Factor

Wild/Scenic Protection Wild/Scenic Tributary or Upstream/Downstream wild/Scenic Location Cultural value

Fish Presence Value

Geologic Value

Historic Value

other Value

Recreation Value

Scenic Value
Exist Prob

0.90

0.90

0.90

$Y \quad 0.75$

$\mathrm{Y} \quad 0.90$

0.90

0.90

$\mathrm{Y}$
$\mathrm{Y}$
Factor

Wildife Value

Threatened/Endangered Fish

Threatened/Endangered Wildlife

Federal Land Code 104

Federal Land Code 105

Federal Land Code 106

Federal Land Code 107

Federal Land Code 108

Federal Land Code 198
State

Name

AR 
R E S O U R C D A T A B A S E L I S T I N G

DATE: $10 / 05 / 93$

PAGE NO: 18

FERC

Number

Plant Name

stream

state

$07964 X$ DAM 3

SPRING $\mathbf{R}$

Name

AR

County Name

River Basin

FULTON

LOWER WHITE RIVER BASIN

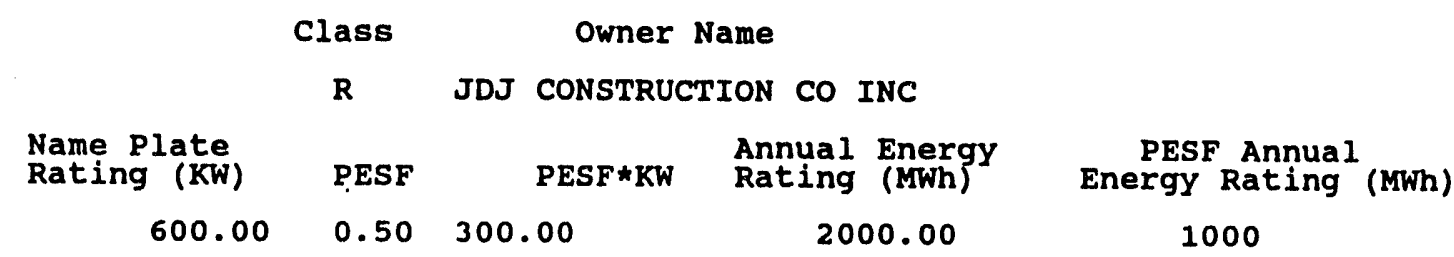

\begin{tabular}{|c|c|c|c|c|c|}
\hline $\begin{array}{l}\text { Unit } \\
\text { Type }\end{array}$ & $\begin{array}{l}\text { Plant } \\
\text { Type }\end{array}$ & $\begin{array}{l}\text { Project } \\
\text { status }\end{array}$ & $\begin{array}{l}\text { Dam } \\
\text { status }\end{array}$ & $\begin{array}{l}\text { Latitude } \\
\text { Longitude }\end{array}$ & $\begin{array}{l}3620.00 \\
9134.00\end{array}$ \\
\hline C & ROR & $X X$ & พอ & & \\
\hline
\end{tabular}

Factor

Exist Prob

Factor

Exist Prob

Wild/Scenic Protection

wild/Scenic Tributary or

Upstream/Downstream

wild/Scenic Location

Cultural value

Fish Presence Value

Geologic value

Historic Value

other Value

\begin{tabular}{cc} 
& \\
\cline { 2 - 2 } & 0.90 \\
& \\
& 0.90 \\
$Y$ & 0.90 \\
$Y$ & 0.75 \\
& 0.90 \\
& 0.90 \\
$Y$ & 0.90 \\
$Y$ & 0.75 \\
$Y$ & 0.90
\end{tabular}

\begin{tabular}{|c|c|c|c|c|}
\hline & Factor & & Exist & Prob \\
\hline $\begin{array}{l}\text { Wildlife } \\
\text { Threater } \\
\text { Threater } \\
\text { Federal } \\
\text { Federal } \\
\text { Federal } \\
\text { Federal } \\
\text { Federal } \\
\text { Federal } \\
\text { Federal }\end{array}$ & $\begin{array}{l}\text { e Value } \\
\text { ned/Endangered } \\
\text { ned/Endangered } \\
\text { Land Code } 103 \\
\text { Land Code } 104 \\
\text { Land Code } 105 \\
\text { Land Code } 106 \\
\text { Land Code } 107 \\
\text { Land Code } 108 \\
\text { Land Code } 198\end{array}$ & $\begin{array}{l}\text { Fish } \\
\text { wildlife }\end{array}$ & $e^{Y}$ & $\begin{array}{l}0.75 \\
0.90 \\
0.90 \\
0.90 \\
0.90 \\
0.90 \\
0.90 \\
0.90 \\
0.90 \\
0.90\end{array}$ \\
\hline
\end{tabular}


RE SOUR C E D A T A B A S E L I S T I N G

DATE: $10 / 05 / 93$

PAGE NO: 19

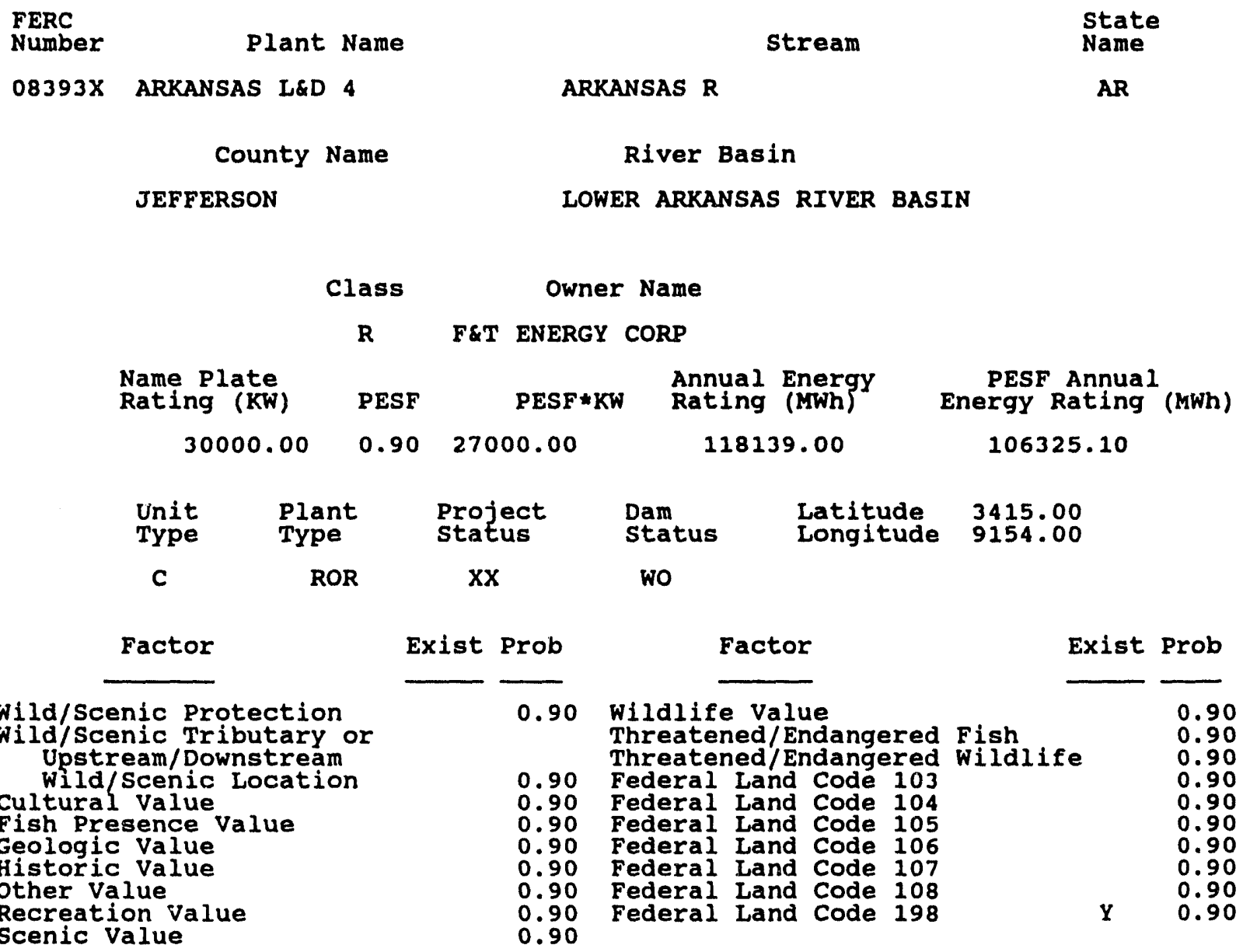


DATE: $10 / 05 / 93$

PAGE NO: 20

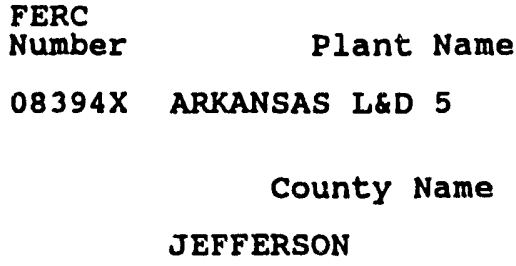

\author{
ARKANSAS $\mathbf{R}$
}

stream

State

Name

AR

$$
\text { River Basin }
$$

LOWER ARKANSAS RIVER BASIN 
R E S O U R C E D A T A B A S E L I S T I N G

DATE: $10 / 05 / 93$

FERC

Number

Plant Name

PAGE NO: 21

$08395 x$

TERRY L\&D 6

County Name

PULASKI

stream

state

Name

ARKANSAS $\mathbf{R}$

AR

River Basin

LOWER ARKANSAS RIVER BASIN

Class Owner Name

R F\&T ENERGY CORP

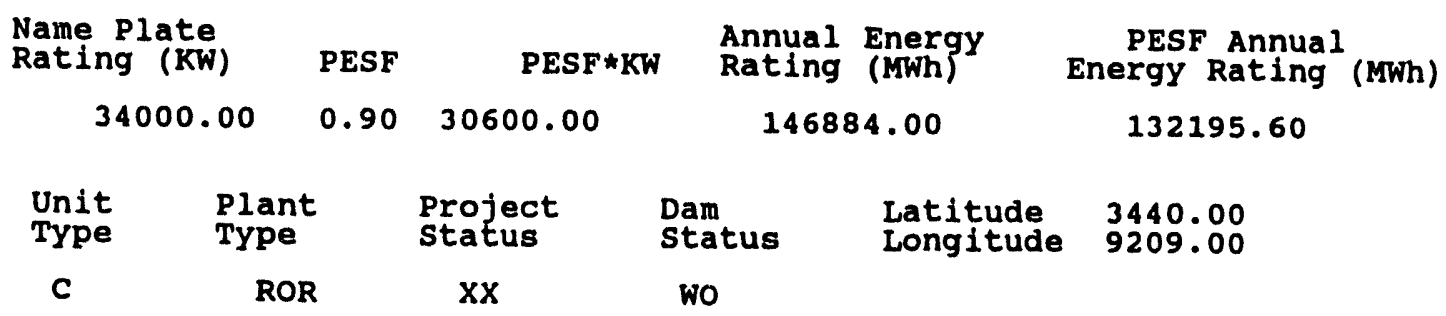

Factor

Exist Prob

Wild/Scenic Protection

wild/Scenic Tributary or Upstream/Downstream wild/Scenic Location Cultural value

Fish Presence Value

Geologic Value

Historic Value

other Value

Recreation Value

Scenic Value

Exist Prob

Factor

0.90

Wildlife Value

Threatened/Endangered $\mathrm{Fish}$

Threatened/Endangered Wildlife

0.90 Federal Land Code 103

0.90 Federal Land Code 104

0.90 Federal Land Code 105

0.90 Federal Land Code 106

0.90 Federal Land Code 107

0.90 Federal Land Code 108

0.90 Federal Land Code 198

0.90

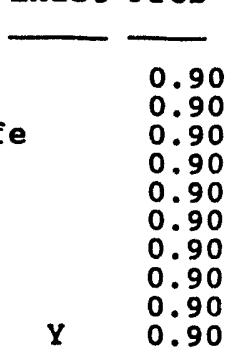


RE SOUR C E DA TABASE I I S T I N G

DATE: $10 / 05 / 93$

PAGE NO: 22

FERC

Number

$09020 x$
Plant Name

HATCHERY CONDUIT

County Name BAXTER stream

NORFORK LAKE, N FK $R$

River Basin

UPPER WHITE RIVER BASIN

\section{Class Owner Name}

$R$ JDJ ENERGY CO

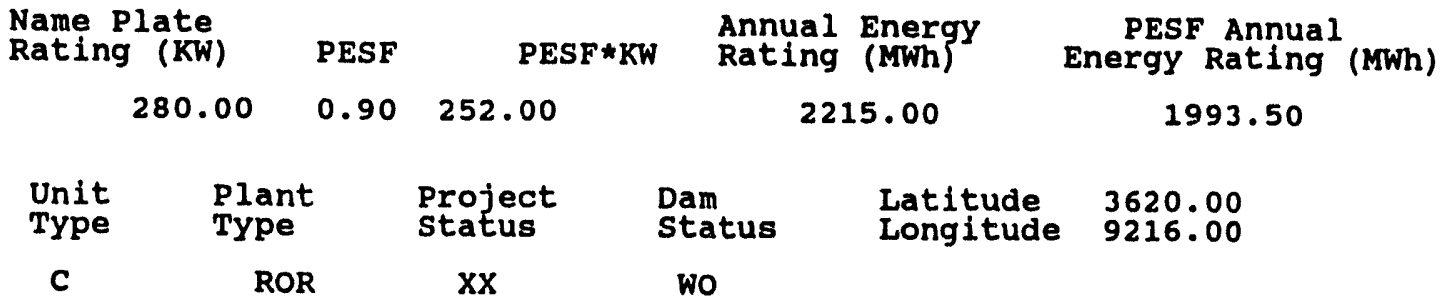

Factor

Exist Prob

Factor

Exist Prob

Wild/Scenic Protection

wild/Scenic Tributary or

Upstream/Downstream

wild/Scenic Location

Cultural value

Fish Presence Value

Geologic Value

Historic Value

other Value

Recreation value

Scenic Value

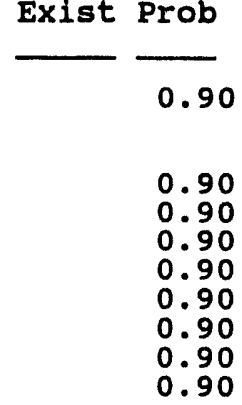


RES OURCE DATABASE L I S T I NG

DATE: $10 / 05 / 93$

PAGE NO: 23

FERC

Number

Plant Name

stream

State

FISH HATCHERY

LITTLE RED R

AR

County Name

River Basin

CLEBURNE

LOWER WHITE RIVER BASIN

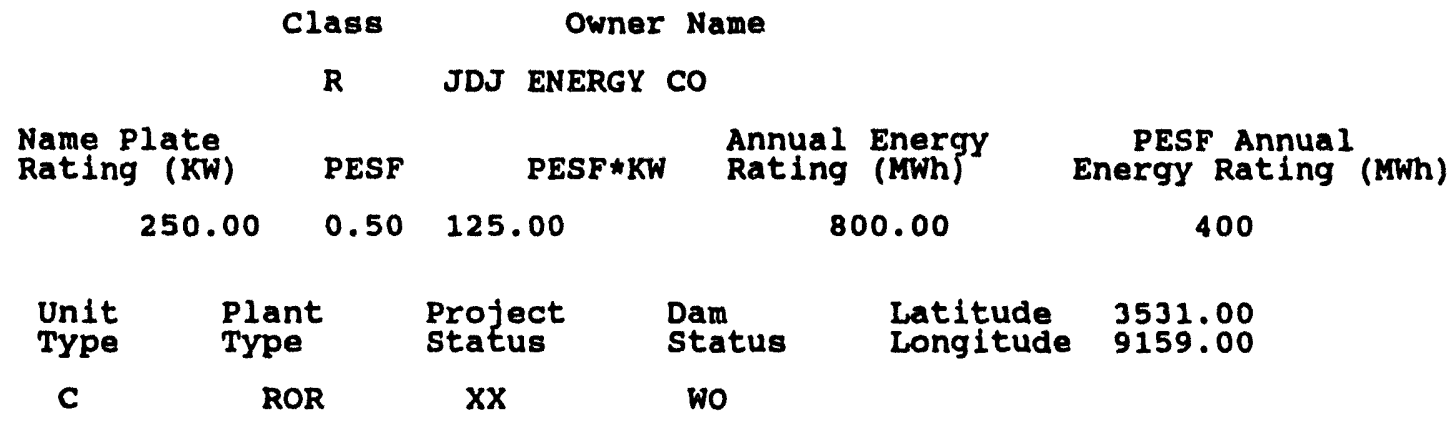

Factor

Exist Prob

Wildife value

Exist Prob

Wild/Scenic Protection

wild/Scenic Tributary or Upstream/Downstream wild/Scenic Location

Cultural value

Fish Presence value

Geologic Value

$\longrightarrow \frac{}{0.90}$

Threatened/Endangered Fish Threatened/Endangered Wildife

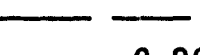

0.90 Federal Land Code 103
0.90 Federal Land Code 104

0.90 Federal Land Code 104

0.90 Federal Land Code 106

0.90 Federal Land Code 107

Historic Value

Other Value

Recreation Value

Scenic Value

Federal Land Code 108

0.90

0.90

0.90

0.90

0.90

0.90

0.90

0.90

0.90

Y 0.90

$\mathbf{Y}$

0.90 
RESOU R E D A T A B A E I I S T I N G

DATE: $10 / 05 / 93$

PAGE NO: 24

FERC

Number Plant Name

$09392 \times$ FELSENTHAL LED

County Name

UNION

stream

State

Name

OUACHITA R

AR

River Basin

OUACHITA-BLACK RIVER BASIN

Class Owner Name

$R$ INDEPENDENCE ELEC CORP

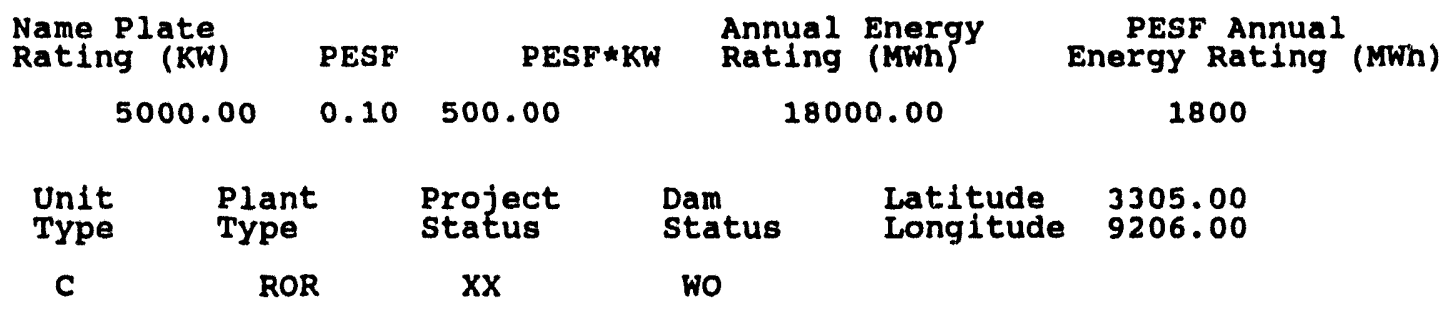

\section{Factor}

Wild/Scenic Protection

wild/Scenic Tributary or Upstream/Downstream wild/Scenic Location Cultural Value

Fish Presence Value

Geologic Value

Historic Value

other value

Recreation Value

Scenic Value
Exist Prob

Factor

Exist Prob

0.90

Wildilfe Value

Threatened/Endangered Fish Threatened/Endangered Wildife

0.90

0.90

0.90

0.90

0.90

0.90

0.90

0.90

Federal Land Code 103

Federal Land Code 104

Federal Land Code 105

Federal Land Code 106

Federal Land Code 107

Federal Land Code 108

Federal Land Code 198

0.90

0.90

0.90

0.90

0.90

$x$

0.10

0.90

0.90

0.90

0.90 
RESOURCE DATABA SE L I S T IN G

DATE: $10 / 05 / 93$

PAGE NO: 25

FERC

Number

Plant Name

stream

State

09440X BLUE MOUNTAIN

PETIT JEAN R

AR

County Name

YELL.

River Basin

LOWER ARKANSAS RIVER BASIN

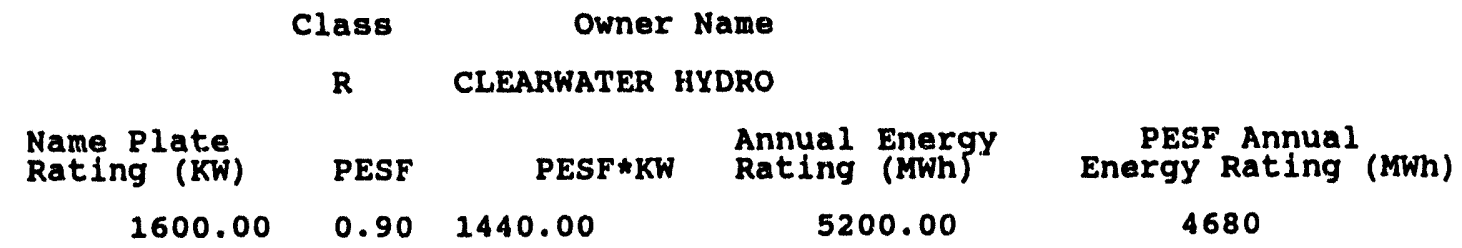

$\begin{array}{cccccc}\begin{array}{c}\text { Unit } \\ \text { Type }\end{array} & \begin{array}{c}\text { Plant } \\ \text { Type }\end{array} & \begin{array}{l}\text { Project } \\ \text { Status }\end{array} & \begin{array}{l}\text { Dam } \\ \text { Status }\end{array} & \begin{array}{l}\text { Latitude } \\ \text { Longitude }\end{array} & \begin{array}{l}3506.00 \\ 939.00\end{array} \\ \text { C } & \text { ROR } & \text { XX } & \text { wo } & & \end{array}$

Factor

Exist Prob

Factor

Exist Prob

wild/Scenic Protection

Wild/Scenic Tributary or

Upstream/Downstream

wild/Scenic Location

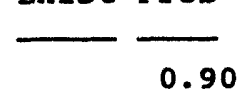

Wildife value

-

Threatened/Endangered Fish

0.90

0.90 Federal Land Code 103

0.90

Cultural Value

Fish Presence Value

Geologic Value

0.90

Federal Land Code 104

0.90 Federal Land Code 105

Federal Land Code 105

0.90 Federal Land Code 106

0.90 Federal Land Code 108

other Value

Federal Land Code 108
Federal Land Code 198

0.90

0.90

0.90

0.90

0.90

0.90

0.90

$\quad 0.90$

Recreation Value

0.90 
RES O URCE DA TA B A S E I I S T I N G

DATE: $10 / 05 / 93$

PAGE NO: 26

FERC

Number

$09607 A$

DEGRAY RRG

County Name

CLARK
Plant Name

CADDO R

$$
\text { River Basin }
$$

OUACHITA-BLACK RIVER BASIN

\begin{tabular}{|c|c|c|c|c|c|}
\hline \multirow[b]{3}{*}{$\begin{array}{l}\text { Name Plate } \\
\text { Rating (KW) }\end{array}$} & $1 \mathbf{a s s}$ & \multicolumn{3}{|c|}{ Owner Name } & \multirow[b]{3}{*}{$\begin{array}{l}\text { PESF Annual } \\
\text { Energy Rating (MWh) }\end{array}$} \\
\hline & $\mathbf{R}$ & JDJ ENERGY & $\mathrm{CO}$ & & \\
\hline & PESF & PESF $\star K W$ & $\begin{array}{l}\text { Annual } \\
\text { Rating }\end{array}$ & $\begin{array}{l}\text { Energy } \\
\text { (NWh) }\end{array}$ & \\
\hline 1040.00 & 0.90 & 936.00 & \multicolumn{2}{|c|}{4436.00} & 3992.40 \\
\hline $\begin{array}{l}\text { Unit } \\
\text { Type }\end{array}$ & & $\begin{array}{l}\text { Project } \\
\text { status }\end{array}$ & $\begin{array}{l}\text { Dam } \\
\text { status }\end{array}$ & $\begin{array}{l}\text { Latitude } \\
\text { Longitude }\end{array}$ & $\begin{array}{l}3411.00 \\
9305.00\end{array}$ \\
\hline C & & NA & พอ & & \\
\hline
\end{tabular}

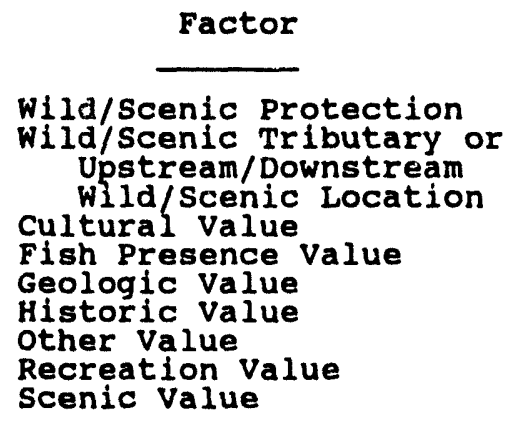

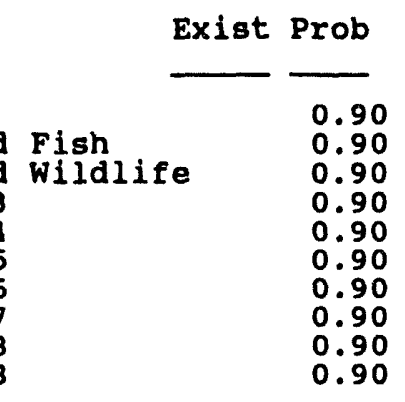


DATE: $10 / 05 / 93$

PAGE NO: 27

FERC

Number

Plant Name

$10248 X$ TOAD SUCK L\&D 8

County Name

PERRY state

Name

ARKANSAS $\mathbf{R}$

$A R$

River Basin

LOWER ARKANSAS RIVER BASIN

\section{Class Owner Name}

R F\&T SERVICES CORP

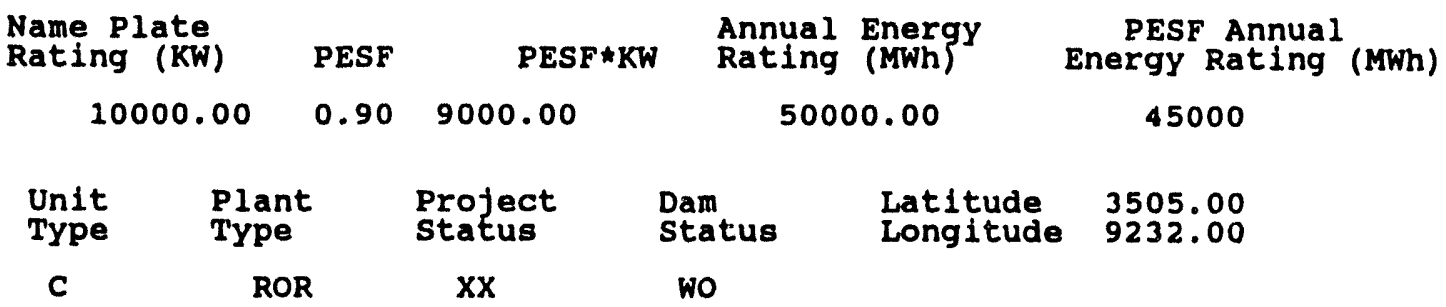

Factor

Exist Prob

Factor

Exist Prob

Wild/Scenic Protection

wild/Scenic Tributary or

Upstream/Downstream

wild/Scenic Location

Cultural value

Fish Presence Value

Geologic Value

Historic Value

other Value

Recreation value

Scenic Value

\begin{tabular}{c} 
- \\
\hline 0.90 \\
0.90 \\
0.90 \\
0.90 \\
0.90 \\
0.90 \\
0.90 \\
0.90 \\
0.90
\end{tabular}

Wildife Value

Threatened/Endangered $\mathrm{F}$ ish

Threatened/Endangered Wildife

Federal Land Code 103

Federal Land Code 104

Federal Land Code 105

Federal Land Code 106

Federal Land Code 107

Federal Land Code 108

Federal Land Code 198

0.90

0.90

0.90

0.90

0.90

0.90

0.90

0.90

0.90

$Y$

0.90 
RESOURCE D A T A B A S E L I S T I N G

DATE: $10 / 05 / 93$

PAGE NO: 28

FERC

Number Plant Name

10250X DE QUEEN

State

\title{
$10250 x$
}

DE QUEEN

County Name SEVIER

\author{
Stream \\ ROLLING FK, LITTLE R \\ River Basin \\ LOWER RED RIVER BASIN
}

Name

AR

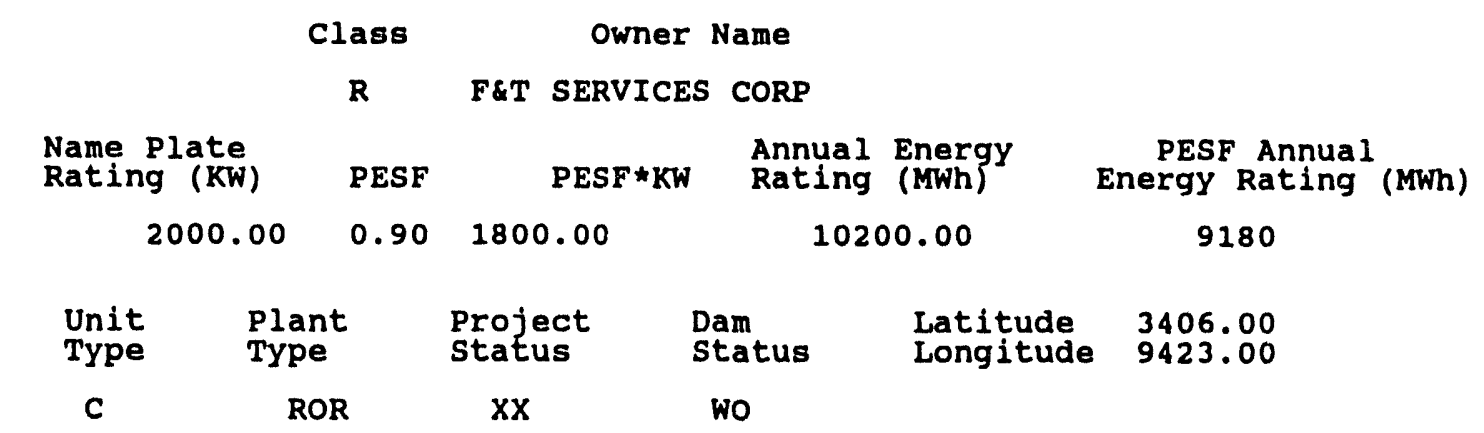

Factor

\begin{tabular}{l} 
Exist Prob \\
\hline 0.00 \\
\\
0.00 \\
0.00 \\
0.00 \\
0.00 \\
0.00 \\
0.00 \\
0.00 \\
0.00
\end{tabular}

\begin{tabular}{|c|c|c|c|}
\hline & Factor & Exist & Prob \\
\hline $\begin{array}{l}\text { Wildiif } \\
\text { Threate } \\
\text { Threatel } \\
\text { Federal } \\
\text { Federal } \\
\text { Federal } \\
\text { Federal } \\
\text { Federal } \\
\text { Federal } \\
\text { Federal }\end{array}$ & $\begin{array}{l}\text { e Value } \\
\text { ned/Endangered } \\
\text { ned/Endangered } \\
\text { Land Code } 103 \\
\text { Land Code } 104 \\
\text { Land Code } 105 \\
\text { Land Code } 106 \\
\text { Land Code } 107 \\
\text { Land Code } 108 \\
\text { Land Code } 198\end{array}$ & $\begin{array}{l}\text { Fish } \\
\text { wildlife }\end{array}$ & $\begin{array}{l}0.00 \\
0.00 \\
0.00 \\
0.90 \\
0.90 \\
0.90 \\
0.90 \\
0.90 \\
0.90 \\
0.90\end{array}$ \\
\hline
\end{tabular}

Wild/Scenic Protection

Wild/Scenic Tributary or

Upstream/ Downstream

wild/scenic Location

Cultural. Value

Fish Presence Value

Geologic Value

Historic Value

other Value

Recreation Value

Scenic Value

.00

0.00

0.90

0.90

0.90

0.90

Scenic Value 
RESOURCE DATABASE L I S T ING

DATE: $10 / 05 / 93$

PAGE NO: 29

FERC

Number

Plant Name

stream

State

$10251 X$

DIERKS

SALINE R

AR

County Name

River Basin

SEVIER

LOWER RED RIVER BASIN

\begin{tabular}{|c|c|c|c|c|c|c|c|}
\hline \multirow{3}{*}{\multicolumn{2}{|c|}{$\begin{array}{l}\text { Name Plate } \\
\text { Rating (KW) }\end{array}$}} & & \multicolumn{3}{|c|}{ Owner Name } & & \multirow[b]{3}{*}{$\begin{array}{l}\text { PESF Annual } \\
\text { Energy Rating }\end{array}$} \\
\hline & & & FET & SERVICES & CORP & & \\
\hline & & PESF & & $P E S F \star K W$ & $\begin{array}{l}\text { Annual } \\
\text { Rating }\end{array}$ & $\begin{array}{l}\text { Energy } \\
(\text { MWh })\end{array}$ & \\
\hline \multicolumn{2}{|c|}{2000.00} & 0.90 & \multicolumn{2}{|c|}{1800.00} & \multicolumn{2}{|c|}{10200.00} & 9180 \\
\hline $\begin{array}{l}\text { Unit } \\
\text { Type }\end{array}$ & \multicolumn{2}{|c|}{$\begin{array}{l}\text { Plant } \\
\text { Type }\end{array}$} & \multicolumn{2}{|c|}{$\begin{array}{l}\text { Project } \\
\text { status }\end{array}$} & $\begin{array}{l}\text { Dam } \\
\text { Status }\end{array}$ & $\begin{array}{l}\text { Latitude } \\
\text { Longitude }\end{array}$ & $\begin{array}{l}3408.00 \\
9406.00\end{array}$ \\
\hline C & \multicolumn{2}{|c|}{ ROR } & $\mathbf{x x}$ & & ro & & \\
\hline
\end{tabular}

Factor

Exist Prob

Factor

Exist Prob

wild/Scenic Protection

wild/Scenic Tributary or

Upstream/Downstream

wild/Scenic Location

\begin{tabular}{c}
0.90 \\
\hline 0.90 \\
0.90 \\
0.90 \\
0.90 \\
0.90 \\
0.90 \\
0.90 \\
0.90
\end{tabular}

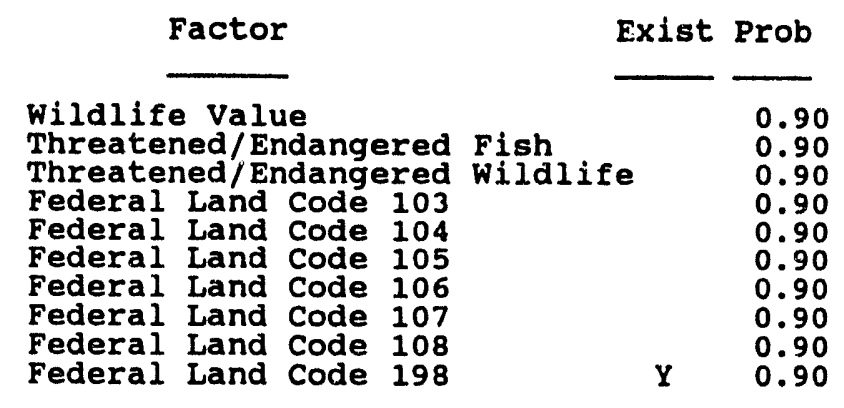

Cultural Value

Fish Presence Value

Genlogic Value

Historic Value

Other value

Recreation value

Scenj I Value

0.90

.90

0.90

.90

0.90
0.90 
R E S O U R C E D A T A B A S E L I S T I N G

DATE : $10 / 05 / 93$

PAGE NO: 30

FERC

Number

Plant Name

stream

State

10546A MILLWOOD

LITTLE $R$

AR

County Name

River Basin

HEMPSTEAD

LOWER RED RIVER BASIN

\begin{tabular}{|c|c|c|c|c|c|}
\hline \multirow[b]{3}{*}{$\begin{array}{l}\text { Name Plate } \\
\text { Rating (KW) }\end{array}$} & \multicolumn{4}{|c|}{ Owner Name } & \\
\hline & $\mathbf{R}$ & MILLWOOD HYD & ASSOC & & \\
\hline & PESF & $P E S F \star K W$ & $\begin{array}{l}\text { Annual } \\
\text { Rating }\end{array}$ & $\begin{array}{l}\text { Energy } \\
\text { (MWh) }\end{array}$ & $\begin{array}{l}\text { PESF Annual } \\
\text { Energy Rating (MWh) }\end{array}$ \\
\hline 15000.00 & 0.90 & 13500.00 & 8500 & 0.00 & 76500 \\
\hline
\end{tabular}

$\begin{array}{cccccc}\text { Unit } & \begin{array}{l}\text { Plant } \\ \text { Type }\end{array} & \begin{array}{l}\text { Project } \\ \text { Status }\end{array} & \begin{array}{l}\text { Dam } \\ \text { Status }\end{array} & \begin{array}{l}\text { Latitude } \\ \text { Longitude }\end{array} & \mathbf{3 3 4 2 . 0 0} 9358.00 \\ \text { C } & \text { ROR } & \text { Po } & \text { wo } & & \end{array}$

Factor

Exist Prob

Factor

Exist Prob

Wild/Scenic Protection

Wild/Scenic Tributary or

Upstream/Downstream

wild/Scenic Location

Cultural value

Fish Presence Value

Geologic Value

Historic Value

other Value

Recreation value

scenic value

0.90


0.90
0.90
0.90
0.90
0.90
0.90
0.90
0.90

\begin{tabular}{|c|c|c|c|c|c|}
\hline & Factor & & & Exist & Prob \\
\hline $\begin{array}{l}\text { Wildlif } \\
\text { Threate } \\
\text { Threate } \\
\text { Federal } \\
\text { Federal } \\
\text { Federal } \\
\text { Federal } \\
\text { Federal } \\
\text { Federal }\end{array}$ & $\begin{array}{l}\text { Ee Valu } \\
\text { ned/En } \\
\text { ened/En } \\
\text { Land } \\
\text { Land } \\
\text { Land } \\
\text { Land } \\
\text { Land } \\
\text { Land } \\
\text { Land }\end{array}$ & $\begin{array}{l}\text { le } \\
\text { dangered } \\
\text { dangered } \\
\text { Code } 103 \\
\text { Code } 104 \\
\text { Code } 105 \\
\text { Code } 106 \\
\text { Code } 107 \\
\text { Code } 108 \\
\text { Code } 198\end{array}$ & $\begin{array}{l}\text { Fish } \\
\text { wildlife }\end{array}$ & e & $\begin{array}{l}0.90 \\
0.90 \\
0.90 \\
0.90 \\
0.90 \\
0.90 \\
0.90 \\
0.90 \\
0.90 \\
0.90\end{array}$ \\
\hline
\end{tabular}


RE S O U R C E D A T A B A S E I I S T I N G

DATE: $10 / 05 / 93$

PAGE NO: 31

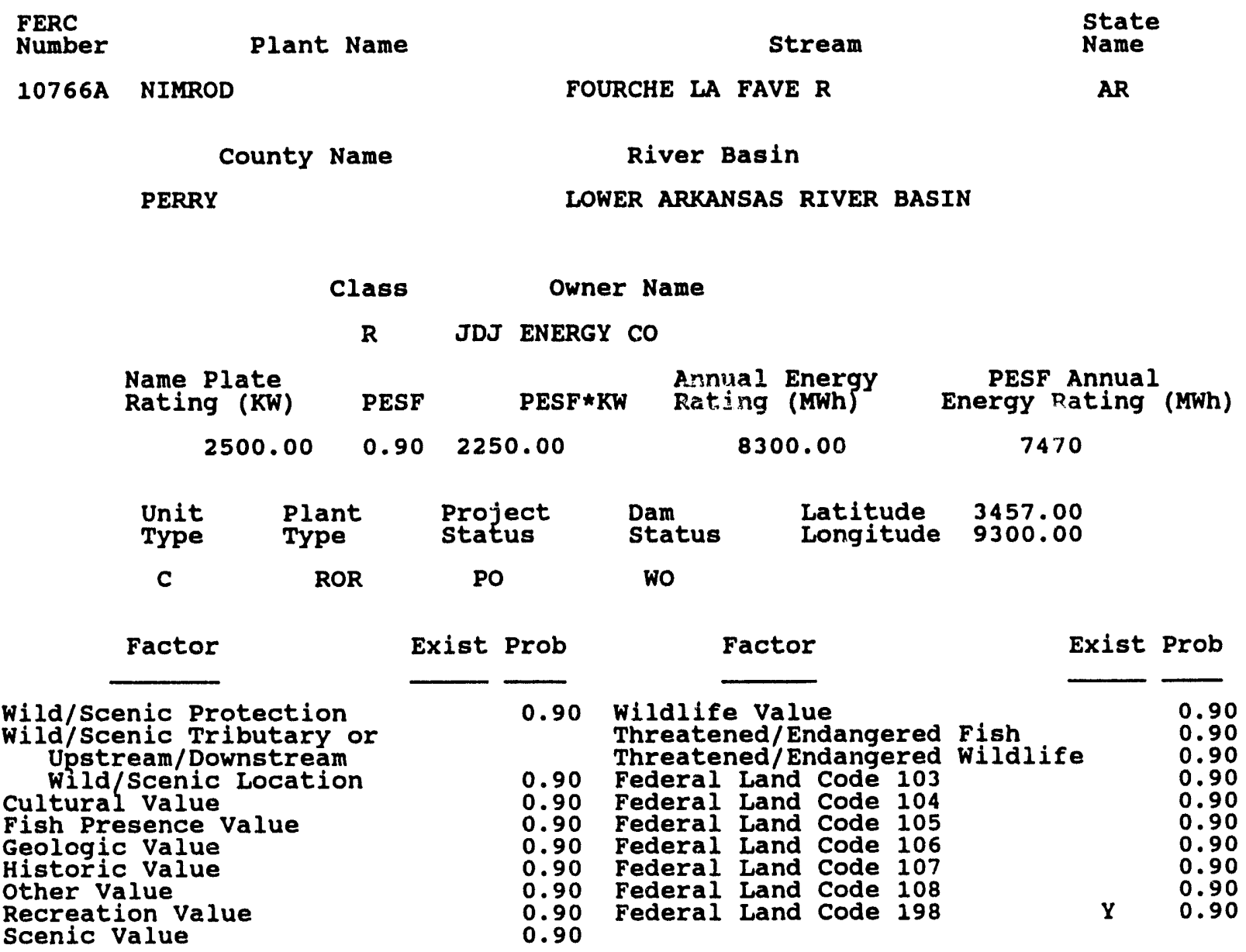


DATE: $10 / 05 / 93$

PAGE NO: 32

FERC

Number

Plant Name

AR001 KIRKLAND

County Name

OUACHITA stream

SMACKOVER CR, OUACHITA R

River Basin

OUACHITA-BLACK RIVER BASIN
Class

Owner Name

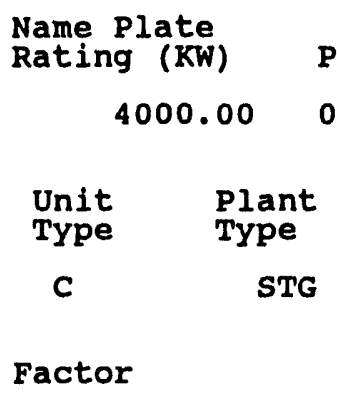

STG

C

Factor

wild/Scenic Protection

wild/Scenic Tributary or

Upstream/Downstream wild/Scenic Location cultural value

Fish Presence Value

Geologic Value

Historic Value

other Value

Recreation Value

Scenic Value
Exist Prob

0.90

Wildiffe Value Threatened/Endangered Fish Threatened/Endangered Wildlife

0.90

0.90

Federal Land Code 103

Federal Land Code 104

Federal Land Code 105

0.90 Federal Land Code 106
0.90 Federal Land Code 107

0.90 Federal Land Code 108

0.90 Federal Land Code 198
PESF Annual Energy Rating (MWh) 5400

0.00

0.00
Name

AR

Exist Prob

0.90

0.90

0.90

0.90

0.90

0.90

0.90

0.90

0.90

0.90 
DATE: $10 / 05 / 93$

PAGE NO: 33

FERC

Number

Plant Name

stream

State

$\mathrm{AROO2}$

CADDO GAP

CADDO $R$

Name

County Name

River Basin

MONTGOMERY

OUACHITA-BLACK RIVER BASIN

Class

Owner Name

\begin{tabular}{|c|c|c|c|c|c|c|}
\hline $\begin{array}{l}\text { Name P } \\
\text { Rating }\end{array}$ & & PESF & $\mathrm{PESF} \star \mathrm{KK}$ & $\begin{array}{l}\text { Annt } \\
\text { Rat }\end{array}$ & $\begin{array}{l}\text { Energy } \\
\text { (MWh) }\end{array}$ & $\begin{array}{l}\text { PESF Annual } \\
\text { Energy Rating (MWh) }\end{array}$ \\
\hline & .00 & 0.50 & 1150.00 & & 00.00 & 4350 \\
\hline $\begin{array}{l}\text { Unit } \\
\text { Type }\end{array}$ & $\begin{array}{l}\text { Pl } \\
\text { Tyl }\end{array}$ & & $\begin{array}{l}\text { Project } \\
\text { Status }\end{array}$ & $\begin{array}{l}\text { Dam } \\
\text { status }\end{array}$ & $\begin{array}{l}\text { Latitude } \\
\text { Longitude }\end{array}$ & $\begin{array}{l}3429.00 \\
9338.00\end{array}$ \\
\hline C & & & ZZ & $\mathbf{U}$ & & \\
\hline
\end{tabular}

Factor

Exist Prob

Factor

Exist Prob

Wild/Scenic Protection

wild/Scenic Tributary or

Upstream/Downstream

Wild/Scenic Location

Cultural value

Fish Presence Value

Geologic Value

Historic Value

other Value

Recreation Value

Scenic Value

\begin{tabular}{c} 
Exist Prob \\
\\
\hline 0.90 \\
0.90 \\
0.90 \\
0.90 \\
0.90 \\
0.90 \\
0.90 \\
0.90 \\
0.90
\end{tabular}

\begin{tabular}{|c|c|c|c|}
\hline & Factor & Exist & Prob \\
\hline $\begin{array}{l}\text { Wildlif } \\
\text { Threate } \\
\text { Threate } \\
\text { Federal } \\
\text { Federal } \\
\text { Federal } \\
\text { Federal } \\
\text { Federal } \\
\text { Federal } \\
\text { Federal }\end{array}$ & $\begin{array}{l}\text { Value } \\
\text { ened/Endangered } \\
\text { ened/Endangered } \\
\text { Land Code } 103 \\
\text { Land Code } 104 \\
\text { Land Code } 105 \\
\text { Land Code } 106 \\
\text { Land Code } 107 \\
\text { Land Code } 108 \\
\text { Land Code } 198\end{array}$ & $\begin{array}{l}\text { Fish } \\
\text { wildilife }\end{array}$ & $\begin{array}{l}0.90 \\
0.90 \\
0.90 \\
0.90 \\
0.50 \\
0.90 \\
0.90 \\
0.90 \\
0.90 \\
0.90\end{array}$ \\
\hline
\end{tabular}


RE S O U R C E D A T A B A S E I I S T I N G

DATE: $10 / 05 / 93$

PAGE NO: 34

FERC

Number

Plant Name

AR003 ROCKPORT

County Name

HOT SPRING

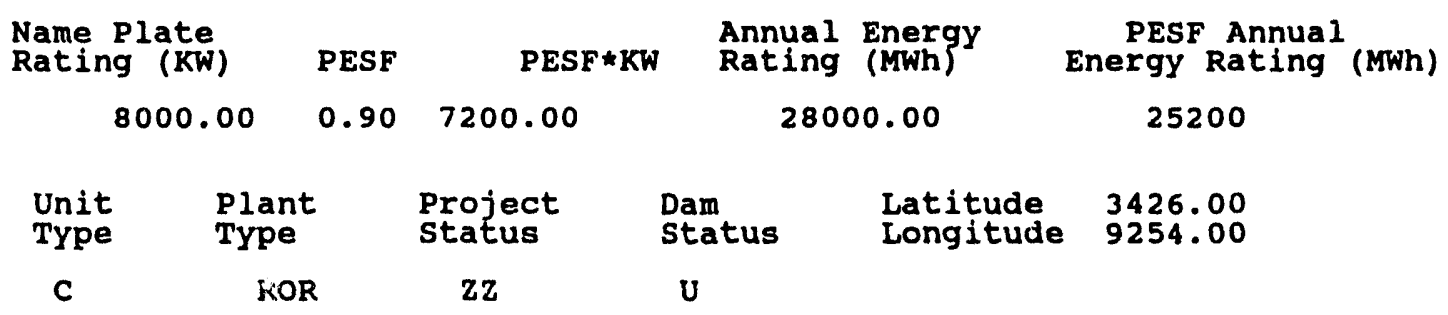

\section{Factor}

Wild/Scenic protection

wild/scenic Tributary or Upstream/Downstream wild/Scenic Location

Cultural Value

Fish Presence Value

Geologic Value

Historic Value

other Value

Recreation value

Scenic Value stream

OUACHITA $R$

River Basin

OUACHITA-BLACK RIVER BASIN
Class

Owner Name

\begin{tabular}{c} 
Exist Prob \\
\hline 0.90 \\
0.90 \\
0.90 \\
0.90 \\
0.90 \\
0.90 \\
0.90 \\
0.90 \\
0.90
\end{tabular}

Factor

Exist Prob

Wildlife Value

Threatened/Endangered Fish

Threatened/Endangered Wildlife

State

Name

AR

Federal Land Code 104

Federal Land Code 105

Federal Land Code 106

Federal Land Code 107

Federal Land Code 108

Federal Land Code 198

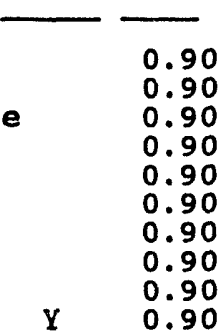


RE S O UR C E D A T A B A S E L I S T I N G

DATE: $10 / 05 / 93$

PAGE NO: 35

FERC

Number

AR004

FIDDLERS CREEK

County Name

MONTGOMERY

Class

Owner Name

Name Plate
Rating (KW)

20000.00

PESF

PESF

PESF *KW

0.102000 .00

Annual Energy

Rating (KWh)

River Basin

OUACHITA-BLACK RIVER BASIN
State

Name

AR

\section{Unit \\ Type

\begin{abstract}
Plant
\end{abstract}
c

Factor

Wild/Scenic Protection wild/Scenic Tributary or Upstream/Downstream wild/scenic Location Cultural Value

Fish Presence Value Geologic Value

Historic Value

other Value

Recreation value

Scenic Value
ROR

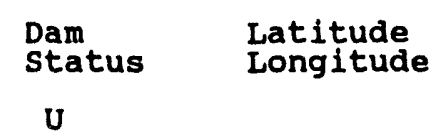

Factor

Exist Prob

$\begin{array}{cc} & \\ & 0.90 \\ & \\ & 0.90 \\ & 0.90 \\ Y & 0.25 \\ Y & 0.50 \\ & 0.90 \\ Y & 0.90 \\ Y & 0.25 \\ & 0.50\end{array}$

PESF Annual Energy Rating (MWh)

\section{0}

3439.00

9356.00

Factor
wildlife Value
Threatened/Endangered Fish
Threatened/Endangered wildlife
Federal Land Code 103
Federal Land Code 104
Federal Land Code 105
Federal Land Code 106
Federal Land Code 107
Federal Land Code 108
Federal Land Code 198

Exist Prob

0.90

0.90

0.90

$\mathbf{Y}$

0.50

0.90

0.90

0.90

0.90

0.90 

R E S O U R C E
D A T A B A S E
L I $\mathbf{S}$ T I N G

DATE: $10 / 05 / 93$

PAGE NO: 36

FERC

Number

Plant Name

stream

state

AR005

DARDANELLE

ARKANSAS $\mathbf{R}$

Name

County Name

River Basin

YELL

LOWER ARKANSAS RIVER BASIN

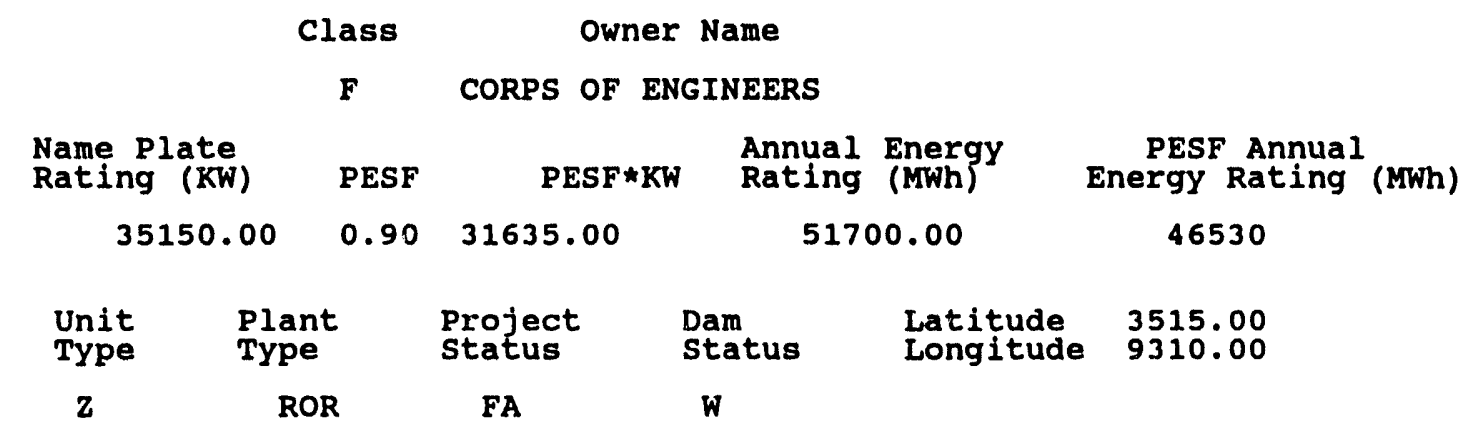

Factor

Exist Prob

Factor

Exist Prob

Wild/scenic Protection

wild/Scenic Tributary or Upstream/Downstream wild/Scenic Location

Cultural value

Fish Presence Value

Geologic Value

Historic Value

Other Value

Recreation Value

Scenic Value

0.90 Wildlife Value

Threatened/Endangered Fish

Threatened/Endangered Wildlife

-

0.90

0.90

0.90 Federal Land Code 103

0.90 Federal Land Code 104

0.90 Federal Land Code 105

0.90 Federal Land Code 106

0.90 Federal Land Code 107

0.90 Federal Land Code 108

0.90 Federal Land Code 198

0.90

0.90

0.90

0.90

0.90

0.90

$\mathbf{Y}$ 
RES O UR C E DA TABASE I I S T I N G

DATE: $10 / 05 / 93$

PAGE NO: 37

FERC

Number

Plant Name

stream

State

AR006

BUFFALO CITY

WHITE $R$

AR

County Name

River Basin

BAXTER

UPPER WHITE RIVER BASIN

Class Owner Name

NHPS/COE

\begin{tabular}{|c|c|c|c|c|}
\hline $\begin{array}{l}\text { Name plate } \\
\text { Rating (KW) }\end{array}$ & PESF & $P E S F \star K W$ & $\begin{array}{l}\text { Annual Energy } \\
\text { Rating (MWh) }\end{array}$ & $\begin{array}{l}\text { PESF Annual } \\
\text { Energy Rating }(\mathrm{MWh})\end{array}$ \\
\hline 55900.00 & 0.90 & 50310.00 & 148000.00 & 133200 \\
\hline
\end{tabular}

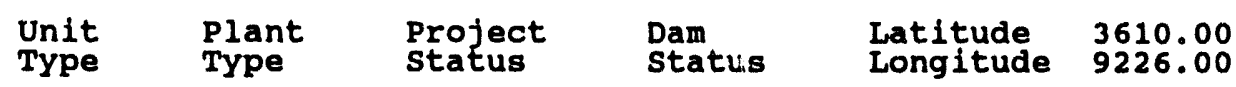

STG

zz

U

Factor

Exist Prob

Factor

Exist Prob

wild/Scenic Protection

Wild/Scenic Tributary or

Upstream/Downstream

wild/Scenic Location

Cultural value

Fish Presence Value

Geologic Value

Historic Value

other Value

Recreation value

Scenic Value

Exist

0.90

wildlife Value

Threatened/Endangered Fish

Threatened/Endangered Wildilfe

0.90 Federal Land Code 103

0.90 Federal Land Code 104

0.90 Federal Land Code 105

0.90 Federal Land Code 106

0.90 Federal Land Code 107

0.90 Federal Land Code 108

0.90 Federal Land Code 198

$\begin{array}{cc} & \\ & \\ & 0.90 \\ & 0.90 \\ & 0.90 \\ & 0.90 \\ 0.90 \\ 0.90 \\ & 0.90 \\ & 0.90 \\ & 0.90 \\ & 0.90\end{array}$



R E S O U R C E
D A T A B A $\mathbf{S}$ E
L I S T I N G

DATE: $10 / 05 / 93$

PAGE NO: 38

FERC

Number

Plant Name

stream

State

AR007 RUSSELVILLE

ARKANSAS $\mathbf{R}$

AR

County Name

River Basin

YELL

LOWER ARKANSAS RIVER BASIN

Class Owner Name

P ARKANSAS PWR AND LT CO

\begin{tabular}{|c|c|c|c|c|c|c|}
\hline \multicolumn{2}{|c|}{$\begin{array}{l}\text { Name Plate } \\
\text { Rating (KW) }\end{array}$} & PESF & PESF $\star K W$ & $\begin{array}{l}\text { Annual } \\
\text { Rating }\end{array}$ & $\begin{array}{l}\text { Energy } \\
\text { (MWh) }\end{array}$ & $\begin{array}{l}\text { PESF An } \\
\text { Energy Rat }\end{array}$ \\
\hline & 0.00 & 0.90 & 0.00 & & 0.00 & \\
\hline $\begin{array}{l}\text { Unit } \\
\text { Type }\end{array}$ & $\begin{array}{l}\text { Pla } \\
\text { Typ }\end{array}$ & & $\begin{array}{l}\text { Project } \\
\text { Status }\end{array}$ & $\begin{array}{l}\text { Dam } \\
\text { status }\end{array}$ & $\begin{array}{l}\text { Latitude } \\
\text { Longitude }\end{array}$ & $\begin{array}{l}0.00 \\
0.00\end{array}$ \\
\hline $\mathbf{z}$ & & & $\mathbf{z Z}$ & พо & & \\
\hline
\end{tabular}

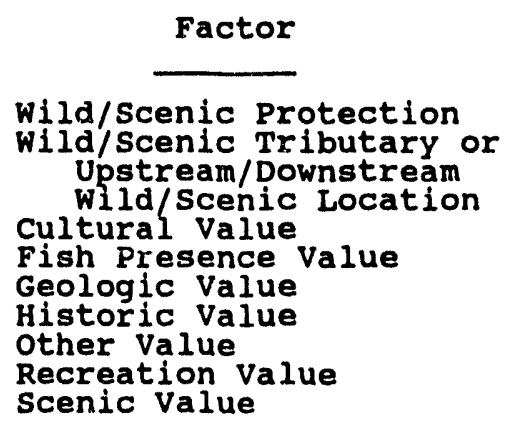

Exist Prob

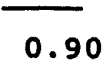

0.90

0.90

0.90

0.90

0.90

0.90

\begin{tabular}{lll}
\multicolumn{1}{c}{ Factor } & Exist & Prob \\
Wildlife Value & \\
Threatened/Endangered Fish & 0.90 \\
Threatened/Endangered wildlife & 0.90 \\
Federal Land Code 103 & 0.90 \\
Federal Land Code 104 & 0.90 \\
Federal Land Code 105 & 0.90 \\
Federal Land Code 106 & 0.90 \\
Federal Land Code 107 & 0.90 \\
Federal Land Code 108 & 0.90 \\
Federal Land Code 198 & 0.90 \\
& & 0.90
\end{tabular}


RE SOU R C E DA TABASE L I S T I N G

DATE: $10 / 05 / 93$

PAGE NO: 39

FERC

Number

Plant Name

AR008

NARROWS

stream

state

Name

LITTLE MISSOURI $R$

AR

County Name

River Basin

PIKE

OUACHITA-BLACK RIVER BASIN

Class Owner Name

$F$ CORPS OF ENGINEERS

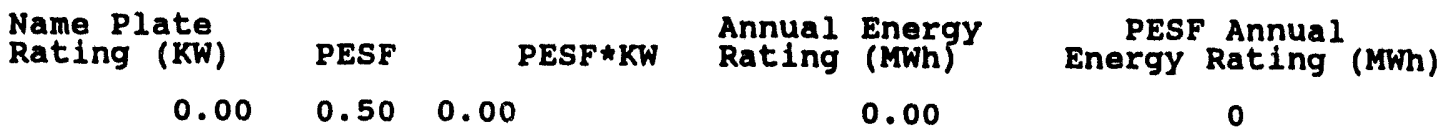

Unit Plant Project Dam Latitude 3409.00

Type Type status status iongitude 9343.00

Z STG FA

พ

Factor

Exist Prob

Factor

Exist Prob

Wild/Scenic Protection

wild/Scenic Tributary or

Upstream/Downstream

Wild/Scenic Location

$\begin{array}{cc} & \\ & 0.90 \\ & \\ & 0.90 \\ & 0.90 \\ Y & 0.75 \\ Y & 0.90 \\ & 0.90 \\ & 0.90 \\ Y & 0.75 \\ Y & 0.90\end{array}$

Factor
Wildife Value
Threatened/Endangered
Threatened/Endangered
Federal Land Code 103
Federal Land Code 104
Federal Land Code 105
Federal Land Code 106
Federal Land Code 107
Federal Land Code 108
Federal Land Code 198

\begin{tabular}{|c|c|c|}
\hline $\begin{array}{l}\text { Fish } \\
\text { Wildilfe }\end{array}$ & $\mathbf{Y}$ & $\begin{array}{l}0.75 \\
0.90 \\
0.90 \\
0.90 \\
0.90 \\
0.90 \\
0.90 \\
0.90 \\
0.90 \\
0.90\end{array}$ \\
\hline
\end{tabular}

Fish Presence Value

Geologic Value

Historic Value

ther Value

Recreation Value

Scenic Value 

R E S O U R C E
D A T A B A E
L I S T I N G

DATE: $10 / 05 / 93$

FERC

Number

Plant Name

PAGE NO: 40

AR009

BLAKELY MOUNTAIN

County Name

GARIAAND

Stream
OUACHITA $R$
RIVer Basin
OUACHITA-BLACK RIVER BASIN

$\begin{array}{cr}\text { Class } & \text { Owner Name } \\ \text { F } & \text { CORPS OF ENGINEERS }\end{array}$

Name plate PESF

Annual Energy Rating (KW)

PESF

PESF $\star K W$

Rating (MWh)

PESF Annual

0.00

0.75

0.00

0.00

Energy Rating (MWh)

Unit

Plant
Type

Project

status

Dam

status

Latitude

Longitude 9312.00

z

STG

FA

พ

Factor

Exist Prob

Factor

Exist Prob

wild/Scenic Protection

Wild/Scenic Tributary or

Upstream/Downstream wild/Scenic Location

Cultural Value

Fish Presence Value

Geologic Value

Historic Value

other value

Recreation Value

scenic value

\begin{tabular}{c} 
\\
\\
\hline 0.90 \\
0.90 \\
0.90 \\
0.90 \\
0.90 \\
0.90 \\
0.90 \\
0.90 \\
0.90
\end{tabular}

wildife Value

Threatened/Endangered Fish

Threatened/Endangered wildife

0

Federal Land Code 103

Federal Land Code 104

Federal Land Code 105

Federal Land Code 106

Federal Land Code 107

Federal Land Code 108

Federal Land Code 198

Name

AR

0.90 
RESOUR C E D A T A B A S E L I S T IN

DATE: $10 / 05 / 93$

PAGE NO: 41

FERC

Number

Plant Name

AR010 GRANDVIEW

County Name

CARROLL

\section{stream}

KINGS R

River Basin

UPPER WHITE RIVER BASIN
State

Name

AR

Class Owner Name

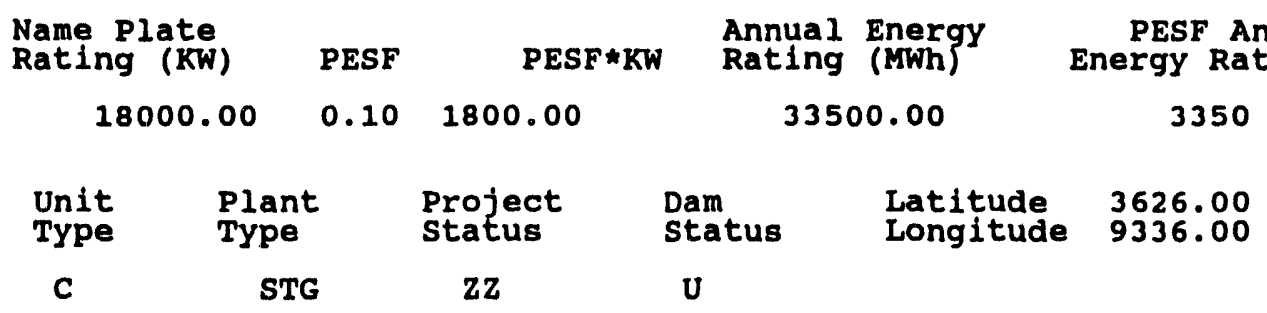

Factor

Exist Prob

Factor

Exist Prob

wild/Scenic Protection

wild/scenic Tributary or

Upstream/Downstream

wild/Scenic Location

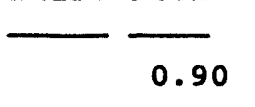

Wildlife Value
Threatened/Endangered
Threatened/Endangered
Federal Land Code 103
Federal Land Code 104
Federal Land Code 105
Federal Land Code 106
Federal Land Code 107
Federal Land Code 108
Federal Land Code 198

\begin{tabular}{|c|c|c|}
\hline $\begin{array}{l}\text { Fish } \\
\text { wildilfe }\end{array}$ & $Y$ & $\begin{array}{l}0.25 \\
0.90 \\
0.90 \\
0.90 \\
0.90 \\
0.90 \\
0.90 \\
0.90 \\
0.90 \\
0.90\end{array}$ \\
\hline
\end{tabular}

Fish presence value

Geologic value

Historic value

other value

Recreation Value

Scenic Value

$\begin{array}{ll} & 0.90 \\ & 0.90 \\ Y & 0.25 \\ Y & 0.50 \\ & 0.90 \\ & 0.90 \\ Y & 0.25 \\ Y & 0.50\end{array}$


RES O UR C E D A T A B A S E L I S T I N G

DATE: $10 / 05 / 93$

PAGE NO: 42

FERC

Number

Plant Name

stream

state

AR011

BEAVER

WHITE $\mathbf{R}$

AR

county Name

River Basin

CARROLL

UPPER WHITE RIVER BASIN

$\begin{array}{cr}\text { Class } & \text { Owner Name } \\ F & \text { CORPS OF ENGINEERS }\end{array}$

Name plate

Annual Energy

Rating (KW)

PESF

$P E S F \star K W$

Rating

(MWh)

PESF Annual

0.00

$0.90 \quad 0.00$

Energy Rating (MWh)

Unit

Type

Plant
Type
STG

Z

Factor

Exist Prob

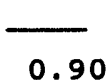

wild/Scenic Protection

Wild/Scenic Tributary or

Upstream/Downstream

wild/Scenic Location

Cultural value

Fish Presence Value

Geologic Value

Historic value

other Value

Recreation Value

Scenic Value

$$
\begin{aligned}
& \text { Project } \\
& \text { status }
\end{aligned}
$$

FA
Dam
status
W

Latitude

Longitude

3625.00
0.00

\begin{tabular}{|c|c|c|c|}
\hline & Factor & Exist & Prob \\
\hline $\begin{array}{l}\text { Wildlife } \\
\text { Threater } \\
\text { Threater } \\
\text { Federal } \\
\text { Federal } \\
\text { Federal } \\
\text { Federal } \\
\text { Federal } \\
\text { Federal } \\
\text { Federal }\end{array}$ & $\begin{array}{l}\text { e Value } \\
\text { ned/Endangered } \\
\text { ned/Endangered } \\
\text { Land Code } 103 \\
\text { Land Code } 104 \\
\text { Land Code } 105 \\
\text { Land Code } 106 \\
\text { Land Code } 107 \\
\text { Land Code } 108 \\
\text { Land Code } 198\end{array}$ & $\begin{array}{l}\text { Fish } \\
\text { wildifife }\end{array}$ & $\begin{array}{l}0.90 \\
0.90 \\
0.90 \\
0.90 \\
0.90 \\
0.90 \\
0.90 \\
0.90 \\
0.90 \\
0.90\end{array}$ \\
\hline
\end{tabular}

0 
RE SOURCE D A T A B A S E L I S T I N G

DATE: $10 / 05 / 93$

PAGE NO: 43

FERC

Number

Plant Name

stream

state

AR012 GUION

WHITE $R$

Name

AR

River Basin

IZARD

LOWER WHITE RIVER BASIN

Class Owner Name

NHPS/COE

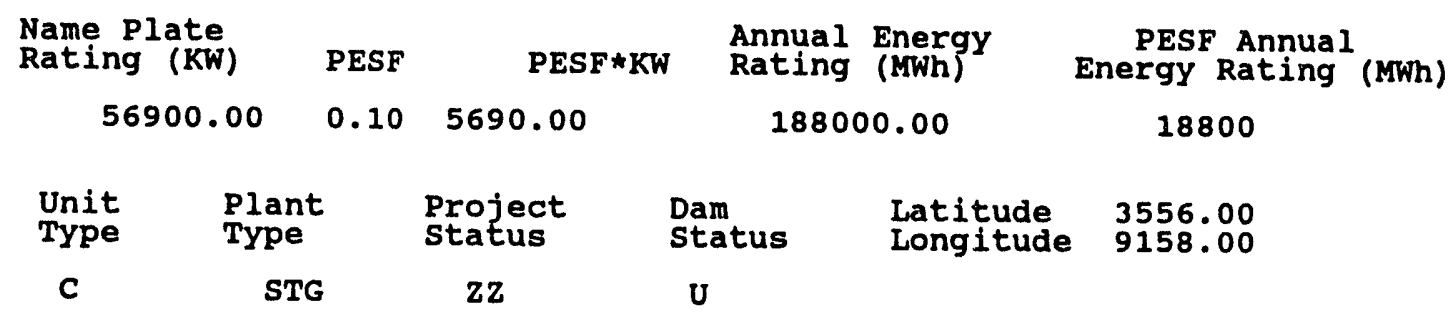

Factor

Exist Prob

Factor

Exist Prob

Wild/Scenic Protection

wild/scenic Tributary or

Upstream/Downstream

wild/Scenic Location

Cultural value

Fish Presence Value

Geologic Value

Historis Value

other Value

Recreation value

Scenic Value

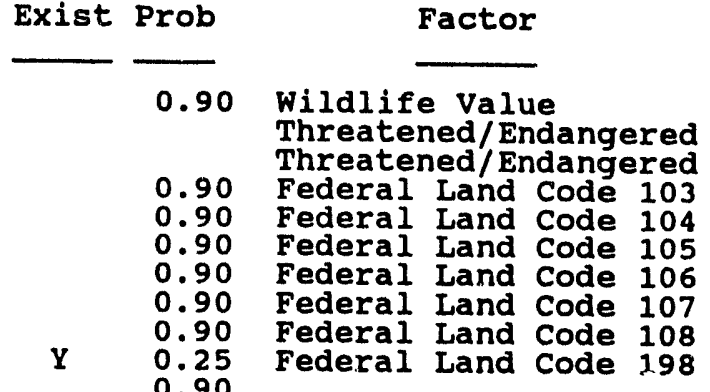

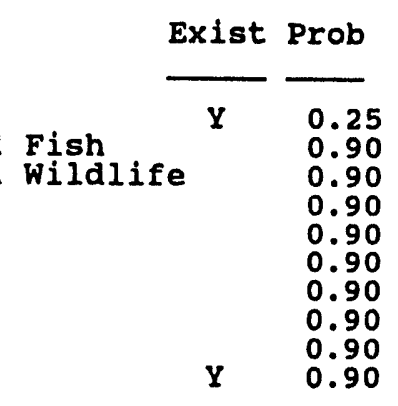


RE SOUR C E D A T A B A S E L I S T I N G

DATE : $10 / 05 / 93$

PAGE NO: 44

FERC

Number Plant Name

stream

State

AR013 BOSWELL

WHITE $\mathbf{R}$

AR

County Name

River Basin

IZARD

LOWER WHITE RIVER BASIN

Class Owner Name

NHPS/COE

\begin{tabular}{|c|c|c|c|c|}
\hline $\begin{array}{l}\text { Name Plate } \\
\text { Rating (KW) }\end{array}$ & PESF & $P E S F \star K W$ & $\begin{array}{l}\text { Annual Energy } \\
\text { Rating (MWh) }\end{array}$ & $\begin{array}{l}\text { PESF Annual } \\
\text { Energy Rating (MWh) }\end{array}$ \\
\hline 50300.00 & 0.50 & 25150.00 & 169100.00 & 84550 \\
\hline
\end{tabular}

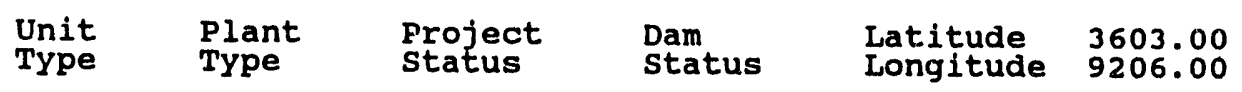

C

STG

ZZ

U

Factor

Exist Prob

Factor

Exist Prob

wild/Scenic Protection

wild/Scenic Tributary or Upstream/Downstream wild/Scenic Location Cultural value

Fish Presence Value

Geologic value

Historic Value

Other Value

Recreation Value

Scenic Value

0.90
0.90
0.90
0.90
0.90
0.90
0.90
0.90
0.90

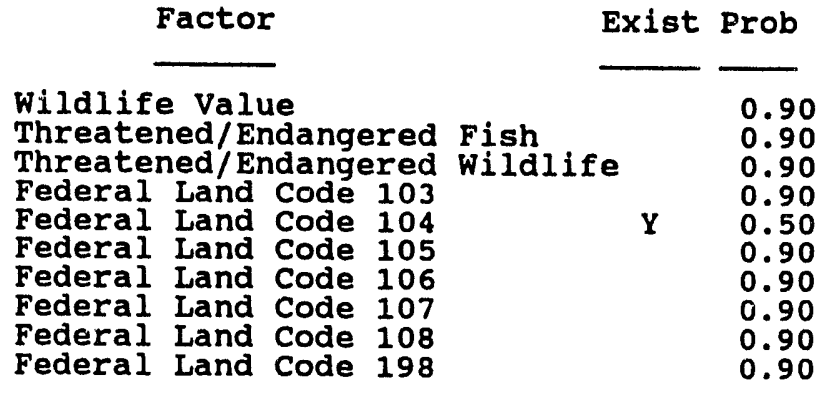


DATE: $10 / 05 / 93$

PAGE NO: 45

\section{FERC}

Number

Plant Name

stream

State

AR0 14

DEGRAY $3 /$

CADDO $\mathbf{R}$

Name

County Name

River Basin

CLARK

OUACHITA-BLACK RIVER BASIN

$\begin{array}{cc}\text { Class } & \text { Owner Name } \\ \text { F } & \text { CORPS OF ENGINEERS }\end{array}$

$\begin{array}{rcccc}\begin{array}{l}\text { Name Plate } \\ \text { Rating }(\mathrm{KW})\end{array} & \text { PESF } & \text { PESF*KW } & \begin{array}{l}\text { Annual Energy } \\ \text { Rating }\end{array} & \begin{array}{c}\text { PESF Annual } \\ \text { (MWh }\end{array} \\ 0.00 & 0.90 & 0.00 & 0.00 & 0\end{array}$

$\begin{array}{cccccc}\begin{array}{l}\text { Unit } \\ \text { Type }\end{array} & \begin{array}{l}\text { Plant } \\ \text { Type }\end{array} & \begin{array}{l}\text { Project } \\ \text { Status }\end{array} & \begin{array}{l}\text { Dam } \\ \text { Status }\end{array} & \begin{array}{l}\text { Latitude } \\ \text { Longitude }\end{array} & \begin{array}{l}3403.00 \\ 9307.00\end{array} \\ \text { C } & \text { CMB } & \text { FA } & w & \end{array}$

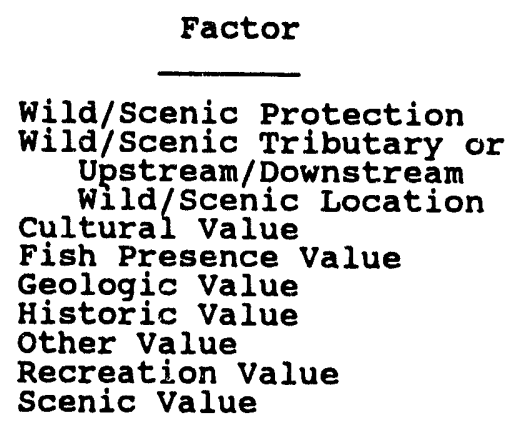

Exist Prob

Factor

Exist Prob

0.90

Wildife Value

Threatened/Endangered Fish

Threatened/Endangered Wildlife

0.90

0.90

0.90

0.90

0.90

Federal Land Code 103

Federal Land Code 104

Federal Land Code 105

Federal Land Code 106

Federal Land Code 107

0.90 Federal Land Code 108

0.90

Federal Land Code 198

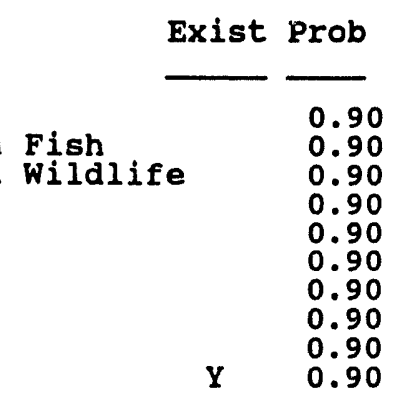



R E S O U R C E
D A T A B A S $\mathbf{E}$
L I S T I N G

DATE: $10 / 05 / 93$

PAGE NO: 46

FERC

Number

Plant Name

stream

state

ChASTAIN

WHITE R

Name

AR015

County Name

River Basin

BAXTER

LOWER WHITE RIVER BASIN

Class

Owner Name

NHPS/COE

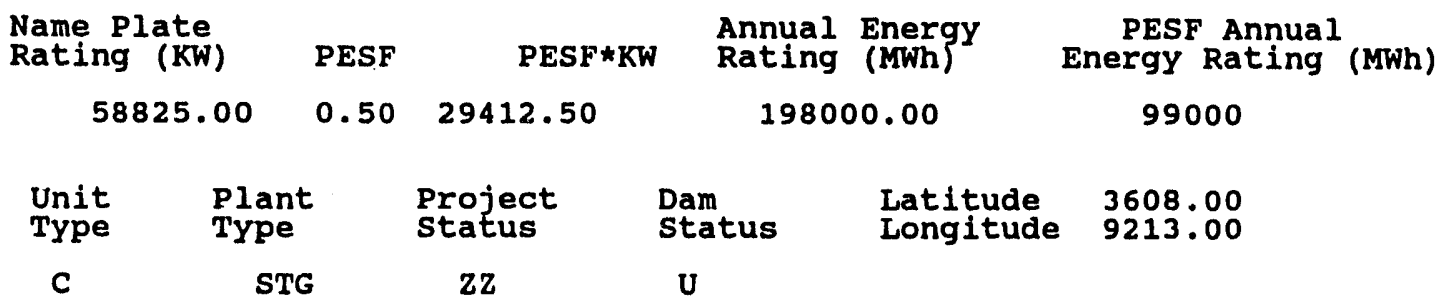

Factor

Exist Prob

Factor

Exist Prob

Wild/Scenic Protection

wild/Scenic Tributary or

Upstream/Downstream

Wild/Scenic Location

0.90
0.90
0.90
0.90
0.90
0.90
0.90
0.90
0.90

\begin{tabular}{|c|c|c|c|}
\hline & Factor & Exist & Prob \\
\hline $\begin{array}{l}\text { Wildlife } \\
\text { Threater } \\
\text { Threater } \\
\text { Federal } \\
\text { Federal } \\
\text { Federal } \\
\text { Federal } \\
\text { Federal } \\
\text { Federal } \\
\text { Federal }\end{array}$ & $\begin{array}{l}\text { e Value } \\
\text { ned/Endangered } \\
\text { ned/Endangered } \\
\text { Land Code } 103 \\
\text { Land Code } 104 \\
\text { Land Code } 105 \\
\text { Land Code } 106 \\
\text { Land Code } 107 \\
\text { Land Code } 108 \\
\text { Land Code } 198\end{array}$ & $\begin{array}{l}\text { Fish } \\
\text { Wildlife }\end{array}$ & $\begin{array}{l}0.90 \\
0.90 \\
0.90 \\
0.90 \\
0.50 \\
0.90 \\
0.90 \\
0.90 \\
0.90 \\
0.90\end{array}$ \\
\hline
\end{tabular}

Fish Presence Value

Geologic Value

Historic Value

other Value

Recreation Value

Scenic Value

0.90
0.90

0.90

. 90

0.50

.90 

R E S O U R C E
D A T A B A S E
I I S T I N G

DATE: $10 / 05 / 93$

PAGE NO: 47

FERC

Number

AR016

OZARK

Plant Name

County Name

FRANKLIN

\author{
stream \\ ARKANSAS $\mathbf{R}$ \\ Name \\ AR \\ River Basin \\ LOWER ARKANSAS RIVER BASIN
}

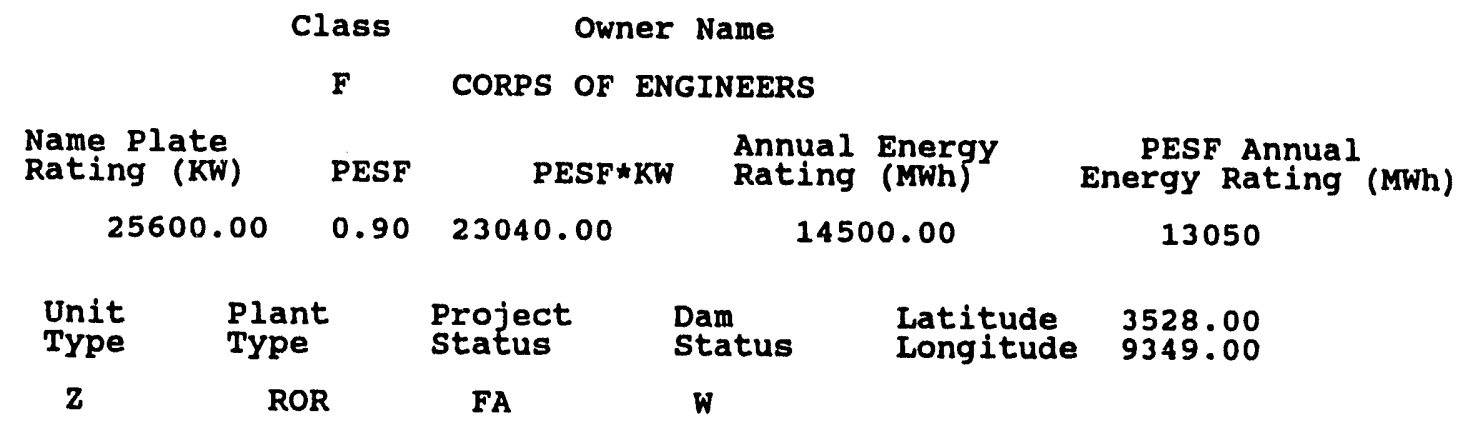

Factor

Exist Prob

Factor

Exist Prob

Wild/Scenic Protection

Wild/Scenic Tributary or Upstream/Downstream wild/Scenic Location

0.90
0.90
0.90
0.90
0.90
0.90
0.90
0.90
0.90

\begin{tabular}{lrl}
\multicolumn{1}{c}{ Factor } & Exist & Prob \\
& & \\
Wildlife Value & & 0.90 \\
Threatened/Endangered Fish & 0.90 \\
Threatened/Endangered Wildlife & 0.90 \\
Federal Land Code 103 & 0.90 \\
Federal Land Code 104 & 0.90 \\
Federal Land Code 105 & 0.90 \\
Federal Land Code 106 & 0.90 \\
Federal Land Code 107 & 0.90 \\
Federal Land Code 108 & & 0.90 \\
Federal Land Code 198 & & 0.90
\end{tabular}

Fish Presence Value

Geologic Value

Historic Value

other Value

Recreation Value

Scenic Value

0.90

0.90

.90

0

0.90

0.90 
RESOURCE DATABASE L I S T ING

DATE: $10 / 05 / 93$

PAGE NO: 48

FERC

Number

Plant Name

stream

State

AR017 BENTON

SALINE R

AR

County Name

SALINE
River Basin

OUACHITA-BLACK RIVER BASIN

Class Owner Name

-LMRCS

\begin{tabular}{|c|c|c|c|c|c|c|c|c|}
\hline \multirow{2}{*}{\multicolumn{2}{|c|}{$\begin{array}{l}\text { Name Plate } \\
\text { Rating (KW) } \\
25000.00\end{array}$}} & PESF & \multicolumn{2}{|c|}{$P E S F \star K W$} & \multicolumn{2}{|c|}{$\begin{array}{ll}\text { Annual Energy } \\
\text { Rating (MWh) }\end{array}$} & \multicolumn{2}{|c|}{$\begin{array}{r}\text { PESF An } \\
\text { Energy Rat }\end{array}$} \\
\hline & & 0.10 & 2500.00 & & \multicolumn{2}{|c|}{50000.00} & & 5000 \\
\hline $\begin{array}{l}\text { Unit } \\
\text { Type }\end{array}$ & \multicolumn{2}{|c|}{$\begin{array}{l}\text { Plant } \\
\text { Type }\end{array}$} & $\begin{array}{l}\text { Project } \\
\text { status }\end{array}$ & & atus & \multicolumn{2}{|c|}{$\begin{array}{l}\text { Latitude } \\
\text { Longitude }\end{array}$} & $\begin{array}{l}0.00 \\
0.00\end{array}$ \\
\hline C & \multicolumn{2}{|c|}{ STG } & $\mathbf{z z}$ & & & & & \\
\hline
\end{tabular}

Factor

Exist Prob

Factor

Exist Prob

wild/Scenic Protection

Wild/Scenic Tributary or

Upstream/Downstream

wild/Scenic Location

Cultural value

Fish Presence Value

Geologic Value

Historic Value

other Value

Recreation Value

Scenic Value

P

$\begin{array}{cc} & \\ & 0.90 \\ & \\ & 0.90 \\ Y & 0.90 \\ Y & 0.25 \\ Y & 0.90 \\ Y & 0.50 \\ Y & 0.90 \\ Y & 0.25 \\ & 0.50\end{array}$

Factor
Wildlife Value
Threatened/Endangered Fish
Threatened/Endangered Wildlife
Federal Land Code 103
Federal Land Code 104
Federal Land Code 105
Federal Land Code 106
Federal Land Code 107
Federal Land Code 108
Federal Land Code 198

0.25

0.90

0.90

0.90

0.90

0.90

0.90

0.90

0.90

0.90 
R E S O U R C E

DATE: $10 / 05 / 93$

FERC

Number

Plant Name

AR018

DIVERSION DAM

County Name

HEMPSTEAD
D A T A B A S E

I I S T I N G

PAGE NO: 49

stream

RED $\mathbf{R}$
River Basin

LOWER RED RIVER BASIN

Class Owner Name

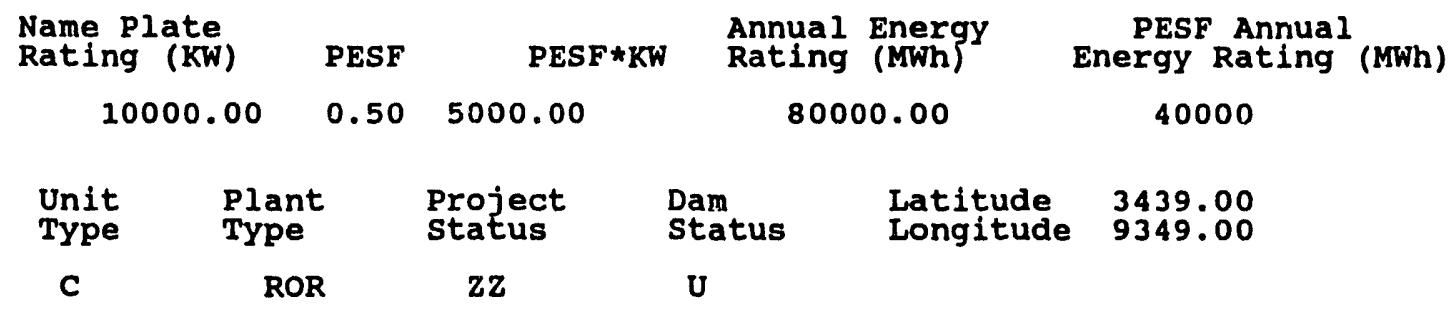

Factor

Exist Prob

Factor

Exist Prob

Wild/Scenic Protection

wild/Scenic Tributary or Upstream/Downstream

wild/Scenic Location

0.90
0.90
0.90
0.90
0.90
0.90
0.90
0.90

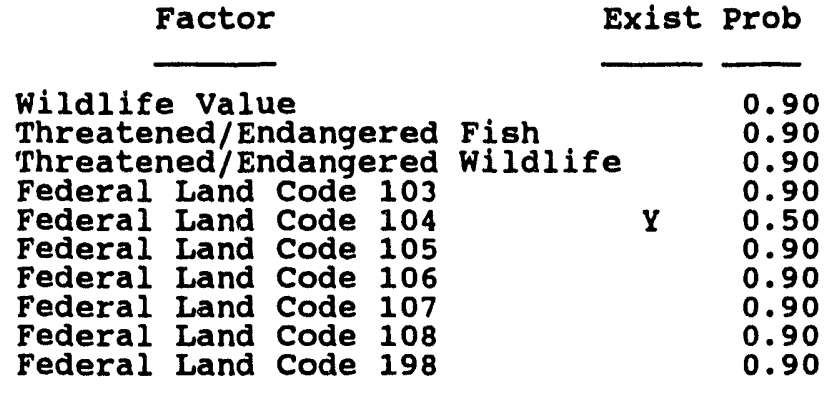

Fish Presence Value

Geologíc Value

Historic Value

other Value

Recreation Value

0.90

Federal Land Code 198

AR

State

Name

AR

Scenic Value 
RESOUR CE D A T A B A S E L I S T I N G

DATE: $10 / 05 / 93$

PAGE NO: 50

FERC.

Number

Plant Name

stream

state

AR019 HILTON DAM 3

SPRING $R$

Name

County Name FULTON

River Basin

LOWER WHITE RIVER BASIN

Class Owner Name

P AR-MO PWR CO

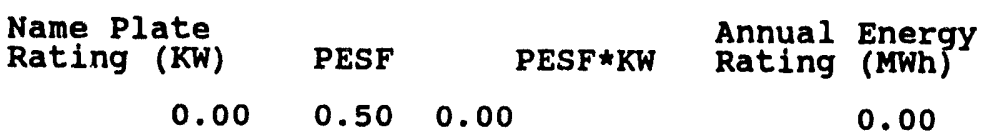

PESF Annual

Energy Rating (MWh)

0.00

$0.50 \quad 0.00$
Dam Latitude
Longitude
status
พอ

3629.00

Type

Type

Project

status

9132.00

$\mathbf{z}$

ROR

ZZ

Factor

Exist Prob

Factor

Wild/Scenic Protection

wild/Scenic Tributary or Upstream/Downstream wild/Scenic Location Cultural value

Fish Presence Value

Geologic Value

Historic Value

other Value

Recreation Value

Scenic Value

\begin{tabular}{cc}
\hline & 0.90 \\
& \\
& \\
& 0.90 \\
$Y$ & 0.90 \\
$Y$ & 0.75 \\
& 0.90 \\
& 0.90 \\
$Y$ & 0.90 \\
$Y$ & 0.75 \\
& 0.90
\end{tabular}

Wildlife Value

Threatened/Endangered Fish

Threatened/Endangered Wildlife

Federal Land Code 103

Federal Land Code 104

Federal Land Code 105

Federal Land Code 106

Federal Land Code 107

Federal Land Code 108

Federal Land Code 198
Exist Prob

AR

0

\begin{tabular}{ll}
\hline \multicolumn{1}{c}{$Y$} & \\
& 0.75 \\
& 0.90 \\
& 0.90 \\
& 0.90 \\
& 0.90 \\
& 0.90 \\
& 0.90 \\
& 0.90 \\
& 0.90 \\
$Y$ & 0.90
\end{tabular}


R E S O U R E

DATE: $10 / 05 / 93$

FERC

Number

AR020

FULTON L\&D

County Name

HEMPSTEAD
D A T A B A S E

L I $\mathbf{S}$ T I $\mathbf{N}$ G

PAGE NO: 51

stream

state

Name

RED $\mathbf{R}$

AR

River Basin

LOWER RED RIVER BASIN

\begin{tabular}{|c|c|c|c|c|c|c|}
\hline \multicolumn{3}{|c|}{ Class } & \multicolumn{2}{|c|}{ Owner Name } & & \multirow[b]{3}{*}{$\begin{array}{l}\text { PESF Annual } \\
\text { Energy Rating (MWh) }\end{array}$} \\
\hline \multirow{2}{*}{\multicolumn{2}{|c|}{$\begin{array}{l}\text { Name Plate } \\
\text { Rating (KW) }\end{array}$}} & $\mathbf{F}$ & CORPS OF EI & SNGINEERS & & \\
\hline & & PESF & PESF *KI & $\begin{array}{l}\text { Annual } \\
\text { Rating }\end{array}$ & $\begin{array}{l}\text { Energy } \\
(\text { MWh })\end{array}$ & \\
\hline \multicolumn{2}{|c|}{17989.00} & 0.90 & 16190.10 & \multicolumn{2}{|c|}{98137.00} & 88323.30 \\
\hline $\begin{array}{l}\text { Unit } \\
\text { Type }\end{array}$ & $\begin{array}{l}\text { Pl } \\
\text { TYi }\end{array}$ & & $\begin{array}{l}\text { Project } \\
\text { status }\end{array}$ & $\begin{array}{l}\text { Dam } \\
\text { status }\end{array}$ & $\begin{array}{l}\text { Latitude } \\
\text { Longitude }\end{array}$ & $\begin{array}{l}3336.00 \\
9349.00\end{array}$ \\
\hline C & & & $\mathbf{z z}$ & $\mathbf{U}$ & & \\
\hline
\end{tabular}

Factor

Exist Prob

Factor

Exist Prob

Wild/Scenic protection

wild/Scenic Tributary or

Upstream/Downstream Wild/Scenic Location

Cultural value

Fish Presence Value

Geologic Value

D.90
-9.90
0.90
0.90
0.90
0.90
0.90
0.90
0.90
0.90

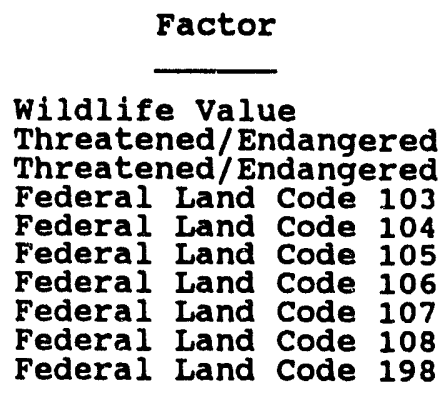

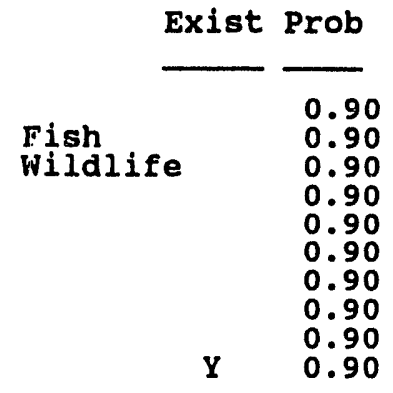

Recreation Value

Scenic Value 

R E S S O U R R C E
D A T A B A E
I I $\mathbf{S}$ T I $N G$

DATE: $10 / 05 / 93$

PAGE NO: 52

FERC

Number

Plant Name

stream

state

AR02 1

HARTLEY

COSSATOT $\mathbf{R}$

AR

County Name

River Basin

HOWARD

LOWER RED RIVER BASIN

$\begin{array}{cc}\text { Class } & \text { Owner Name } \\ \text { F } & \text { CORPS OF ENGINEERS }\end{array}$

Name Plate

Rating (KW)

PESF

PESF $\star K W$

Annual Energy

Rating (MWh)

PESF Annual

14200.00

$0.10 \quad 1420.00$

16600.00

Energy Rating (MWh)

Unit Plant

Type Type

Project

status

Dam

status

Latitude

C

STG

ZZ

U

Factor
Wild/Scenic Protection
wild/Scenic Tributary or
Upstream/Downstream
Wild/Scenic Location
Cultural Value
Fish Presence Value
Geologic Value
Historic Value
Other Value
Recreation Value
Scenic Value

Exist Prob

Factor

Exist Prob

\begin{tabular}{cc}
\cline { 2 - 2 } & 0.90 \\
& \\
& 0.90 \\
& 0.90 \\
$Y$ & 0.25 \\
$Y$ & 0.50 \\
& 0.90 \\
$Y$ & 0.90 \\
$Y$ & 0.25 \\
$Y$ & 0.50
\end{tabular}

Wildife Value Threatened/Endangered Fish Threatened/Endangered Wildife

Y 0.25

Federal Land Code 103

Federal Land Code 104

Federal Land Code 105

Federal Land Code 106

Federal Land Code 107

Federal Land Code 108

Federal Land Code 198

3413.00

9414.00

0.25
0.50

0.90

0.90

0.90

0.90

0.90

0.90

Y $\quad 0.90$ 
RESOURCE DA TABA S E L I S T I N G

DATE: $10 / 05 / 93$

PAGE NO: 53

FERC

Number

Plant Name

stream

State

AR022 HARDY

SPRING $\mathbf{R}$

AR

County Name

SHARP

River Basin

LOWER WHITE RIVER BASIN

Class Owner Name

\begin{tabular}{|c|c|c|c|c|c|c|}
\hline \multirow{2}{*}{\multicolumn{2}{|c|}{$\begin{array}{l}\text { Name Plate } \\
\text { Rating (KW) } \\
\qquad 52000.00\end{array}$}} & \multirow{2}{*}{$\begin{array}{l}\text { PESF } \\
0.10\end{array}$} & PESF $\star K W$ & \multicolumn{2}{|c|}{$\begin{array}{l}\text { Annual Energy } \\
\text { Rating (MWh) }\end{array}$} & $\begin{array}{l}\text { PESF Annual } \\
\text { Energy Rating (MWh) }\end{array}$ \\
\hline & & & 5200.00 & \multicolumn{2}{|c|}{79000.00} & 7900 \\
\hline $\begin{array}{l}\text { Unit } \\
\text { Type }\end{array}$ & $\begin{array}{l}\text { Pla } \\
\text { TYI }\end{array}$ & & $\begin{array}{l}\text { Project } \\
\text { Status }\end{array}$ & $\begin{array}{l}\text { Dam } \\
\text { status }\end{array}$ & $\begin{array}{l}\text { Latitude } \\
\text { Longitude }\end{array}$ & $\begin{array}{l}3620.00 \\
9130.00\end{array}$ \\
\hline C & & & $\mathbf{z z}$ & $\mathbf{U}$ & & \\
\hline
\end{tabular}

Factor

Exist Prob

Factor

Exist Prob

Wild/Scenic Protection

wild/Scenic Tributary or

Upstream/Downstream

wild/Scenic Location

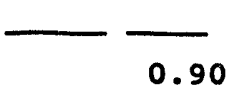

0.90

wildlife Value

Threatened/Endangered Fish

0.25
0.90
0.90
0.90
0.90
0.90
0.90
0.90
0.90

0.90

Fish Presence Value

Geologic value

Threatened/Endangered

0.90 Federal Land Code 103

0.90 Federal Land Code 104

$Y \quad 0.25$ Federal Land Code 105

0.50 Federal Land Code 106

0.90 Federal Land Code 107

other Value

0.90 Federal Land Code 108

Recreation Value

0.25

Federal Land Code 198

$\begin{array}{lll} & & \\ \text { Fish } & Y & 0.25 \\ \text { Wildife } & 0.90 \\ & 0.90 \\ & 0.90 \\ & 0.90 \\ & & 0.90 \\ & 0.90 \\ & 0.90 \\ & 0.90 \\ & & 0.90\end{array}$



RESOU R C E
D A T A B A S E
I I S I N G

DATE: $10 / 05 / 93$

PAGE NO: 54

FERC

Number

AR02 3
Plant Name

WOLF BAYOU REREGULATOR

County Name

INDEPENDENCE

\section{stream}

WHITE $R$

River Basin

\section{Class}

Owner Name

NHPS/COE

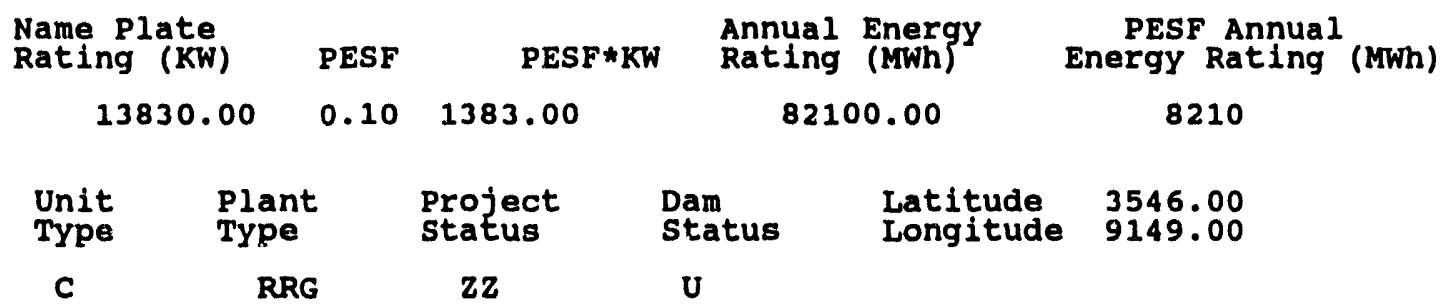

Factor

Exist Prob

Factor

Exist Prob

Wild/Scenic Protection

Wild/Scenic Tributary or

Upstream/Downstream wild/Scenic Location Cultural value

Fish Presence Value

Geologic Value

Historic Value

Other Value

Recreation Value

Scenic Value

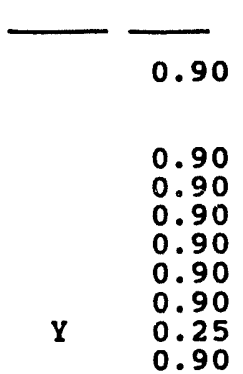

\begin{tabular}{|c|c|c|c|c|}
\hline & Factor & & Exist & Prob \\
\hline $\begin{array}{l}\text { Wildilf } \\
\text { Threate } \\
\text { Threate } \\
\text { Federal } \\
\text { Federal } \\
\text { Federal } \\
\text { Federal } \\
\text { Federal } \\
\text { Federal } \\
\text { Federal }\end{array}$ & $\begin{array}{l}\text { V Value } \\
\text { ned/Endangered } \\
\text { ned/Endangered } \\
\text { Land Code } 103 \\
\text { Land Code } 104 \\
\text { Land Code } 105 \\
\text { Land Code } 106 \\
\text { Land Code } 107 \\
\text { Land Code } 108 \\
\text { Land Code } 198\end{array}$ & $\begin{array}{l}\text { Fish } \\
\text { wildlife }\end{array}$ & $e^{Y}$ & $\begin{array}{l}0.25 \\
0.90 \\
0.90 \\
0.90 \\
0.90 \\
0.90 \\
0.90 \\
0.90 \\
0.90 \\
0.90\end{array}$ \\
\hline
\end{tabular}


RESOURCE DATABASE L I S T ING

DATE : $10 / 05 / 93$

PAGE NO: 55

FERC

Number

ARO2 4
Plant Name

WOLF BAYOU

County Name

INDEPENDENCE

\section{stream}

WHITE $\mathbf{R}$

River Basin

LOWER WHITE RIVER BASIN

Class

Owner Name

NHPS/COE

\begin{tabular}{|c|c|c|c|c|c|c|}
\hline \multicolumn{2}{|c|}{$\begin{array}{l}\text { Name Plate } \\
\text { Rating (KW) }\end{array}$} & PESF & $\mathrm{PESF} \star \mathrm{KW}$ & $\begin{array}{l}\text { Ann } \\
\text { Rat }\end{array}$ & $\begin{array}{l}\text { Energy } \\
\text { (MWh) }\end{array}$ & $\begin{array}{l}\text { PESF Annual } \\
\text { Energy Rating (MWh) }\end{array}$ \\
\hline \multicolumn{2}{|c|}{125000.00} & 0.10 & 12500.00 & \multicolumn{2}{|c|}{347100.00} & 34710 \\
\hline $\begin{array}{l}\text { Unit } \\
\text { Type }\end{array}$ & $\begin{array}{l}\text { Pla } \\
\text { Typ }\end{array}$ & & $\begin{array}{l}\text { Project } \\
\text { status }\end{array}$ & $\begin{array}{l}\text { Dam } \\
\text { status }\end{array}$ & $\begin{array}{l}\text { Latitude } \\
\text { Longitude }\end{array}$ & $\begin{array}{l}3549.00 \\
9152.00\end{array}$ \\
\hline C & & & $\mathbf{z z}$ & $\mathbf{U}$ & & \\
\hline
\end{tabular}

Factor

Exist Prob

Factor

Exist Prob

wild/scenic Protection

wild/scenic Tributary or Upstream/Downstream wild/Scenic Location

\begin{tabular}{l}
0.90 \\
\hline
\end{tabular}

\begin{tabular}{|c|c|c|c|}
\hline & Factor & Exist & Prob \\
\hline $\begin{array}{l}\text { Wildlife } \\
\text { Threaten } \\
\text { Threaten } \\
\text { Federal } \\
\text { Federal } \\
\text { Federal } \\
\text { Federal } \\
\text { Federal } \\
\text { Federal } \\
\text { Federal }\end{array}$ & $\begin{array}{l}\text { e Value } \\
\text { ned/Endangered } \\
\text { ned/Endangered } \\
\text { Land Code } 103 \\
\text { Land Code } 104 \\
\text { Land Code } 105 \\
\text { Land Code } 106 \\
\text { Land Code } 107 \\
\text { Land Code } 108 \\
\text { Land Code } 198\end{array}$ & $\begin{array}{l}\text { Fish } \\
\text { Wildife } \\
Y\end{array}$ & $\begin{array}{l}0.25 \\
0.90 \\
0.90 \\
0.90 \\
0.90 \\
0.90 \\
0.90 \\
0.90 \\
0.90 \\
0.90\end{array}$ \\
\hline
\end{tabular}

Cultural value

Fish Presence Value

Geologic Value

Historic Value

other Value

Recreation Value

Scenic Value

0.25

.90

0.90

0.90

0.90

0.90

Federal Land Code 108

.90 
RESOUR C E DA TABA S E L I S T I N G

DATE: $10 / 05 / 93$

FERC

Number

Plant Name

PAGE NO: 56

AR025

GREERS FERRY

County Name

CLEBURNE

State

LITTLE RED R

Name

AR

River Basin

LOWER WHITE RIVER BASIN

$\begin{array}{cc}\text { Class } & \text { Owner Name } \\ \text { F } & \text { CORPS OF ENGINEERS }\end{array}$

\begin{tabular}{|c|c|c|c|c|c|}
\hline $\begin{array}{l}\text { Name plate } \\
\text { Rating (KW) }\end{array}$ & PESF & PESF $\star K W$ & $\begin{array}{l}\text { Annual } \\
\text { Rating }\end{array}$ & $\begin{array}{l}\text { Energy } \\
\text { (MWh) }\end{array}$ & $\begin{array}{l}\text { DESF Annual } \\
\text { Energy Rating (MWh) }\end{array}$ \\
\hline 0.00 & 0.50 & 0.00 & & 0.00 & 0 \\
\hline
\end{tabular}

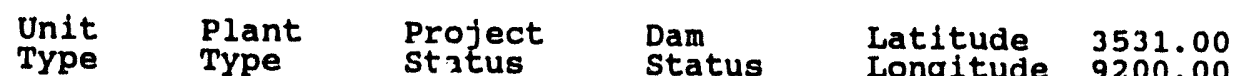

z STG FA

Factor

Exist Prob

Factor

Exist Prob

Wild/Scenic Protection

Wild/Scenic Tributary or

Upstream/ Downstream

wild/Scenic Location

Cultural value

Fish Presence Value

Geologíc Value

Historic Value

other Value

Recreation value

Scenic Value

\begin{tabular}{cc} 
Exist & Prob \\
\cline { 1 - 1 } & 0.90 \\
& \\
& \\
& 0.90 \\
$Y$ & 0.90 \\
& 0.75 \\
& 0.90 \\
$Y$ & 0.90 \\
$Y$ & 0.90 \\
$Y$ & 0.75 \\
& 0.90
\end{tabular}

\begin{tabular}{l} 
Factor \\
\cline { 2 - 2 } Wildife Value \\
Threatened/Endangered \\
Threatened/Endangered \\
Federal Land Code 103 \\
Federal Land Code 104 \\
Federal Land Code 105 \\
Federal Land Code 106 \\
Federal Land Code 107 \\
Federal Land Code 108 \\
Federal Land Code 198
\end{tabular}

\begin{tabular}{ll}
\multicolumn{1}{c}{ Exist } & Prob \\
\cline { 2 - 2 } Fish & 0.90 \\
Wildife & 0.90 \\
& 0.90 \\
& 0.90 \\
& 0.90 \\
& 0.90 \\
& 0.90 \\
& 0.90 \\
& 0.90 \\
& 0.90
\end{tabular}


R E S O U R C E

DATE: $10 / 05 / 93$

FERC

Number

Plant Name

AR026 QUARRY

County Name

CLEBURNE
D A $\mathbf{A}$ A B A $\mathbf{S} \mathbf{E}$

L $\mathbf{S}$ T I N G

PAGE NO: 57

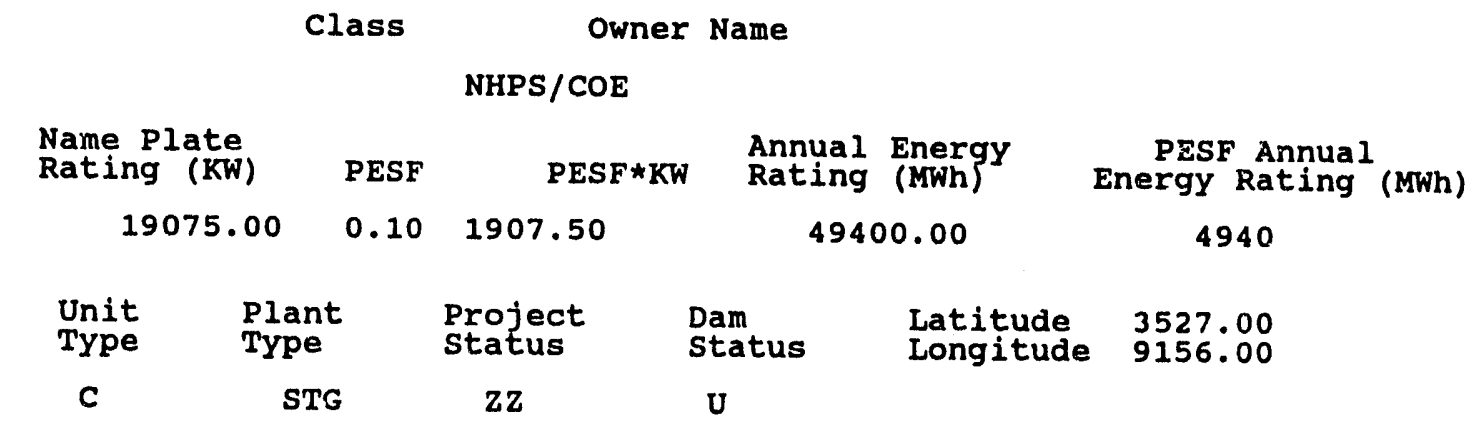

Factor

Exist Prob

Wild/Scenic Protection

Wild/Scenic Tributary or Upstream/Downstream wild/scenic Location Cultural value

Fish Presence Value

Geologic Value

Historic Value

Other Value

Recreation Value

Scenic value

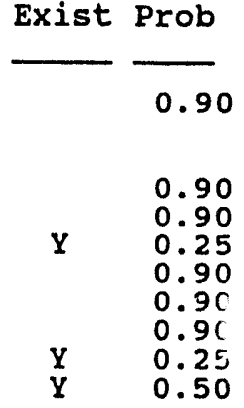

Factor

Exist Prob

Wildife Value

Threatened/Endangered Fish

Threatened/Endangered Wildlife

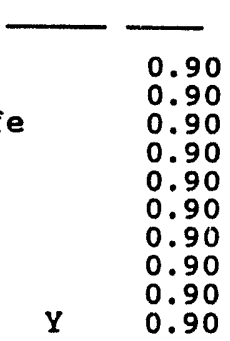


DATE: $10 / 05 / 93$

PAGE NO: 58

FERC

Number

Plant Name

AR027

BULL SHOALS 3/

County Name

BAXTER

$\begin{array}{cc}\text { stream } & \begin{array}{c}\text { State } \\ \text { Name }\end{array} \\ \text { WHITE } R & A R\end{array}$

River Basin

UPPER WHITE RIVER BASIN

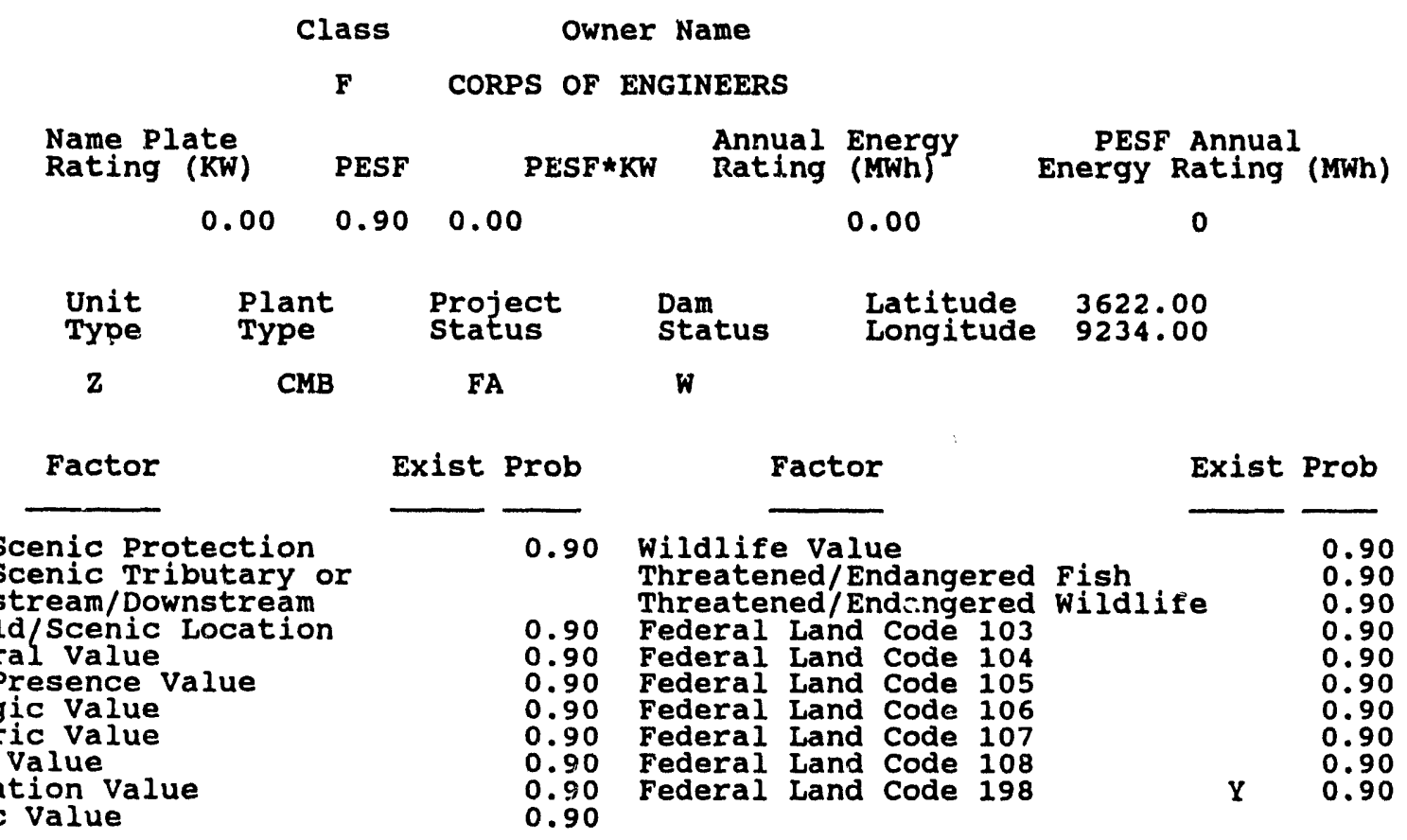


RE SOU R C E D A T A B A S E I I S T I N G

DATE: $10 / 05 / 93$

PAGE NO: 59

FERC

Number

AR02 8

BELL FOLEY

County Name

SHARP

Plant Name

STRAWBERRY $R$

River Basin

LOWER WHITE RIVER BASIN

Class Owner Name

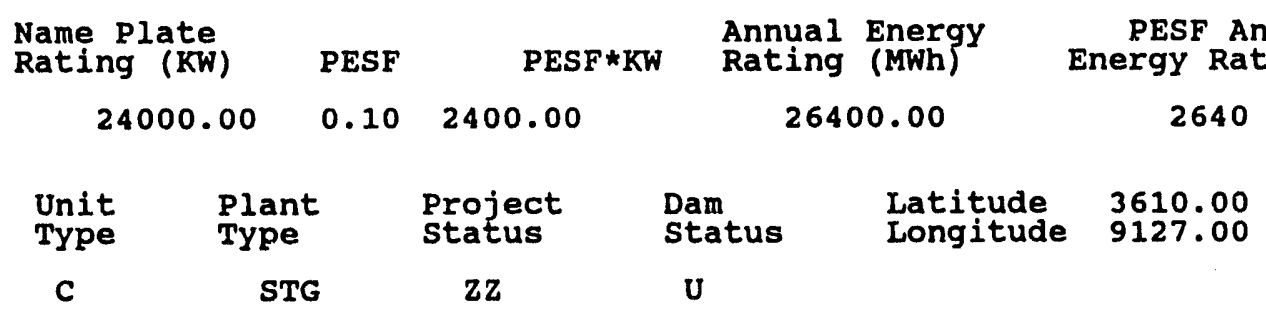

Factor

\begin{tabular}{cc} 
Exist & Prob \\
\cline { 1 - 2 } & \multicolumn{1}{c}{0.90} \\
& \\
& 0.90 \\
& 0.90 \\
$Y$ & 0.25 \\
$Y$ & 0.50 \\
& 0.90 \\
$Y$ & 0.90 \\
$Y$ & 0.25 \\
$Y$ & 0.50
\end{tabular}

Factor

Exist Prob

wild/Scenic Protection

wild/Scenic Tributary or Upstream/Downstream Wild/Scenic Location Cultural value

Fish Presence Value

Geologic Value

Historic Value

other value

Recreation value

scenic Value

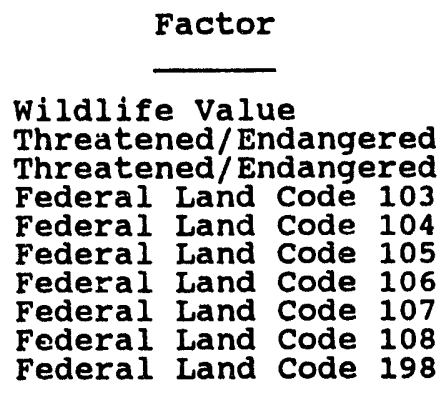

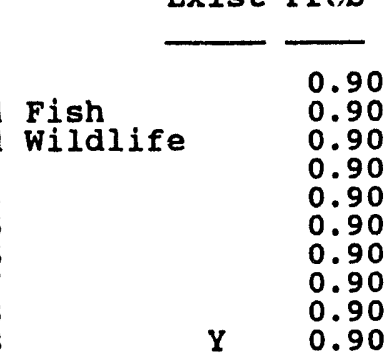


RE SOU R C E D A T A B A S E I I S T I N G

DATE : $10 / 05 / 93$

FERC

Number

Plant Name

AR029 COTTER

County Name

BAXTER

Owner Name

NHPS/COE

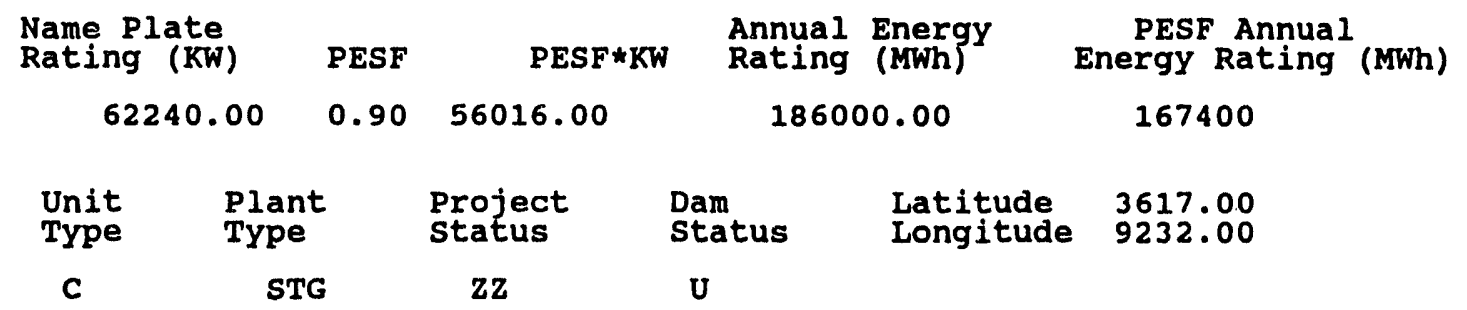

Factor

Exist Prob

Factor

Exist Prob

Wild/Scenic Protection

Wild/Scenic Tributary or Upstream/Downstream

wild/Scenic Location

Cultural value

Fish Presence Value

Geologic Value

Historic Value

other Value

Recreation Value

Scenic Value
PAGE NO: 60

State

Name

WHITE $R$

AR

River Basin

UPPER WHITE RIVER BASIN

\begin{tabular}{|c|c|c|c|c|c|}
\hline & Factor & & & Exist & Prob \\
\hline $\begin{array}{l}\text { Wildlif } \\
\text { Threate } \\
\text { Threate } \\
\text { Federal } \\
\text { Federal } \\
\text { Federal } \\
\text { Federal } \\
\text { Federal } \\
\text { Federal } \\
\text { Federal }\end{array}$ & $\begin{array}{l}\text { Ee Value } \\
\text { ened/End } \\
\text { ened/End } \\
\text { Land C } \\
\text { Land C } \\
\text { Land C } \\
\text { Land C } \\
\text { Land C } \\
\text { Land C } \\
\text { Land C }\end{array}$ & $\begin{array}{l}\text { e } \\
\text { idangered } \\
\text { idangered } \\
\text { Code } 103 \\
\text { Code } 104 \\
\text { Code } 105 \\
\text { Code } 106 \\
\text { Code } 107 \\
\text { Code } 103 \\
\text { Code } 198\end{array}$ & $\begin{array}{l}\text { Fish } \\
\text { wildife }\end{array}$ & e & $\begin{array}{l}0.90 \\
0.90 \\
0.90 \\
0.90 \\
0.90 \\
0.90 \\
0.90 \\
0.90 \\
0.90 \\
0.90\end{array}$ \\
\hline
\end{tabular}


R E S O R C E D A T A B A S E L I S T I N G

DATE: $10 / 05 / 93$

PAGE NO: 61

FERC

Number

Plant Name

AR030 NORFORK

County Name

BAXTER

River Basin

UPPER WHITE RIVER BASIN

$\begin{array}{cc}\text { Class } & \text { Owner Name } \\ \text { F } & \text { CORPS OF ENGINEERS }\end{array}$

Name Plate

Annual Energy

Rating (KW)

PESF

PESF*KW

Rating (MWh)

85000.00

0.9076500 .00

22000.00

PESF Annual

Energy Rating (MWh)

\begin{tabular}{|c|c|c|c|}
\hline $\begin{array}{l}\text { Unit } \\
\text { Type }\end{array}$ & $\begin{array}{l}\text { Plant } \\
\text { Type }\end{array}$ & $\begin{array}{l}\text { Project } \\
\text { status }\end{array}$ & $\begin{array}{l}\text { Dam } \\
\text { status }\end{array}$ \\
\hline
\end{tabular}

C

STG

FA

พ

Factor

Exist Prob

Wild/Scenic Protection

wild/Scenic Tributary or Upstream/Downstream wild/Scenic Location

Cultural value

Fish Presence Value

Geologic Value

Historic Value

other Value

Recreation Value

Scenic Value

0.90
0.90
0.90
0.90
0.90
0.90
0.90
0.90
0.90

Factor

Exist Prob

Wildlife Value

Threatened/Endangered Fish

Threatened/Endangered Wildlife

Federal Land Code 103

Federal Land Code 104

Federal Land Code 105

Federal Land Code 106

Federal Land Code 107

Federal Land Code 108

Federal Land Code 198

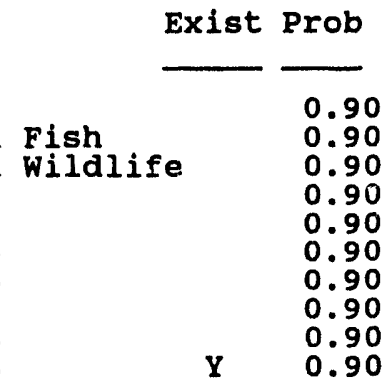



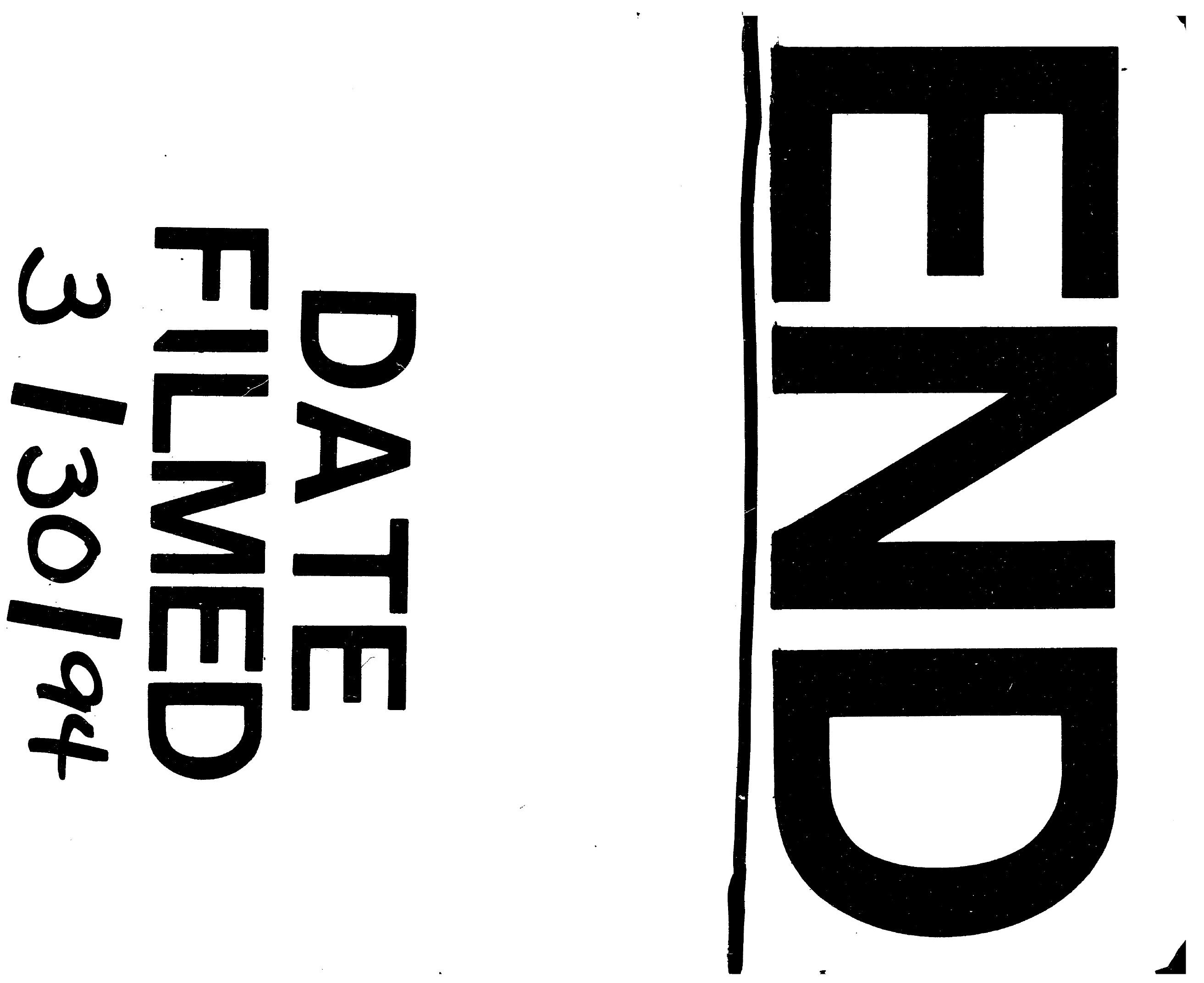
